\title{
Adaptive Non-Local Means using Weight Thresholding
}

Asif Khan, The University of Western Ontario

Supervisor: Dr. Mahmoud R. El-Sakka, The University of Western Ontario

A thesis submitted in partial fulfillment of the requirements for the Master of Science degree in Computer Science

(C) Asif Khan 2015

Follow this and additional works at: https://ir.lib.uwo.ca/etd

Part of the Computer Sciences Commons

\section{Recommended Citation}

Khan, Asif, "Adaptive Non-Local Means using Weight Thresholding" (2015). Electronic Thesis and Dissertation Repository. 3369.

https://ir.lib.uwo.ca/etd/3369

This Dissertation/Thesis is brought to you for free and open access by Scholarship@Western. It has been accepted for inclusion in Electronic Thesis and Dissertation Repository by an authorized administrator of Scholarship@Western. For more information, please contact wlswadmin@uwo.ca. 


\title{
ADAPTIVE NON-LOCAL MEANS USING WEIGHT THRESHOLDING (Thesis format: Monograph) \\ by
}

\begin{abstract}
Asif Khan
Graduate Program in Department of Computer Science

A thesis submitted in partial fulfillment

of the requirements for the degree of

Masters of Science
\end{abstract}

The School of Graduate and Postdoctoral Studies

The University of Western Ontario

London, Ontario, Canada

(c) Asif Khan 2015 


\begin{abstract}
Non-local means (NLM) is a popular image denoising scheme for reducing additive Gaussian noise. It uses a patch-based approach to find similar regions within a search neighborhood and estimate the denoised pixel based on the weighted average of all the pixels in the neighborhood. All the pixels are considered for averaging, irrespective of the value of their weights. This thesis proposes an improved variant of the original NLM scheme, called Weight Thresholded Non-Local Means (WTNLM), by thresholding the weights of the pixels within the search neighborhood, where the thresholded weights are used in the averaging step. The key parameters of the WTNLM are defined using learning-based models. In addition, the proposed method is used as a two-step approach for image denoising. At the first step, WTNLM is applied to generate a basic estimate of the denoised image. The second step applies WTNLM once more but with different smoothing strength. Experiments show that the denoising performance of the proposed method is better than that of the original NLM scheme, and its variants. It also outperforms the state-of-the-art image denoising scheme, BM3D, but only at low noise levels $(\sigma \leq 80)$.
\end{abstract}

Keywords: Image denoising, Additive Gaussian Noise, Non-local Means, Two-stage Nonlocal means, Spatial Domain Denoising 


\section{Acknowledgments}

I am expressing my heartiest gratitude to the Almighty to give me the ability to complete my thesis successfully. I would like to thank my thesis supervisor Dr. Mahmoud R. El-Sakka for his noteworthy and valuable direction, guidance, motivation and encouragement in the way of my progress. It was an absolute honor and privilege to work with such a modest and wise person like him. Moreover, I am also thankful to all of my professors from the University of Western Ontario for building my background to complete this task.

I am also grateful to my family for their continuous support and guidance. 


\section{Contents}

$\begin{array}{ll}\text { Abstract } & \text { ii }\end{array}$

Acknowledgments $\quad$ iii

List of Figures $\quad$ vii

List of Tables $\quad$ ix

1 Introduction $\quad 1$

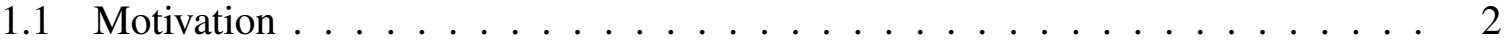

1.2 Thesis Contribution . . . . . . . . . . . . . . . . 2

1.3 Thesis Outline . . . . . . . . . . . . . . . . . . 3

2 Background $\quad 4$

$2.1 \quad$ Image Noise . . . . . . . . . . . . . . . . . . . 4

2.1.1 Additive Noise . . . . . . . . . . . . . . . . . . 4

White Gaussian Noise . . . . . . . . . . . . . . 5

Estimating additive white Gaussian noise . . . . . . . . . . . 6

2.1.2 Multiplicative Noise . . . . . . . . . . . . . 7

2.1.3 Salt \& Pepper Noise . . . . . . . . . . . . . . 7

2.2 Image Denoising . . . . . . . . . . . . . . 7

2.2.1 Spatial Domain Approach _............... . . 8

2.2.2 Frequency Domain Approach . . . . . . . . . . . . . 8 
2.3 Spatial Domain Methods _. . . . . . . . . . . . . . . . . . . . . 9

$2.3 .1 \quad$ Mean Filter . . . . . . . . . . . . . . . . . . . . . . 9

2.3.2 Median Filter . . . . . . . . . . . . . . . . . . . 9

2.3.3 Gaussian smoothing . . . . . . . . . . . . . . 10

2.3.4 Anisotropic Diffusion . . . . . . . . . . . . . . . 10

2.3.5 2-D Adaptive Wiener Filter . . . . . . . . . . . . . . . . . 11

2.4 Frequency Domain Methods _. . . . . . . . . . . . . . . . . 12

$2.4 .1 \quad$ Low-Pass Filter . . . . . . . . . . . . . . . . . . 12

2.4 .2 Wiener Filter . . . . . . . . . . . . . . . . . . 13

$2.4 .3 \quad$ BM3D . . . . . . . . . . . . . . . . . . . 14

2.4 .4 BM3D Extensions $\ldots \ldots \ldots \ldots \ldots \ldots \ldots$

BM3D Denoising using Shape-Adaptive Principal Component Analysis 16

BM3D Denoising using SSIM Optimized Wiener Filter . . . . . . . 16

2.5 Non-Local Means . . . . . . . . . . . . . . . . . . . . . . . . 17

2.6 Non-Local Means Variants $\ldots \ldots \ldots$

2.6.1 Principal Components of Non-Local Means . . . . . . . . . . 18

2.6.2 Improved Non-Local Means based on dimensionality reduction . . . . . 19

2.6.3 SSIM-based Non-Local Means . . . . . . . . . . . . . . . . . 20

2.6 .4 Iterative Non-Local Means . . . . . . . . . . . . . . . . 20

2.6.5 Non-Local Euclidean Median . . . . . . . . . . . . . . . . . 21

2.6.6 Two-stage Non-Local Means with adaptive smoothing parameters . . 21

2.7 Non-Local Means Applications . . . . . . . . . . . . . . . . . . . . 22

3 Methodology 23

3.1 Adaptive Non-Local Means using Weight Thresholding . . . . . . . . . . 23

3.2 Parameter Selection . . . . . . . . . . . . . . . . . 25

3.2.1 Cut-off Weight for Thresholding . . . . . . . . . . . 26 
Linear Model . . . . . . . . . . . . . . . . . . . . . . 27

Exponential Model . . . . . . . . . . . . . . . . . 28

3.2.2 Patch Size and Search Window Size _. . . . . . . . . . . . 28

3.2.3 The two-step approach . . . . . . . . . . . . . . . 37

3.3 Summary of the selected parameters . . . . . . . . . . . . . . . . 39

4 Experimental Results and Analysis $\quad 40$

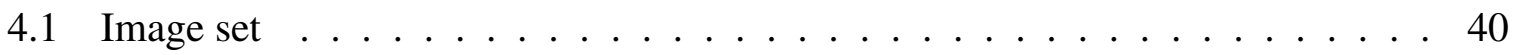

4.2 Noise Generation . . . . . . . . . . . . . . . . . . . . 40

4.3 Performance Measure . . . . . . . . . . . . . . . . . . . . . . . . 42

4.3.1 Peak Signal to Noise Ratio (PSNR) _ . . . . . . . . . . . . . . 43

4.3.2 Mean Structural Similarity (MSSIM) . . . . . . . . . . . . . . 43

4.4 Results and Analysis _ . . . . . . . . . . . . . . . . . 45

4.4.1 Performance evaluation using PSNR . . . . . . . . . . . . 45

4.4.2 Performance evaluation using MSSIM . . . . . . . . . . . . 48

4.4 .3 Visual Comparison . . . . . . . . . . . . . . . 51

4.4 .4 Intensity Profile $\ldots \ldots \ldots \ldots$

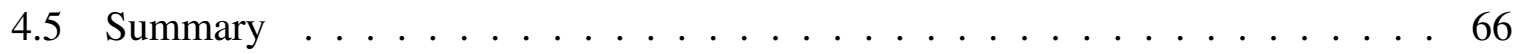

5 Conclusion and Future Work $\quad 67$

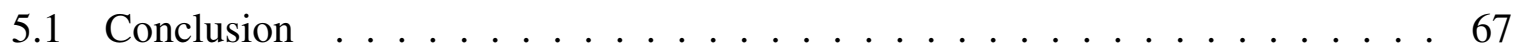

5.2 Future Work . . . . . . . . . . . . . . . . . . . 68

$\begin{array}{lr}\text { Bibliography } & 69\end{array}$

$\begin{array}{ll}\text { Curriculum Vitae } & 74\end{array}$ 


\section{List of Figures}

2.1 Probability density function of Gaussian random variable . . . . . . . . . 5

2.2 Image affected with additive white Gaussian noise (a) Noise-free image. (b) Image affected with Gaussian noise $(\sigma=40) \ldots \ldots . \ldots 6$

2.3 BM3D Block Diagram . . . . . . . . . . . . . . . . . . . . . 14

3.1 Training image set (a) Lena. (b) Barbara (c) Peppers (d) Baboon (e) Boats . . . 26

3.2 Bar chart of PSNR for different linear models (changing the coefficient $a$ ) . . 27

3.3 Bar chart of PSNR comparison between different exponential models (changing coefficient $a) \ldots \ldots \ldots \ldots \ldots$

3.4 Bar chart of PSNR comparison between different search window size . . . . . . 30

3.5 PSNR comparison between different patch sizes (a) Noise, $\sigma 5$ - 50 (b) Noise,

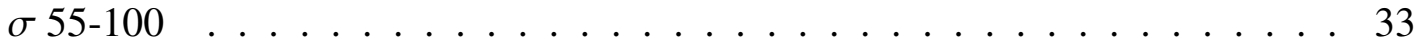

3.6 Plot of optimal search window for various noise levels . . . . . . . . . . . 35

3.7 Curve fitting using a linear model . . . . . . . . . . . . . . 35

3.8 Curve fitting using a quadratic model . . . . . . . . . . . . . . 36

3.9 Curve fitting using a exponential model . . . . . . . . . . . . . . 36

3.10 Bar chart of PSNR comparison of proposed method for multiple steps . . . . 38

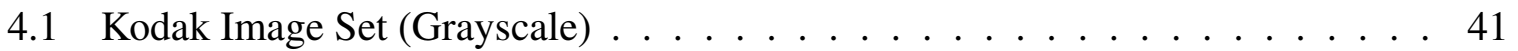

4.2 Example of Gaussian noise $(\sigma=40)$ added to a noise-free image . . . . . . . . 42

4.3 Bar chart of PSNR comparison of proposed and existing methods . . . . . . . . 47

4.4 Bar chart of MSSIM comparison of proposed and existing methods . . . . . . 50 
4.5 Visual Comparison for $\sigma=20 \ldots \ldots \ldots \ldots \ldots \ldots$

4.6 Visual Comparison for $\sigma=40 \ldots \ldots \ldots \ldots \ldots$

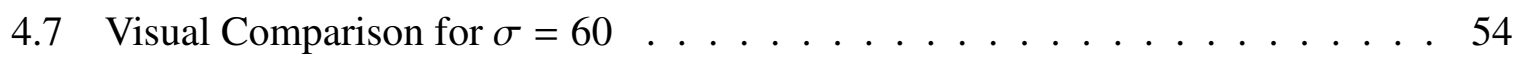

4.8 Visual Comparison for $\sigma=80 \ldots \ldots \ldots \ldots \ldots \ldots$

4.9 Visual Comparison for $\sigma=100 \ldots \ldots \ldots \ldots \ldots$

4.10 Visual Comparison (Zoom) for $\sigma=20 \ldots \ldots \ldots \ldots \ldots$. . . . . 57

4.11 Visual Comparison (Zoom) for $\sigma=40 \ldots \ldots \ldots \ldots$

4.12 Visual Comparison (Zoom) for $\sigma=60 \ldots \ldots \ldots \ldots$

4.13 Visual Comparison (Zoom) for $\sigma=80 \ldots \ldots \ldots \ldots$. . . . . . 60

4.14 Visual Comparison (Zoom) for $\sigma=100 \ldots \ldots \ldots$. . . . . . . . 61

4.15 Row number 100 of Girl image used for generating intensity profile. Scan line shown as a red line. . . . . . . . . . . . . . 63

4.16 Intensity Profile Comparison of the Girl image at scan line $100(\sigma=40) \ldots 64$

4.17 Intensity Profile Comparison of the Girl image at scan line $100(\sigma=80) \ldots 65$ 


\section{List of Tables}

3.1 PSNR Comparison of linear models for different coefficient $a$. . . . . . . . . . 27

3.2 PSNR Comparison between exponential models for different coefficient $a$. . . 29

3.3 PSNR Comparison by changing the search window size . . . . . . . . . . 30

3.4 PSNR Comparison for fine tuning the search window size . . . . . . . . . . 31

3.5 PSNR Comparison by changing the patch size . . . . . . . . . . . . . 32

3.6 PSNR Comparison by fixing patch size $=9 \times 9$ and changing search window size 34

3.7 PSNR comparison of proposed method for multiple steps for various noise levels 38

4.1 PSNR comparison of the Girl image among the proposed method, the NLM method, variants of NLM and BM3D denoising scheme for various noise levels 46

4.2 PSNR comparison of the proposed method, the NLM method, variants of NLM and BM3D denoising scheme for various noise levels . . . . . . . . . . . 46

4.3 Standard deviation of PSNR values of proposed method and existing methods for different noise levels . . . . . . . . . . . . . . . . . . . . . 47

4.4 MSSIM comparison of the Girl image among the proposed method, the NLM method, variants of NLM and BM3D denoising scheme for various noise levels

4.5 MSSIM comparison of the proposed method, the NLM method, variants of NLM and BM3D denoising scheme for various noise levels . . . . . . . . . . . 49

4.6 Standard deviation of MSSIM values of proposed method and existing methods for different noise levels . . . . . . . . . . . . . . . . . 50 
4.7 Pearson correlation coefficient comparison of the proposed method, the noisy image, the NLM method, variants of NLM and BM3D denoising scheme for noise $\sigma=40 \ldots \ldots \ldots \ldots \ldots$

4.8 Pearson correlation coefficient comparison of the proposed method, the noisy image, the NLM method, variants of NLM and BM3D denoising scheme for

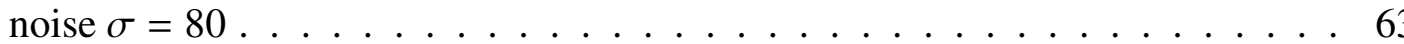




\section{Chapter 1}

\section{Introduction}

A digital image is made of finite number of elements, called pixels and can be defined as a two dimensional function. For grayscale images, the pixel intensity, also referred to as grayscale value, takes on values between 0 and 255, where 0 represents the darkest pixel and 255 represents the brightest pixel.

The pixels of a digital image can be contaminated with noise which results in random variation of brightness in the image. The noise is introduced during the image acquisition phase due to image sensors, in low light environment, or during transmission. Image denoising is the process of estimating the original image from a given noisy image and is one of the most fundamental problems in image processing. For an imaging application, working with noisy images can significantly affect the performance and reliability of the overall system. Image denoising is used as a pre-processing step in imaging applications to reduce the amount of noise and a major challenge is to reduce as much noise as possible while still keeping most of the visual information, such as edges and textures. 


\subsection{Motivation}

Earlier approaches to image denoising consisted of methods which attempt to estimate the denoised value of a pixel based on the information in a local area around each pixel. While such methods have been successful, to some extent, the resulting denoised images are often blurry. Buades et al. proposed the Non-Local Means (NLM) denoising scheme [3][4], using a nonlocal patch based concept. It attempts to identify self-similarities in a noisy image and calculate a weighted average of similar pixels in order to estimate a denoised image. Although many variants has been proposed after the original non-local means scheme, there are still room for improvements. The underlying nature of non-local means using all pixels in a defined search area tends to deviate the estimated denoised pixel value from the true noise-free intensity. In addition, the key parameters of the original NLM are kept fixed, irrespective of the amount of noise in the noisy image. One of our focus is to propose a variant of the non-local means scheme for better denoising performance. Another focus is to empirically define a set of models for determining the value of the key parameters, based on the noise level of a given image.

\subsection{Thesis Contribution}

The original non-local means denoising scheme considers all the pixels, defined within a search area, for calculating the weighted average of the denoised image. This includes all the pixels which are not similar to the reference pixel being denoised. Even with low weights, the pixels which are not similar to the reference pixel being denoised will move the estimated denoised intensity value from the true intensity. In our proposed method, we have thresholded the pixel weights against a cut-off weight to use only a subset of the available pixels for calculating the weighted average. In the original non-local means and its variants, the search window size and patch size are fixed, irrespective of the level of noise. In our proposed method, we have defined models for determining the optimal search window and patch size for a given noise 
level. The models are determined using a learning based approach. We have also applied our proposed method as a two-step process, where the basic denoised output of the first step is further processed to remove remaining noise artifacts, especially at high noise levels.

\subsection{Thesis Outline}

The thesis is divided into five chapters. The current introductory chapter is Chapter 1, where we have outlined the objectives and thesis contributions. In Chapter 2, we discussed the background of image denoising in details. In Chapter 3, we introduced our proposed method in details and explained the parameters selection procedure that we adopted in our proposed method. In Chapter 4, we presented the experimental results of our proposed method and compared them to that of the existing image denoising schemes. Finally, in Chapter $\mathbf{5}$ we listed our concluding remarks and future works. 


\section{Chapter 2}

\section{Background}

\subsection{Image Noise}

Noise is a random signal which corrupts the original signal by adding unwanted information to the signal. In a digital image, noise is a random variation of brightness. It is usually produced during the image acquisition phase, caused by the sensors of digital cameras or scanners. Modern digital cameras have come a long way in using high quality sensors which have significantly reduced the presence of noise during image acquisition yet, noise still can affect the quality of an image, especially in low light conditions.

Digital noise types include: Additive noise, Salt and Pepper Noise and Multiplicative noise.

\subsubsection{Additive Noise}

Additive noise gets added with the image signal. An image affected by additive noise can be modeled using:

$$
v(i)=u(i)+n(i)
$$

where $v(i)$ is the observed gray value at pixel $i, u(i)$ is the noise-free gray value and $n(i)$ is the amount of noise at pixel $i$. 


\section{White Gaussian Noise}

White noise is a random signal with a constant power spectral density [9]. Gaussian noise is a statistical noise which follows the Gaussian distribution. The probability density function of a Gaussian random variable, $z$, is modeled as shown:

$$
p(z)=\frac{1}{\sqrt{2 \pi} \sigma} e^{-\frac{(z-\mu)^{2}}{2 \sigma^{2}}}
$$

where $z$ represents gray level, $\mu$ is the mean, $\sigma$ is the standard deviation of the distribution, also referred to as the noise density of the Gaussian noise signal, and $\sigma^{2}$ is the variance. Figure 2.1 shows the probability density distribution of a Gaussian model. Almost all the area under the curve lies between $\mu \pm 3 \sigma$. Figure 2.2 shows an example of an image which is affected by additive white Gaussian noise with $\sigma=40$.

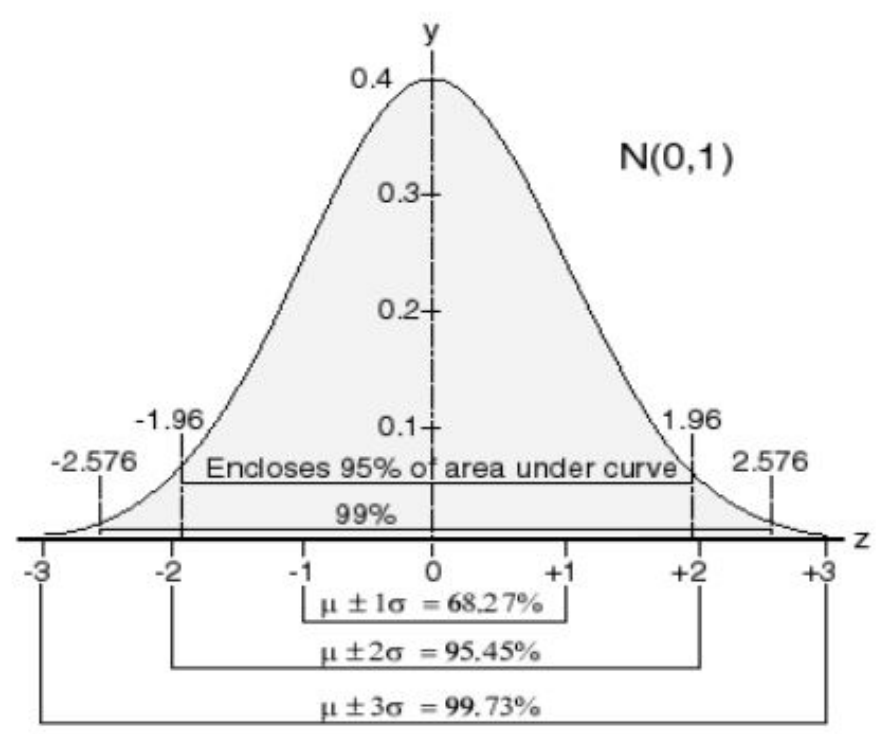

Figure 2.1: Probability density function of Gaussian random variable 


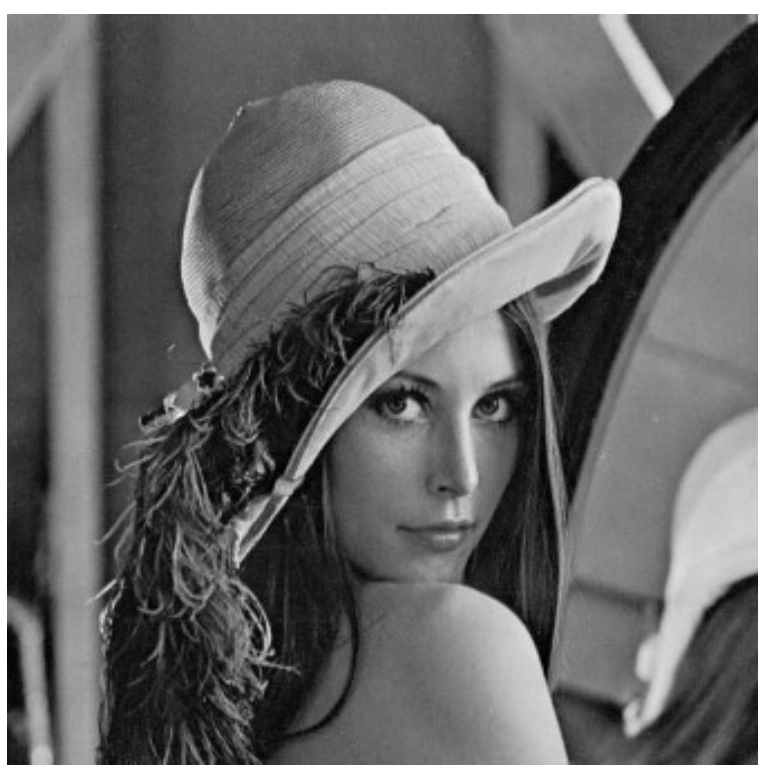

(a)

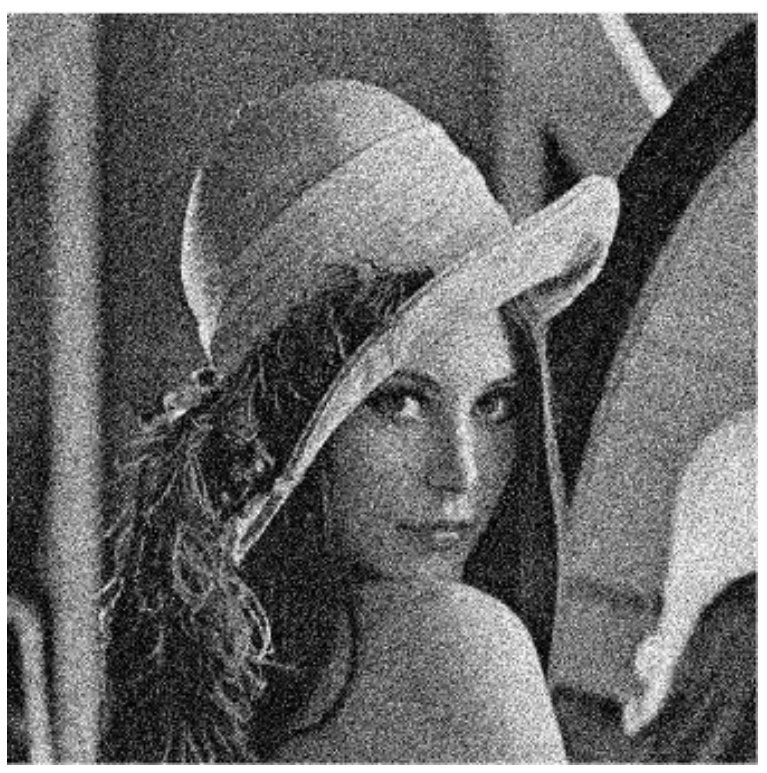

(b)

Figure 2.2: Image affected with additive white Gaussian noise (a) Noise-free image. (b) Image affected with Gaussian noise $(\sigma=40)$

\section{Estimating additive white Gaussian noise}

For optimal denoising of digital images affected with additive Gaussian noise, most methods require some knowledge of the noise density, $\sigma$. Although the exact noise density may not be possible to be known beforehand, a good estimation can be calculated. One of the methods for estimating noise is the local variance method [1]. For estimating the noise density, a given noisy image is divided in small sub-blocks. The gray value variance is calculated for each sub-block and the variance of all the sub-blocks are averaged to estimate the noise variance of the Gaussian noise. The noise density is the square root of the average variance. The size of the sub-images used for calculating the value of $\sigma$ should ideally be the smallest possible block size (e.g., $2 \times 2$ ). Increasing the block size starts to overshoot the estimation of the noise density, compared to the actual noise density. 


\subsubsection{Multiplicative Noise}

Multiplicative noise is a random signal which gets multiplied to the original signal. Multiplicative noise can be modeled as shown:

$$
v(i)=u(i) \times n(i),
$$

where $v(i)$ is the observed gray value for pixel $i, u(i)$ is the noise-free value and $n(i)$ is the noise at pixel $i$. Most common form of multiplicative noise is speckle noise. Common methods for removing speckle noise include Lee filter [18] and Frost filter [8].

\subsubsection{Salt \& Pepper Noise}

Salt and Pepper noise is a form of impulse noise where the gray level of the affected pixels are either changed to the maximum possible intensity level (255 in case of 8-bit grayscale images) or to the minimum possible intensity value (0). The noise appears as white and black pixels (or dots) in the image and the noise density, $d$, of the salt-and-pepper noise is determined by the number of pixels affected by the noise. It affects approximately $d \times N \times M$ pixels in an image, where $d$ satisfies the condition $0 \leq d \leq 1$ and $N \times M$ is the number of pixels in the image.

\subsection{Image Denoising}

Most imaging system have an image acquisition phase and the performance of the overall system depends on the quality of the image. The level of noise in the image plays a critical role in the outcome of the system and it is desirable to have as much reduced noise as possible during the image acquisition phase. Any noise added during this phase is first denoised before using the image in the system. The main challenge of a denoising scheme is to reduce noise 
while preserving the texture and edges of the image. A scheme which is able to reduce most of the noise but completely blurs the entire image to a point where minimal visual information can be extracted is not ideal. Similarly a denoising method which preserves a lot of the texture in the image but fails to remove the noise to a satisfactory level is not an effective method as well.

The image denoising methods can be classified primarily into two groups, based on the approach they use for reducing noise effect: spatial domain approaches and frequency domain approaches

\subsubsection{Spatial Domain Approach}

The term spatial domain refers to the image plane itself [9]. In this domain, the raw observed gray values of a noisy image are manipulated for estimating the denoised image. It takes advantage of the notion that pixels in a small local neighborhood are correlated. Spatial domain approaches can be denoted by the expression $g(x, y)=T[f(x, y)]$, where $f(x, y)$ is an input image, $g(x, y)$ is the output, or, processed image and $T$ is some operator defined over a neighborhood of $(x, y)$.

The neighborhood around an image pixel at position $(x, y)$ is defined as a rectangular or square sub-image centered at position $(x, y)$. The size of the window is selected based on the operation performed over the selected sub-image. The operator $T$ is usually applied over sub-

images centered on each pixels in the given image. The resulting processed image is considered as the denoised image.

\subsubsection{Frequency Domain Approach}

In frequency domain approaches, the image is transformed to the frequency domain using, for example, Fourier transform or wavelet transform. The frequency domain separates smooth regions in an image into low frequencies, while edges and subtle information into high fre- 
quencies, thus making it easier to target and enhance certain regions in an image.

\subsection{Spatial Domain Methods}

This section covers some of the common spatial domain denoising methods. The spatial domain methods tries to estimate the denoised images based on the raw intensity value of image pixels.

\subsubsection{Mean Filter}

The mean filter is a method of smoothing the image to reduce the noise in the image. The mean filter operates by replacing the pixels in the image with the mean/average of the pixels in a local neighborhood, including itself [9]. This helps reduce the pixel intensities which are unrepresentative of its neighborhood. Mean filter is a convolution method and is based on a kernel. The size of the kernel determines the degree of smoothing around each pixel. The weights assigned to each position in the kernel can either be equal or can be weighted based on the proximity to the center of the kernel. For example, if weights are assigned following a Gaussian model then the pixels closer to the center will have more weights than the pixels further from the center and the sum of the weights equals 1.

\subsubsection{Median Filter}

The median filter looks to remove noise by estimating the noise-free gray value based on the median of the intensities of the pixels around the local neighborhood of a reference pixel [9]. A $N \times N$ window is centered around each pixels in the image and the pixel intensity of the center is replaced by the median gray value of the pixels within the window. Compared to the mean filter, median filter is more robust to the presence of outliers within the local window. Unlike the mean filter, where the outliers affect the average intensity value of the patch, the median 
ignores outliers. The median filter is also used for denoising salt-and-pepper noise.

\subsubsection{Gaussian smoothing}

Gaussian smoothing is a 2-D spatial operator used to blur an image to remove noise. It is based on a convolution kernel where the coefficients of the kernel followed a 2-D Gaussian distribution. The kernel is used for averaging the gray value of a local neighborhood centered at position $(x, y)$ and the coefficients represents the weight assigned to each pixel position in the window. Center pixel has the most importance and the weights assigned to the pixels in the neighborhood reduces as the distance between the corresponding pixel and the center increases. The isotropic Gaussian distribution, with variance $\sigma^{2}$, is shown:

$$
G(x, y)=\frac{1}{2 \pi \sigma^{2}} e^{-\frac{x^{2}+y^{2}}{2 \sigma \sigma^{2}}}
$$

\subsubsection{Anisotropic Diffusion}

The filtering approaches mentioned earlier use only the raw pixel values in a small local neighborhood around each pixels to determine the denoised image. These methods do not take into account the extent to which the neighborhood overlaps with smooth or textured regions. Thus the use of such linear filters are detrimental for edge and texture preservation, resulting in blurry denoised images. To mitigate the detail-loss issue of the isotropic averaging, Perona and Malik proposed the iterative Anisotropic Diffusion approach [23]. It aims to reduce noise without removing the fine details and edges in the images which are important for visual interpretation by leveraging multiscale description of given noisy image. It involves embedding the original image into a set of derived images $I(x, y, t)$ by convolving the original image $I_{0}(x, y, t)$ with a Gaussian kernel $G(x, y ; t)$ with variance $t$

$$
I(x, y, t)=I_{0}(x, y, t) * G(x, y ; t)
$$


The authors identified some criteria which any method for generating multiscale descriptions of images must satisfy:

1) Casuality: It was first pointed out by Witkin and Koenderink in [29][16] and it states that a scale-space representation should not generate any spurious details when moving from fine to coarser scales.

2) Immediate Localization: At each resolutions the region boundaries should be sharp and should coincide with the semantically meaningful boundaries at that resolution

3) Piecewise Smoothing: At all scales the intra-region smoothing should occur preferentially over inter-region smoothing.

The authors proposed to use a non-linear smoothing model instead of a linear model, such as Gaussian smoothing. In this model, the level of smoothing applied on each pixel is adjusted based on the pixel position in a region. If a pixel is part of a smooth intra-region area more blurring is applied to remove noise, but if the pixel is part of an edge pixel the smoothing is not applied. This ensures that the smoothing occurs only in intra-region areas and ignore the intraregion areas in the image. To reduce the noise around edges, anisotropic diffusion is applied. Instead of taking the Laplacian of an image to calculate the divergence of gradient in the image, a new function $g()$ is multiplied by the gradient of the image and the divergence of the result is taken. The function $g()$ calculates the degree of diffusion to be applied on the edges. If the edge is strong the diffusion level is weak and if the edge is weak then it is diffused more.

\subsubsection{2-D Adaptive Wiener Filter}

The conventional Wiener filter operates in the frequency domain (covered later in Section 2.4.2). The Adaptive Wiener Filter proposed by Jin et al. [14] uses adaptive weighted averaging for calculating the second order statistics required by the Wiener filter. The objective of Wiener filter is to minimize the mean squared error between the restored and the actual undegraded image. 
In the spatial domain, the objective function of the Wiener filter can be expressed as:

$$
\operatorname{MSE}(\hat{x})=\frac{1}{N} \sum_{i, j=1}^{N}(\hat{x}(i, j)-x(i, j))^{2}
$$

When $x(i, j)$ and the noise are stationary Gaussian processes, the Wiener filter is the optimal filter [17] and the Wiener filter has a simple scalar form:

$$
\hat{x}(i, j)=\frac{\sigma_{x}^{2}(i, j)}{\sigma_{x}^{2}(i, j)+\sigma_{n}^{2}(i, j)}\left[y(i, j)-\mu_{x}(i, j)\right]+\mu_{x}(i, j),
$$

where, $\sigma^{2}$ and $\mu$ are the signal variance and means respectively. The mean of the noise is zero.

\subsection{Frequency Domain Methods}

This section covers some frequency domain methods, namely the Low-Pass Filter, Wiener Filter and Block Matching and 3D Filtering methods.

\subsubsection{Low-Pass Filter}

A low-pass filter is a filter that allows signals to pass unattenuated with frequency lower than a certain threshold/cut-off frequency [9]. Frequencies higher than the threshold are attenuated. In digital images contaminated with additive noise, the random added signal is represented by high frequencies. A low-pass filter reduces the effect of the additive noise by removing high frequencies from the image. A simple low-pass filter is represented by the given expression:

$$
H(k, l)=\left\{\begin{array}{l}
1 \quad \text { if } \sqrt{k^{2}+l^{2}}<f_{\text {threshold }} \\
0 \quad \text { if } \sqrt{k^{2}+l^{2}}>f_{\text {threshold }}
\end{array}\right.
$$

In an image, the edges and fine details of an image are also translated to high frequencies due to high gradient changes in pixel intensities around edges and lines, and using the low-pass 
filter can soften the edges and textures and result in a blurred image. The amount of blurring depends on the threshold frequency used by the low-pass filter.

\subsubsection{Wiener Filter}

Wiener filter is a transform domain approach used for recovering images affected with image noise and image degradation, such as blur. The Wiener filter model attempts to restore images by minimizing the Mean Squared Error (MSE) between the restored image and the original image [9]. The objective function is shown below:

$$
e^{2}=E(f-\hat{f})^{2}
$$

where $f$ is the original image, $\hat{f}$ is the restored image and the operator $E$ is the expected mean squared error. The Wiener operates in the frequency domain of an image and the minimum objective function is given as:

$$
\hat{F}(u, v)=\frac{H^{*}(u, v)}{|H(u, v)|^{2}+\frac{S_{n}(u, v)}{S_{f}(u, v)}} G(u, v),
$$

The terms in the equation are:

$H(u, v)=$ degradation function

$H^{*}(u, v)=$ complex conjugate of $\mathrm{H}(\mathrm{u}, \mathrm{v})$

$S_{n}(u, v)=$ power spectrum of the noise

$S_{f}(u, v)=$ power spectrum of the undegraded image

If the power spectrum of the noise is zero, i.e., the image is noise-free, the Wiener filter reduces to an inverse filter. For a given noisy image, the power spectrum of the undegraded image is difficult, if not impossible, to estimate. In case of such uncertainty the expression is 
approximated, as shown below:

$$
\hat{F}(u, v)=\frac{H^{*}(u, v)}{|H(u, v)|^{2}+K} G(u, v),
$$

where $K$ is a constant.

\subsubsection{BM3D}

In the field of image denoising, specifically for white Gaussian noise, a popular method has emerged for tackling the problem of image noise called Block Matching and 3D Filter (BM3D). It was proposed by K.Dabov et al. in 2006 [6]. BM3D demonstrated superior denoising performance compared to the existing methods and have attracted the attention of researchers working in the image of image enhancement and restoration.

BM3D is a 2-step process and has been inspired by the non-local concept first introduced in Non-Local Means (NLM). A block diagram illustrating the steps of BM3D is shown in Figure 2.3 .

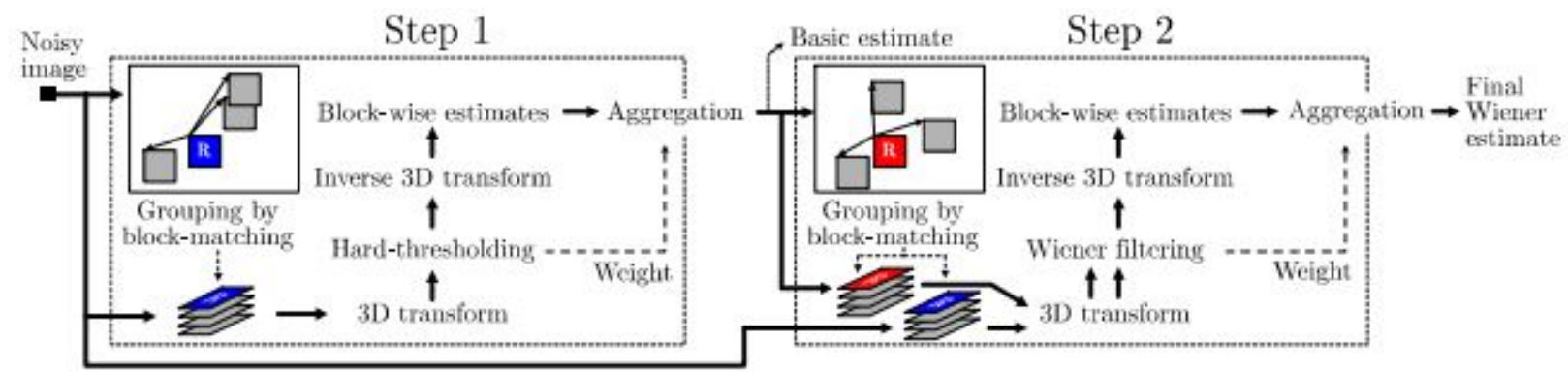

Figure 2.3: BM3D Block Diagram

In the first step, the process starts by defining a local neighborhood, also referred to as the reference patch and searches for similar patches inside a search window, usually defined as a bounded area around the reference patch. The similarity between the search patch and the reference patch is decided based on a certain threshold. If the similarity is above the threshold, the search patch is marked as one of the similar patches. After calculating the similarity be- 
tween the patches inside the bounded search area, all the similar patches are stacked together, building a 3D block. BM3D applies some 3D transformations on the block to transform from spatial domain to frequency domain. After the 3D transformation, the resulting coefficients are thresholded, called hard thresholding, where coefficients below a certain threshold are reduced to zero. The block coefficients are transformed back to the spatial domain, using inverse 3D transform. Next, BM3D generates a basic estimate of the denoised image from the block which has been inverse transformed. For estimating the values of the reference patch, all the patches in the 3D block are aggregated and it works by assigning different weights while estimating the pixel values in the reference patch.

In the second step of $\mathrm{BM} 3 \mathrm{D}$ another grouping of patches are carried out, similar to that of the first step. This time however the grouping of patches into 3D blocks are done based on the basic estimate obtained from the first step. After 3D blocks are generated by selecting similar patches around the reference patch, the block is transformed using a 3D transformation. Based on the transformed coefficients the restored image coefficients are estimated using the restoration concept used in Wiener filter. The Wiener shrinkage coefficients are calculated for the $3 \mathrm{D}$ blocks, followed by inverse transform to revert back to the spatial representation of the 3D block. Finally the Wiener shrinkage coefficients are multiplied by the 3D block to get the denoised representation of the block. 


\subsubsection{BM3D Extensions}

BM3D managed to demonstrate state-of-the-art performance compared to the previous denoising models and after the method was initially proposed, researchers have continued to work with the BM3D model to improve it's denoising performance.

\section{BM3D Denoising using Shape-Adaptive Principal Component Analysis}

Dabov et al. proposed BM3D-SAPCA [7], an improvement over the regular BM3D using shape adaptive image patches and applied Principal Component Analysis (PCA) on the adaptive shape neighborhood as part of the 3D transform on blocks. In the first step, it performs shape adaptive grouping to find similar blocks and calculates the PCA basis of the 3D blocks. The remaining steps, including 3D transform and hard thresholding, are carried out similar to the one conventional BM3D.

\section{BM3D Denoising using SSIM Optimized Wiener Filter}

Hasan and El-Sakka [11] proposed an improvement in the Wiener filter optimized for maximizing the expected SSIM between the restored image and the original image. The improvement in the underlying Wiener filter also helps to improve the performance of BM3D as it uses the Wiener filter in the second phase of the denoising process. The main motivation was the observation that the mean squared error used in Wiener filter measures the absolute difference in intensity between two images and it may occur than the mean squared error can have similar values even when the two images are visually very different. To include some visual information when denoising an image, the objective function of the Wiener filter is changed from minimizing the expected MSE to maximizing the structural similarity between the restored and the original noise-free image. 


\subsection{Non-Local Means}

Buades et al. proposed a non-local based approach for image denoising [3][4]. Images have redundant or similar patterns in them and the Non-Local Means (NLM) approach attempts to take advantage of such self-similarities to estimate the denoised gray value of each pixel. Instead of using only a local region around each pixel for estimating the actual intensity of the pixel, NLM uses a non-local approach by searching for similar patches, within a certain search-bound, in the image. The center pixel of each patch contributes to a weighted averaging based on the similarity between the reference and search patches.

When comparing the reference patch to a search patch, a variation of the Euclidean distance is measured. The Euclidean distance measures the sum of squared difference between each pixel in a patch. To give more importance to pixels near the center of the patch, a Gaussian weight distribution is used, thus resulting in the final measurement being the weighted Euclidean distance, $\|N(i)-N(j)\|_{2, a}^{2}$, where $a$ is the standard deviation of the Gaussian kernel and $N(i)$ and $N(j)$ are the patches around pixel $i$ and $j$, respectively. The weight associated with each of the search patches is based on the similarity with the reference patch. After calculating the Euclidean distance between the patches, the weight is assigned using:

$$
w(i, j)=\frac{1}{Z(i)} e^{-\frac{\left\|v\left(N_{i}\right)-v\left(N_{j}\right)\right\|_{2, a}^{2}}{h^{2}}},
$$

where $Z(i)$ is the normalizing constant as defined by:

$$
Z(i)=\sum_{j} e^{-\frac{\left\|v\left(N_{i}\right)-v\left(N_{j}\right)\right\|_{2, a}^{2}}{h^{2}}}
$$

The constant, $h$, controls the decay rate of the exponential weight function. Given a noisy image, the estimated value $N L[v](i)$, for pixel $i$, is computed as a weighted average of the center pixels of the patches in a certain search area, see Equation (2.13). 


$$
N L[v](i)=\sum_{j \in I} w(i, j) v(j)
$$

where $w(i, j)$ is the weight calculated based on the similarity of neighborhood around pixel $i$ and $j$.

\subsection{Non-Local Means Variants}

The Non-Local Means(NLM) approach gained popularity due to its superior denoising results compared to other existing spatial domain denoising models and researchers have continued to work on the NLM approach to tackle some of it's limitations. Some extensions have been proposed to improve the computational cost.

\subsubsection{Principal Components of Non-Local Means}

Tasdizen used PCA to improve the computational time of non-local means [26]. The image patches which are used in a given search space around a reference pixel are first projected to a lower-dimensional subspace using PCA. The neighborhood similarity weights are then calculated from the reduced subspace instead of the original full space. Let $M$ be the number of pixels in a neighborhood $N$ and let $\left\{b_{p}\right\}_{p=1}^{M}$ be the eigenvector of the $M \times M$ empirical covariance matrix for the set of all neighborhood vectors $\left.\left\{v(N)_{j}\right)\right\}_{j=1}^{Q}$ where $Q$ denotes the total number of pixels in the image. The projections of the image neighborhood vectors onto the d-dimensional PCA subspace is shown below:

$$
v[d]\left(N_{i}\right)=\sum_{p=1}^{d} \underbrace{\left\langle v\left(N_{i}\right), b_{p}\right\rangle}_{f_{p}\left(N_{i}\right)} b_{p},
$$


where $f_{p}\left(N_{i}\right)$ is the length of the $i^{\text {th }}$ vector's projection onto the $p^{\text {th }}$ basic vector. Due to orthonormality of the basis

$$
\left\|v[d]\left(N_{i}\right)-v[d]\left(N_{j}\right)\right\|^{2}=\sum_{p=1}^{d}\left(f_{p}\left(N_{i}\right)-f_{p}\left(N_{j}\right)\right)^{2}
$$

The estimator for $d$ is defined as:

$$
\hat{u}[d](i)=\sum_{j \in S_{i}} \frac{1}{Z_{d}(i)} e^{-\frac{\sum_{p=1}^{d}\left(f_{p}\left(N_{i}\right)-f_{p}\left(N_{j}\right)\right)^{2}}{h^{2}}} v(j)
$$

The proposed approach with $d=M$ is equivalent of the standard non-local means.

\subsubsection{Improved Non-Local Means based on dimensionality reduction}

Maruf and El-Sakka [20] proposed to improve the computational time of the original nonlocal means algorithm by projecting image patches into a global feature space followed by a dimensionality reduction using statistical $t$-test. In a preprocessing step, the global feature space is generated by linearizing the patches to form a row vector of size $j$ where $j$ is the number of pixels in the patch. The total size of the matrix $C$ is $N \times j$ where $N$ is the total number of pixels in the image. After generating this matrix in the preprocessing step, a paired t-test of the null hypothesis is computed on the matrix, where the hypothesis tries to accept or reject a feature (i.e., an entire column in matrix $C$ ). After reducing the dimension of the initial feature vector, the reduced vector is used for calculating the similarity between two patches. The weighted averaging is based on the reduced feature space and thus reduces the exhaustive search over all patches in a search region, as done in the standard non-local means method. This achieves a reduction in the computation time of the algorithm.

Along with improvements in terms of the running time of the algorithm, the proposed methods have also tried to improve the denoising performance of non-local means. 


\subsubsection{SSIM-based Non-Local Means}

Rehman and Wang have proposed a SSIM based approach to non-local means [24]. For calculating the similarity between patches, the mean-squared error (MSE) may not always reflect the true similarity between patches. As MSE measures the squared difference between the pixel values, it may occur that two very different patches can have the same MSE values when compared to the reference patch. The resulting denoised image may not have the best perceptual quality. The SSIM-based approach uses the structural similarity to measure the likelihood of similarity between the reference and the search patch. The weighted average is calculated as shown:

$$
\hat{X}(i)=\frac{\sum_{j \in N_{i}} w_{S S I M}(i, j) Y^{\prime}(j)}{\sum_{j \in N_{i}} w_{S S I M}(i, j)},
$$

where $N_{i}$ denotes the set of patches in the search area defined around pixel $i$ and $W_{S S I M}(i, j)$ is the SSIM weight between patch $i$ and $j$.

The proposed method needed to address two major concerns. First, when calculating the structural similarity between patches, especially in high noise levels, the SSIM value may end up comparing the noise between the patches instead of the underlying structure. To overcome this concern, the authors proposed a way to estimate the noise in the patches and deduct the estimated noise from the pixel intensity values before calculating the structural similarity.

The second issue to address is that two structurally similar patches may have different contrast and mean values. To avoid the bias in mean and contrast, the patches are normalized before the weighted averaging step.

\subsubsection{Iterative Non-Local Means}

Bronx and Cremer [2] proposed an iterative non-local means approach. The non-local means method is applied on an image in iterative mode. In each iteration, the similarity between patches are calculated based on the result of the previous iteration. After calculating the 
weights, the weighted averaging is done by multiplying the weights of a patch with it's center gray value in the noisy version of the image. The proposed method did well on regular textured image but in non-regular textured image the resulting denoising image lost texture and significant blurring is observed.

\subsubsection{Non-Local Euclidean Median}

Chaudhury and Singer [5] proposed the Non-Local Euclidean Median, extending the concept of the original Non-Local Means scheme. The method is derived from the observation that the median is more robust to outliers than the mean. In the presence of noise in the image, the weights averaged over all possible patches, especially in a search-bound defined around image edges and lines, will move the resulting mean towards the outliers. The mean is the minimizer of $\sum_{j} w_{j}\left\|P-P_{j}\right\|^{2}$ over all patches $P$. Non-Local Euclidean Median proposed the select the patch, $P$, which minimizes $\sum_{j} w_{j}\left\|P-P_{j}\right\|$ and replace the noisy pixel value at position $(x, y)$ in the image with the pixel value of the center of patch $P$.

\subsubsection{Two-stage Non-Local Means with adaptive smoothing parameters}

Zhu et al. [33] proposed a two-stage non-local means method with adaptive smoothing parameters. Based on the noise estimation of a given noisy image, smoothing parameter $h_{\text {basic }}$ for the first stage is selected automatically and the basic denoised image is computed, as shown:

$$
\hat{y}_{i, b a s i c}=\sum_{j} w_{i j, b a s i c} y_{j}
$$

where $w_{i j, b a s i c}$ is the weight depending on the similarity between patches $i$ and $j$ and satisfies the usual conditions $0 \leq w_{i j, \text { basic }} \leq 1$ and $\sum_{j} w_{i j, \text { basic }}=1$. The weight is calculated as show in Equation (2.19)

$$
w_{i j, b a s i c}=\exp \left(-\frac{1}{h_{\text {basic }}^{2}}\left\|P_{i}-P_{j}\right\|^{2}\right)
$$


where $P_{i}$ and $P_{j}$ are the patches centered on pixel $i$ and $j$ and $h_{b a s i c}$ is the smoothing parameter which controls the decay rate of the exponential function. For the first stage the smoothing parameter is set as $h_{i j, b a s i c}=0.75 \times \sigma$.

Most of the image noise is removed after the first stage but for high noise levels some noise artifacts still remain in the image, thus the basic image is refined one more time. The resulting $\hat{y}_{\text {basic }}$ image of the first stage is again denoised using the non-local means method but using different smoothing parameters $h_{\text {final }}$. The final image is computed as shown in Equation (2.20)

$$
\hat{y}_{i, f i n a l}=\sum_{j} w_{i j, f i n a l} \hat{y}_{i, b a s i c}
$$

Similar to the first stage, the weights between patch $i$ and $j$ are calculated as:

$$
w_{i j, \text { final }}=\exp \left(-\frac{1}{h_{\text {final }}^{2}}\left\|P_{i}-P_{j}\right\|^{2}\right)
$$

In the second step of the process, the smoothing parameter is defined as:

$$
h_{\text {final }}=\left\{\begin{array}{cc}
\frac{\sigma^{2}}{100}, & \sigma<30 \\
0.5 \sigma, & \sigma \geq 30
\end{array}\right.
$$

\subsection{Non-Local Means Applications}

Non-local means and its variants have been used in various imaging applications such as medical imaging, including MRI brain images [13], CT scan imaging [15] and 3D ultrasound imaging [12]. It is also used in video denoising [10] [30], surface salinity detection [32] and metal artifact detection [22]. 


\section{Chapter 3}

\section{Methodology}

The non-local means method defines a search area of size $S \times S$ centered on the pixel, $i$, being denoised. The similarity of all the patches, defined around each of the pixels, within the search area is considered during the weighted averaging process, where higher weights are assigned to patches which are more similar, as determined by lower euclidean distance to the reference patch. The goal of the weighted averaging process is to estimate the true noise-free intensity value of pixel $i$, based on the similarity of the patches within the defined search area of a given noisy image. The inclusion of center pixels of the patches which have low weights is likely to deviate the resulting estimate from the true pixel intensity value of the denoised image.

\subsection{Adaptive Non-Local Means using Weight Thresholding}

In our proposed method, only a subset of the available patch centers are considered for the estimation of the denoised pixel. The patches are selected based on the similarity measure compared to the reference patch. Effectively, a cut-off weight, $w_{\text {thresh }}$ is selected using a defined percentile value, $w_{\text {percentile }}$ among the available patch weights within the bounded search area and the weights of the patches are thresholded against $w_{\text {thresh }}$. All weights above $w_{\text {thresh }}$ are unchanged and weights below $w_{\text {thresh }}$ are reduced to zero, thus removing their pixel centers 
from the weighted averaging process. The selected percentile is adjusted based on the noise level of the given image. In real systems, the actual amount of noise in a noisy image cannot be known beforehand. The noise can be estimated in digital image using fuzzy processing techniques [25], image filters [21] and local variance estimate method [19]. In this research, we used the local variance estimation method (see Section 2.1.1).

For low noise levels, a higher cut-off weight, $w_{\text {thresh }}$, is selected for thresholding the patch weights and as the noise level of a given image increases, $w_{\text {thresh }}$ is lowered to include more patch centers for averaging. For lower noise levels, only the patches with high similarity measure to a reference patch can be used to estimate a denoised image. The remaining patches can be considered outliers. So, a higher cut-off threshold is selected for low noise levels. In high noise, the Euclidean distance measurement may not give a true measure of patch similarity as it will end up comparing, to some extent, the noise between patches along with the structures of the patches. So, considering only the higher weighted patch centers, by keeping the threshold value high, can in fact deviate the denoised estimation from the true value. To mitigate this effect, the threshold value is lowered so that more pixels are averaged for attenuating the noise. The denoised image is calculated as shown below:

$$
N L[v](i)=\sum_{j \in I} \hat{w}(i, j) v(j),
$$

where $\hat{w}(i, j)$ is the thresholded weight between patch at pixel $i$ and patch at pixel $j$ as shown:

$$
\hat{w}(i, j)= \begin{cases}w(i, j), & \text { if } w(i, j)>w_{\text {thresh }} \\ 0, & \text { otherwise }\end{cases}
$$

Non-local means has two key parameters, namely the patch size and the search size. These parameters are kept constant irrespective of the amount of noise in the image. In our proposed method we have attempted to select the optimal patch and search window size based on the 
noise level in the image. Through a iterative learning approach, we have empirically defined models for selecting the patch size and the corresponding search window size for any noise level, $\sigma$. The models were determined empirically using an iterative learning approach on a training image set.

The proposed method is applied in a two-step approach. In the first step, the proposed method is used to generate a basic estimate of the denoised image. In the basic estimate, most of the noise is reduced but still some visible noise artifacts remain, especially for stronger noise levels and it is necessary to further denoise the basic image for better denoising [31]. As most of the noise is reduced in the basic image, similar regions can be identified more easily which helps to generate better denoised images in the second step. In the second step, the basic image is denoised using a similar method utilized in the first step, but with smaller smoothing parameters. To verify that the two-step approach is sufficient, we conducted experiments to measure the improvement in the denoising performance with further steps and found the amount of improvements to be negligible. The results of this experiment is shown in Section 3.2.3

\subsection{Parameter Selection}

The primary parameters in the proposed method are:

1. Cut-off weight for thresholding

2. Patch size

\section{Search window size}

The parameters are determined empirically, based on experiments conducted on a set of test images. The performance measure of tuning each of the parameters are used to define the models for the parameters. The set of test of images is shown in Figure 3.1. The training images are selected to address common image characteristics, including smooth regions, textured regions 
and fine details. The Lena and Peppers images have smooth regions, while Barbara, Boats and Baboon images has lot more texture and fine details. All the PSNR values reported are averaged after repeating each of the experiments 10 times.

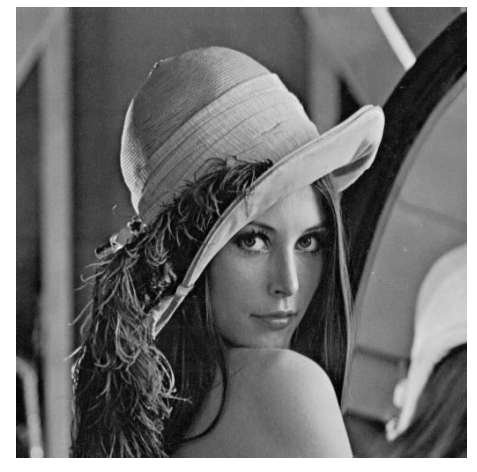

(a)

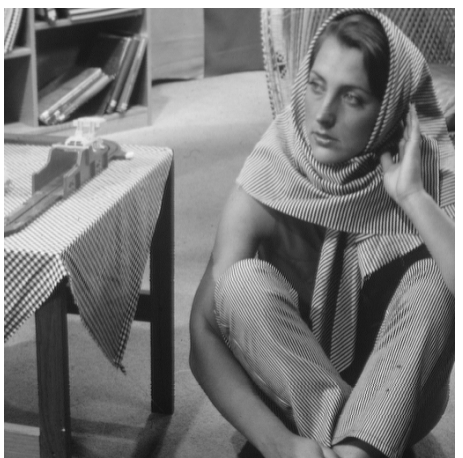

(b)

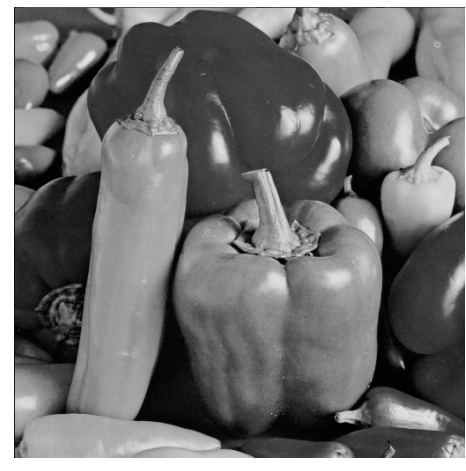

(c)

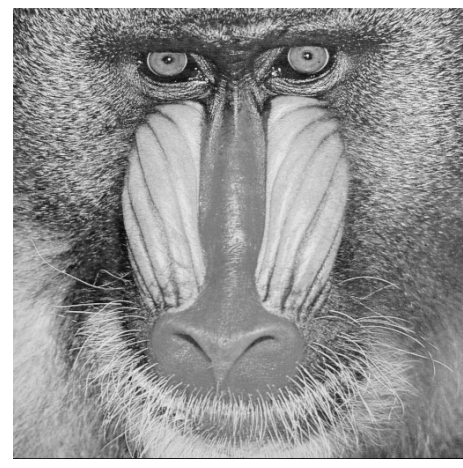

(d)

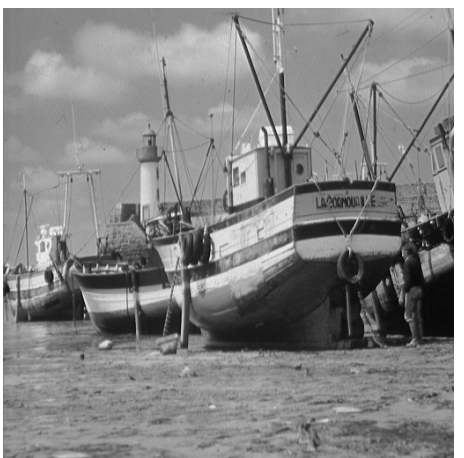

(e)

Figure 3.1: Training image set (a) Lena. (b) Barbara (c) Peppers (d) Baboon (e) Boats

\subsubsection{Cut-off Weight for Thresholding}

The patch size and search window size are fixed and the weights are thresholded based on the cut-off weight percentile, $w_{\text {percentile }}$, determined by the model. For the purpose of determining the suitable thresholding model, the patch size is fixed at $7 \times 7$ and the search window size fixed at $11 \times 11$. Two different models are analyzed, the linear model and the exponential model. 
Table 3.1: PSNR Comparison of linear models for different coefficient $a$

\begin{tabular}{|c|c|c|c|}
\hline Noise $(\sigma)$ & $a=0.25$ & $a=0.5$ & $a=1$ \\
\hline 10 & 33.43 & 33.75 & $\mathbf{3 3 . 8 7}$ \\
\hline 20 & 31.85 & 32.12 & $\mathbf{3 2 . 2 5}$ \\
\hline 30 & 28.74 & 29.06 & $\mathbf{2 9 . 1 5}$ \\
\hline 40 & 26.63 & 26.91 & $\mathbf{2 6 . 9 8}$ \\
\hline 50 & 24.71 & 24.96 & $\mathbf{2 5 . 0 7}$ \\
\hline 60 & 24.00 & $\mathbf{2 4 . 2 8}$ & 23.95 \\
\hline 70 & 23.08 & $\mathbf{2 3 . 3 3}$ & 22.75 \\
\hline 80 & 22.75 & $\mathbf{2 3 . 1 4}$ & 22.57 \\
\hline 90 & 21.87 & $\mathbf{2 2 . 2 8}$ & 21.64 \\
\hline 100 & 21.48 & $\mathbf{2 1 . 7 1}$ & 21.22 \\
\hline Average & 25.85 & $\mathbf{2 6 . 1 5}$ & 25.94 \\
\hline
\end{tabular}

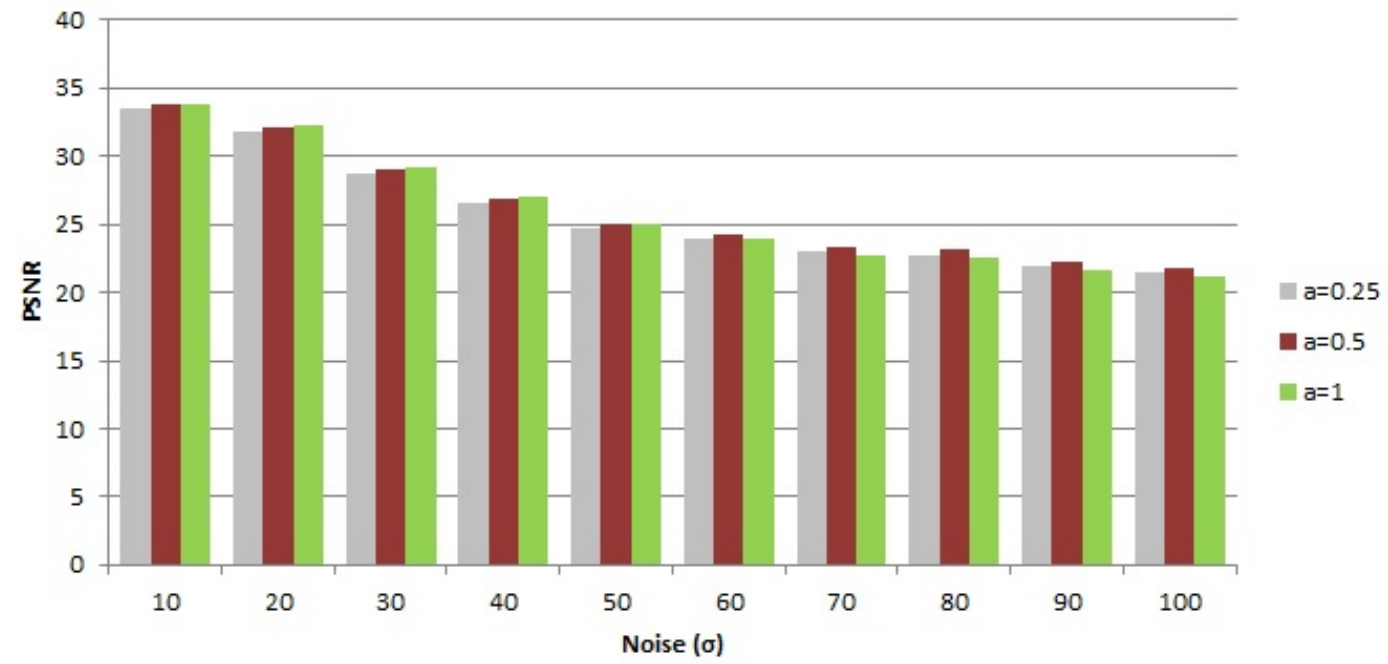

Figure 3.2: Bar chart of PSNR for different linear models (changing the coefficient $a$ )

\section{Linear Model}

The linear model for determining the cut-off weight percentile is defined as:

$$
w_{\text {percentile }}=\operatorname{ceil}(100-a \sigma),
$$

where $\operatorname{ceil}()$ rounds a decimal value to the smallest following integer, coefficient $a$ is a constant and $\sigma$ is the standard deviation of Gaussian noise.

Different linear models are defined by changing the value of coefficient $a$. The PSNR 
comparison of using different linear models for weight thresholding is tabulated in Table 3.1. Figure 3.2 shows the bar chart of PSNR comparison between various linear models. For noise levels $\sigma \leq 50$, a coefficient value of $a=1$ generate better results and for $\sigma>50$, the coefficient value $a=0.5$ demonstrate better performance.

\section{Exponential Model}

The exponential model for determining the cut-off weight percentile is defined as:

$$
w_{\text {percentile }}=\operatorname{ceil}\left(100 \times e^{-0.01 a \sigma}\right),
$$

where $\operatorname{ceil}($ ) rounds a decimal value to the smallest following integer, coefficient $a$ is a constant and $\sigma$ is the standard deviation of Gaussian noise.

The result of using the exponential model for determining the cut-off weight is shown is Table 3.2. Figure 3.3 shows the bar chart of PSNR comparison between different exponential models. For the exponential model, the coefficient value of $a=1$ demonstrate better results for all noise levels. The average performance, as well as the noise-wise performance, of the exponential model, with coefficient $a=1$, has better denoising performance compared to the linear models. So, the exponential model, with coefficient $a=1$ is selected for determining the cut-off weight percentile for any given noise level, $\sigma$.

\subsubsection{Patch Size and Search Window Size}

For varying the search window size, the patch size is fixed at $7 \times 7$. The weights are thresholded using the exponential model, selected in previous section. The result of varying the search window is shown in Table 3.3. The table shows that for $\sigma<50$ the search window size $11 \times 11$ perform optimally and for $\sigma \geq 50$ the search size $21 \times 21$ performs best. To help determine a model for selecting the search window size, the denoising performance on the test images 
Table 3.2: PSNR Comparison between exponential models for different coefficient $a$

\begin{tabular}{|c|c|c|c|c|}
\hline Noise $(\sigma)$ & $a=0.5$ & $a=1$ & $a=2$ & $a=4$ \\
\hline 10 & 33.77 & $\mathbf{3 3 . 8 9}$ & 33.68 & 33.34 \\
\hline 20 & 32.09 & $\mathbf{3 2 . 2 2}$ & 31.96 & 31.62 \\
\hline 30 & 29.13 & $\mathbf{2 9 . 3 6}$ & 29.09 & 28.8 \\
\hline 40 & 26.94 & $\mathbf{2 7 . 1 1}$ & 26.86 & 26.57 \\
\hline 50 & 24.90 & $\mathbf{2 5 . 1 3}$ & 24.79 & 24.68 \\
\hline 60 & 24.16 & $\mathbf{2 4 . 3 5}$ & 24.06 & 23.85 \\
\hline 70 & 23.28 & $\mathbf{2 3 . 4 8}$ & 23.15 & 23.01 \\
\hline 80 & 23.06 & $\mathbf{2 3 . 2 4}$ & 22.87 & 22.66 \\
\hline 90 & 22.21 & $\mathbf{2 2 . 3 4}$ & 21.92 & 21.74 \\
\hline 100 & 21.89 & $\mathbf{2 2 . 0 5}$ & 21.68 & 21.42 \\
\hline Average & 26.14 & $\mathbf{2 6 . 3 2}$ & 26.01 & 25.77 \\
\hline
\end{tabular}

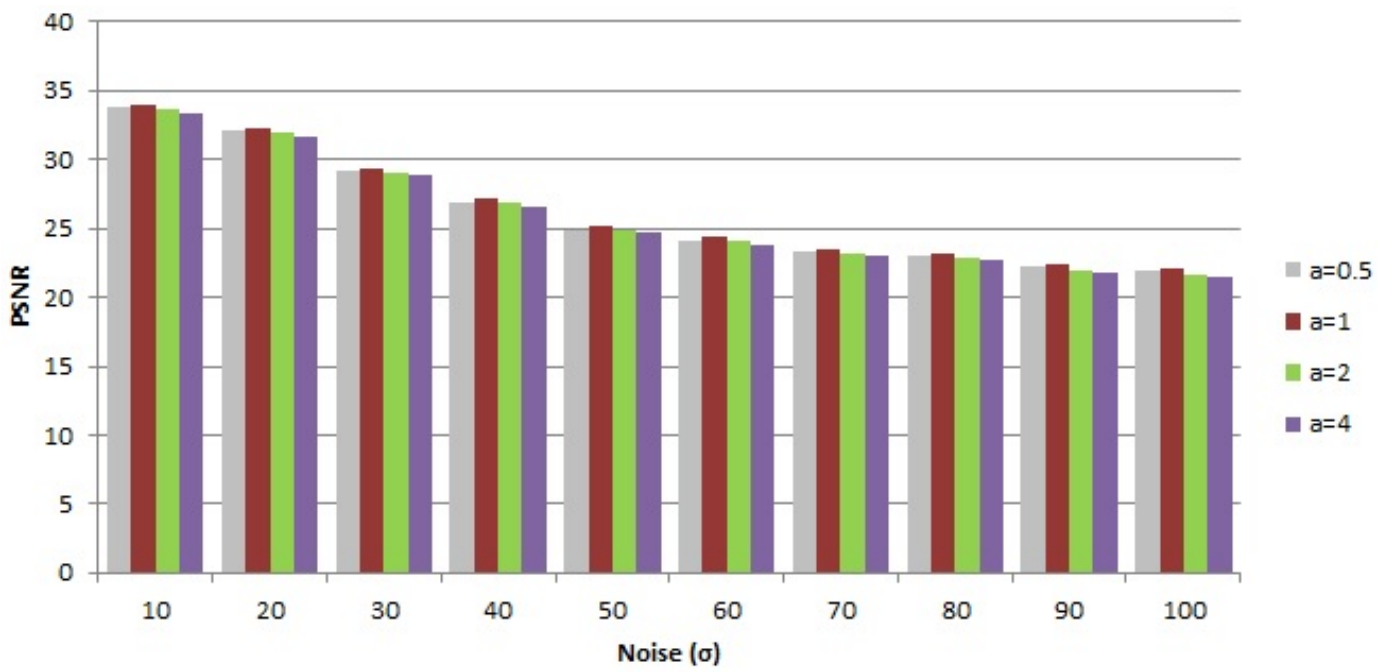

Figure 3.3: Bar chart of PSNR comparison between different exponential models (changing coefficient $a$ )

are again measured by varying the search window size, but this time using odd-integer window sizes only between $11 \times 11$ and $21 \times 21$, while using more noise levels.

The patch size and search window size for a given noise level are determined empirically, using an iterative learning approach on a training image set. At first, the patch size is fixed and the search window size is varied, for each noise levels, to select the best search window size. The noise levels, $\sigma$, ranges from 10 to 100 , with a step size equals 5 . Next, the patch size is varied for each noise levels, while using the best search window size for each noise as 
Table 3.3: PSNR Comparison by changing the search window size

\begin{tabular}{|c|c|c|c|c|}
\hline Noise $(\sigma)$ & $5 \times 5$ & $11 \times 11$ & $21 \times 21$ & $35 \times 35$ \\
\hline 10 & 33.26 & $\mathbf{3 4 . 1 9}$ & 33.94 & 33.40 \\
\hline 20 & 31.44 & $\mathbf{3 2 . 4 8}$ & 31.66 & 31.28 \\
\hline 30 & 28.83 & $\mathbf{2 9 . 3 5}$ & 28.68 & 28.27 \\
\hline 40 & 27.44 & $\mathbf{2 8 . 1 2}$ & 28.06 & 27.44 \\
\hline 50 & 23.79 & 24.56 & $\mathbf{2 5 . 0 5}$ & 24.40 \\
\hline 60 & 22.84 & 23.70 & $\mathbf{2 4 . 2 7}$ & 23.91 \\
\hline 70 & 22.15 & 23.09 & $\mathbf{2 3 . 7 3}$ & 23.48 \\
\hline 80 & 21.66 & 22.56 & $\mathbf{2 3 . 4 1}$ & 23.19 \\
\hline 90 & 20.73 & 21.57 & $\mathbf{2 2 . 8 8}$ & 22.42 \\
\hline 100 & 20.35 & 21.06 & $\mathbf{2 2 . 5 2}$ & 22.15 \\
\hline Average & 25.25 & 26.07 & $\mathbf{2 6 . 4 2}$ & 25.99 \\
\hline
\end{tabular}

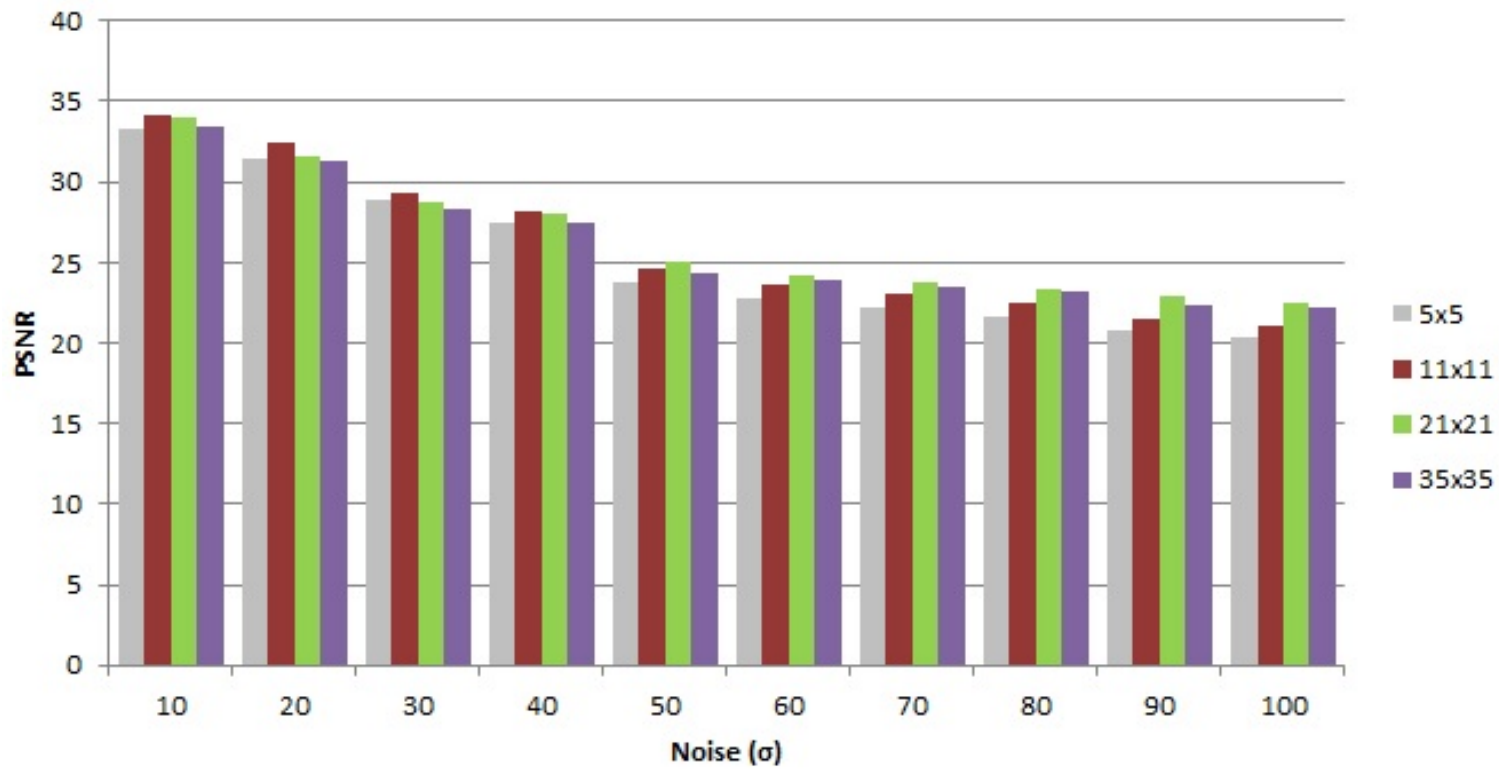

Figure 3.4: Bar chart of PSNR comparison between different search window size

determined in the previous step. The best patch size for each noise level is used to find the corresponding optimal search window sizes one more time. This process is repeated until an iteration is reached where updating the optimal search window size for a noise level did not change the corresponding best patch size and vice versa.

To determine the patch and search window size models, the patch size is initially fixed at $7 \times 7$ and the search window size is varied. The average PSNR comparison of the various search window size is shown in Table 3.4. 
Table 3.4: PSNR Comparison for fine tuning the search window size

\begin{tabular}{|c|c|c|c|c|c|c|}
\hline Noise $(\sigma)$ & $11 \times 11$ & $13 \times 13$ & $15 \times 15$ & $17 \times 17$ & $19 \times 19$ & $21 \times 21$ \\
\hline 5 & $\mathbf{3 7 . 0 9}$ & 36.76 & 36.54 & 36.30 & 36.14 & 36.08 \\
\hline 10 & $\mathbf{3 4 . 1 6}$ & 34.02 & 33.84 & 33.75 & 33.61 & 33.43 \\
\hline 15 & $\mathbf{3 2 . 9 6}$ & 32.88 & 32.73 & 32.64 & 32.48 & 32.23 \\
\hline 20 & $\mathbf{3 2 . 5 0}$ & 32.36 & 32.23 & 32.18 & 31.88 & 31.65 \\
\hline 25 & 30.32 & $\mathbf{3 0 . 4 3}$ & 30.28 & 30.13 & 30.05 & 29.98 \\
\hline 30 & 29.32 & $\mathbf{2 9 . 5 6}$ & 29.38 & 29.11 & 28.87 & 28.73 \\
\hline 35 & 28.54 & $\mathbf{2 8 . 6 7}$ & 28.46 & 28.29 & 27.98 & 27.86 \\
\hline 40 & 27.12 & 27.22 & $\mathbf{2 7 . 7 9}$ & 27.21 & 27.15 & 27.07 \\
\hline 45 & 26.04 & 26.32 & $\mathbf{2 6 . 6 8}$ & 26.48 & 26.31 & 26.14 \\
\hline 50 & 24.52 & 24.86 & 25.03 & $\mathbf{2 5 . 3 9}$ & 25.15 & 25.02 \\
\hline 55 & 24.13 & 24.43 & 24.75 & $\mathbf{2 5 . 0 7}$ & 25.81 & 25.70 \\
\hline 60 & 23.78 & 24.04 & 24.28 & $\mathbf{2 4 . 5 2}$ & 24.39 & 24.27 \\
\hline 65 & 23.11 & 23.54 & 23.98 & $\mathbf{2 4 . 2 9}$ & 24.01 & 23.76 \\
\hline 70 & 23.12 & 23.35 & 23.77 & $\mathbf{2 4 . 0 8}$ & 23.88 & 23.65 \\
\hline 75 & 22.84 & 23.07 & 23.29 & 23.54 & $\mathbf{2 3 . 8 3}$ & 23.66 \\
\hline 80 & 22.55 & 22.84 & 23.00 & 23.38 & $\mathbf{2 3 . 6 2}$ & 23.45 \\
\hline 85 & 22.07 & 22.22 & 22.45 & 22.88 & $\mathbf{2 3 . 1 0}$ & 22.96 \\
\hline 90 & 21.23 & 21.46 & 21.75 & 21.94 & 22.27 & $\mathbf{2 2 . 5 6}$ \\
\hline 95 & 20.94 & 21.10 & 21.39 & 21.74 & 22.05 & $\mathbf{2 2 . 2 8}$ \\
\hline 100 & 20.64 & 20.82 & 21.15 & 21.38 & 21.66 & $\mathbf{2 2 . 0 2}$ \\
\hline Average & 26.35 & 26.50 & 26.14 & $\mathbf{2 6 . 7 2}$ & 26.71 & 26.62 \\
\hline
\end{tabular}


Table 3.5: PSNR Comparison by changing the patch size

\begin{tabular}{|c|c|c|c|c|}
\hline Noise $(\sigma)$ & $3 \times 3$ & $5 \times 5$ & $7 \times 7$ & $9 \times 9$ \\
\hline 5 & 36.76 & 37.04 & $\mathbf{3 7 . 2 5}$ & 37.14 \\
\hline 10 & 33.64 & 33.86 & $\mathbf{3 4 . 1 2}$ & 33.96 \\
\hline 15 & 32.39 & 32.58 & $\mathbf{3 2 . 8 6}$ & 32.73 \\
\hline 20 & 31.92 & 32.22 & $\mathbf{3 2 . 4 5}$ & 32.36 \\
\hline 25 & 29.86 & 30.14 & $\mathbf{3 0 . 3 4}$ & 30.23 \\
\hline 30 & 29.07 & 29.43 & $\mathbf{2 9 . 6 1}$ & 29.47 \\
\hline 35 & 28.06 & 28.31 & $\mathbf{2 8 . 5 6}$ & 28.45 \\
\hline 40 & 27.21 & 27.50 & $\mathbf{2 7 . 7 4}$ & 27.59 \\
\hline 45 & 26.28 & 26.55 & $\mathbf{2 6 . 7 8}$ & 26.54 \\
\hline 50 & 24.99 & 25.29 & $\mathbf{2 5 . 4 6}$ & 25.35 \\
\hline 55 & 24.47 & 24.87 & $\mathbf{2 5 . 0 3}$ & 24.94 \\
\hline 60 & 24.13 & 24.42 & $\mathbf{2 4 . 6 0}$ & 24.46 \\
\hline 65 & 23.66 & 24.05 & $\mathbf{2 4 . 2 3}$ & 24.11 \\
\hline 70 & 23.48 & 23.81 & $\mathbf{2 4 . 0 2}$ & 23.95 \\
\hline 75 & 23.42 & 23.64 & $\mathbf{2 3 . 8 9}$ & 23.81 \\
\hline 80 & 23.09 & 23.37 & $\mathbf{2 3 . 5 5}$ & 23.52 \\
\hline 85 & 22.53 & 22.82 & 23.09 & $\mathbf{2 3 . 2 3}$ \\
\hline 90 & 22.08 & 22.45 & 22.63 & $\mathbf{2 2 . 8 2}$ \\
\hline 95 & 21.79 & 22.13 & 22.36 & $\mathbf{2 2 . 6 8}$ \\
\hline 100 & 21.35 & 21.78 & 21.97 & $\mathbf{2 2 . 4 1}$ \\
\hline Average & 26.51 & 26.81 & $\mathbf{2 7 . 0 3}$ & 26.99 \\
\hline & & & & \\
\hline
\end{tabular}

For the next step of determining the patch size to be used in our proposed method, the patch size is varied while using the optimal search window for a given noise level, as shown in Table 3.3. The result of changing the patch size is shown in Table 3.5. The results indicated that the patch size $7 \times 7$ works optimally for noise level $\sigma<85$ and for noise level $\sigma \geq 85$ the patch size $9 \times 9$ is optimal.

For noise level $\sigma \geq 85$, the optimal patch size is different than the patch size used to find the optimal search window size previously. The experiment for finding the optimal search window is again iterated, but this time only for noise levels $\sigma \geq 85$ and using a patch size $9 \times 9$. The PSNR comparison is shown in Table 3.6.

As the optimal search window size did not change, even with the updated patch size, the iteration is stopped. 


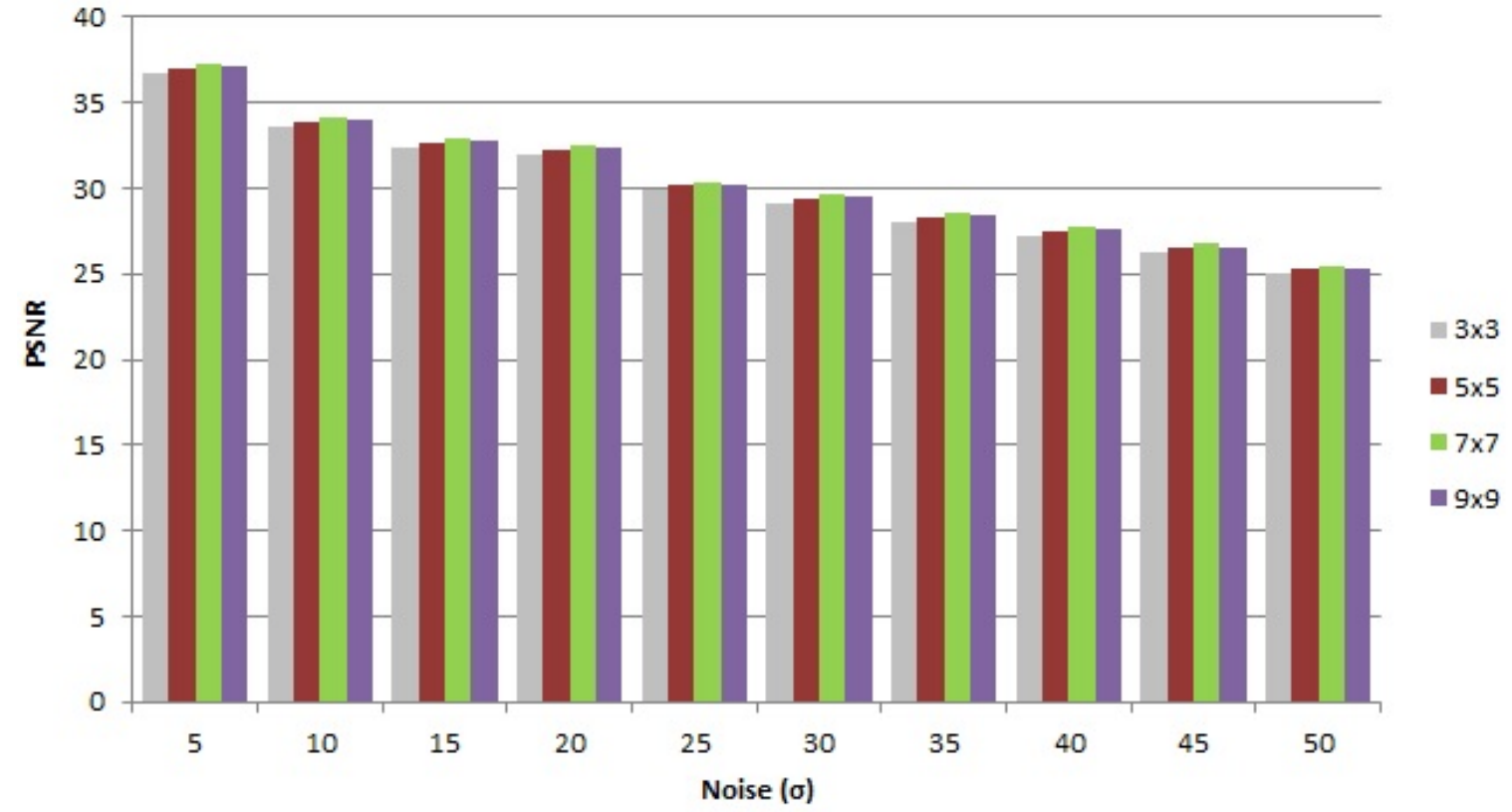

(a)

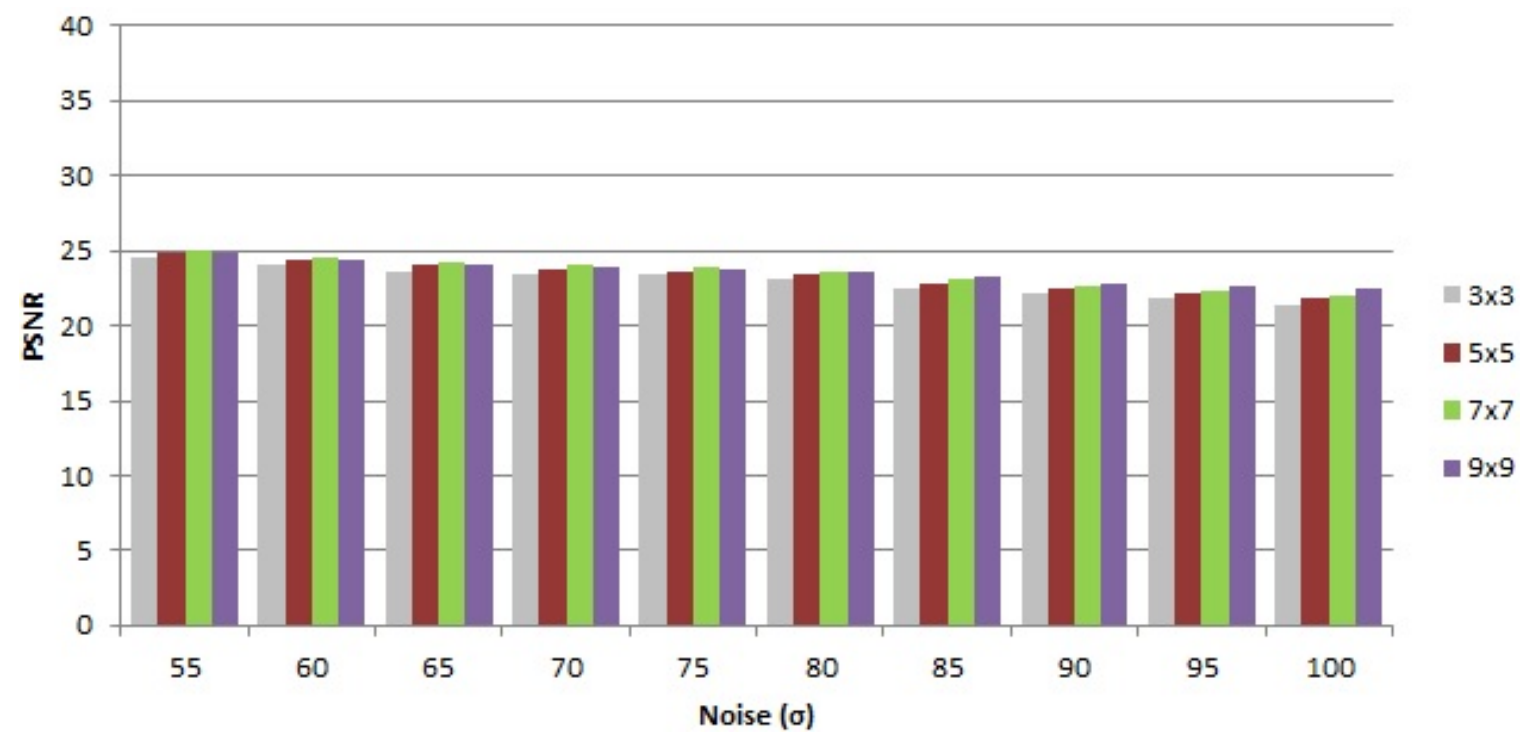

(b)

Figure 3.5: PSNR comparison between different patch sizes (a) Noise, $\sigma 5$ - 50 (b) Noise, $\sigma$ 55-100 
Table 3.6: PSNR Comparison by fixing patch size $=9 \times 9$ and changing search window size

\begin{tabular}{|c|c|c|c|c|c|c|}
\hline Noise $(\sigma)$ & $11 \times 11$ & $13 \times 13$ & $15 \times 15$ & $17 \times 17$ & $19 \times 19$ & $21 \times 21$ \\
\hline 85 & 22.28 & 22.51 & 22.74 & 23.02 & $\mathbf{2 3 . 2 7}$ & 23.14 \\
\hline 90 & 21.53 & 21.75 & 22.02 & 22.21 & 22.54 & $\mathbf{2 2 . 8 5}$ \\
\hline 95 & 21.24 & 21.47 & 21.82 & 22.19 & 22.48 & $\mathbf{2 2 . 6 4}$ \\
\hline 100 & 21.17 & 21.32 & 21.65 & 21.86 & 22.11 & $\mathbf{2 2 . 4 3}$ \\
\hline Average & 21.55 & 21.76 & 22.06 & 22.32 & 22.60 & $\mathbf{2 2 . 7 6}$ \\
\hline
\end{tabular}

The optimal search window sizes and the corresponding noise levels are plotted in a graph, as shown in Figure 3.6. The plotted points are used to determine a model, for the search window size, using a best-fit curve for the given points. Three different models are used to determine the best-fit curve:

\section{Linear Model}

\section{Quadratic Model}

\section{Exponential Model}

The best fit curve using a linear model is shown in Figure 3.7. The best fit curve for the quadratic model is shown in Figure 3.8 and the curve for the exponential model is shown in Figure 3.9. 


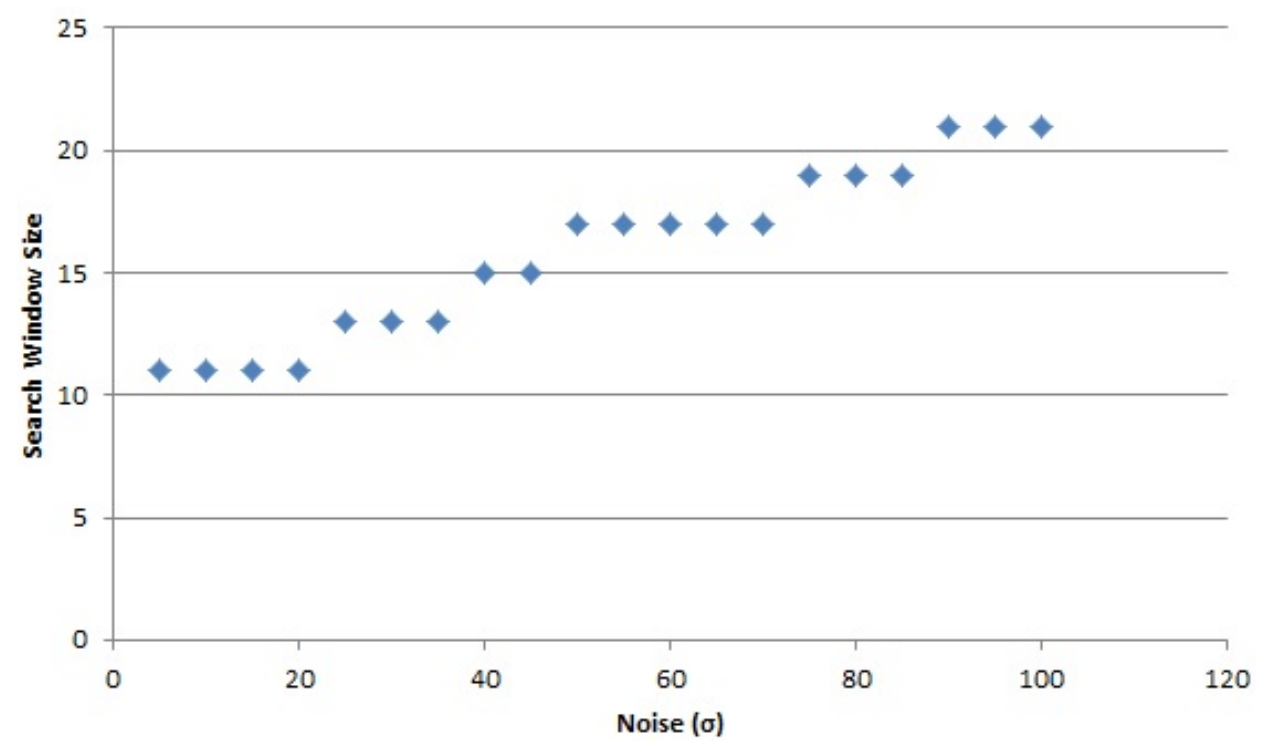

Figure 3.6: Plot of optimal search window for various noise levels

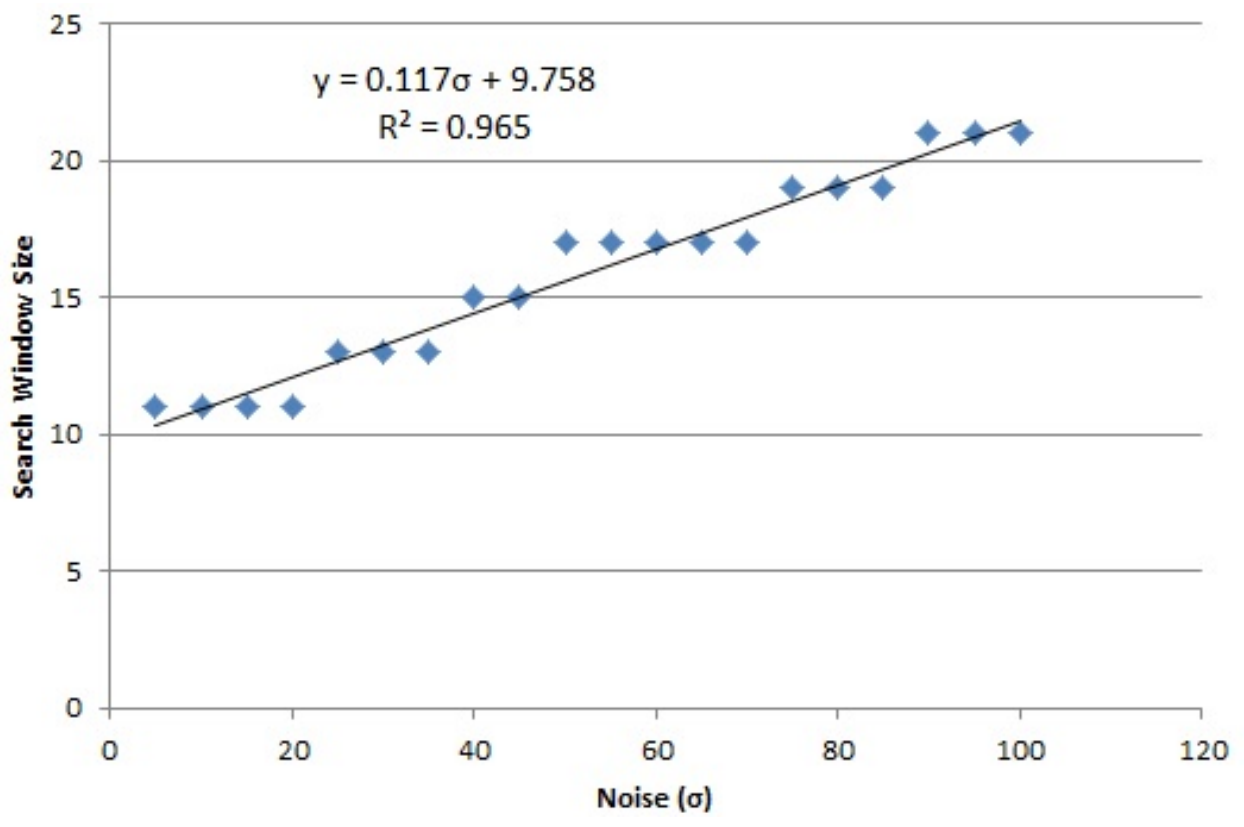

Figure 3.7: Curve fitting using a linear model 


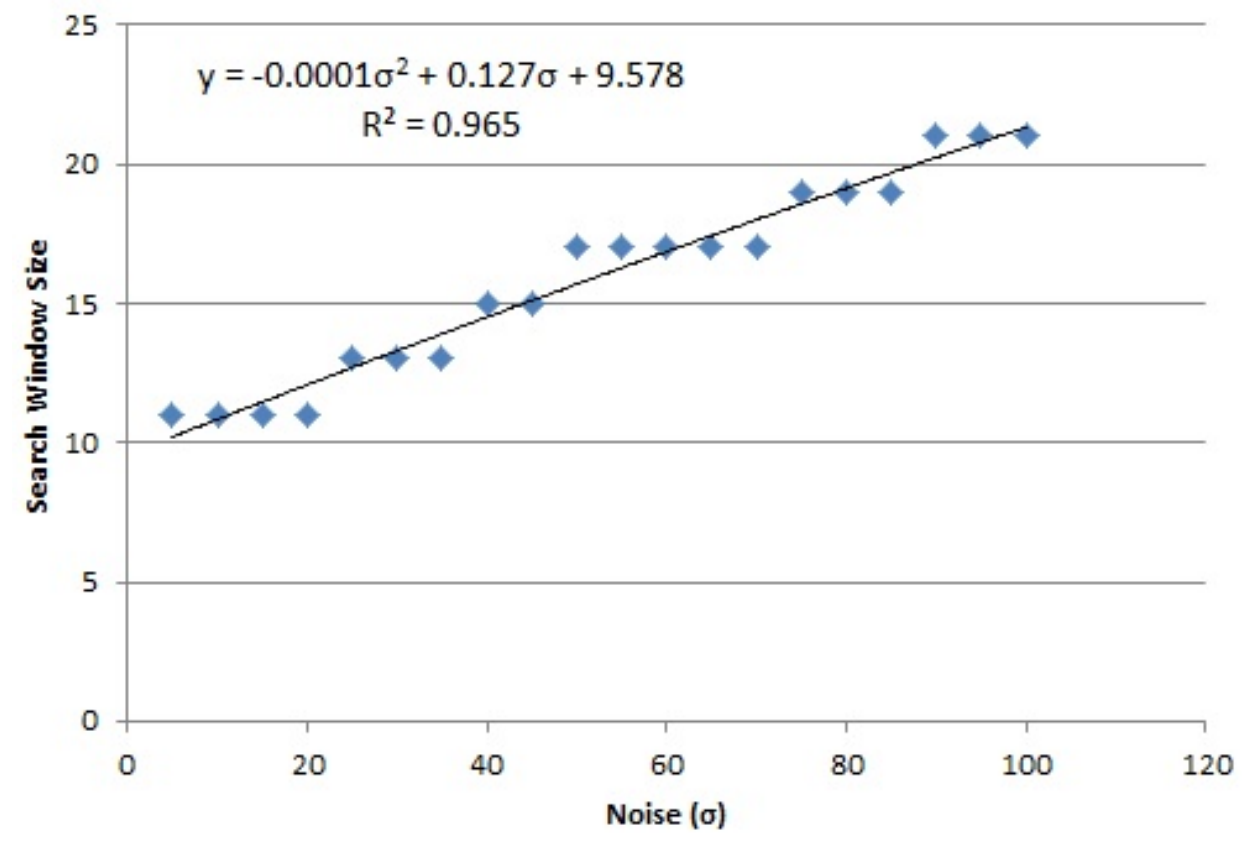

Figure 3.8: Curve fitting using a quadratic model

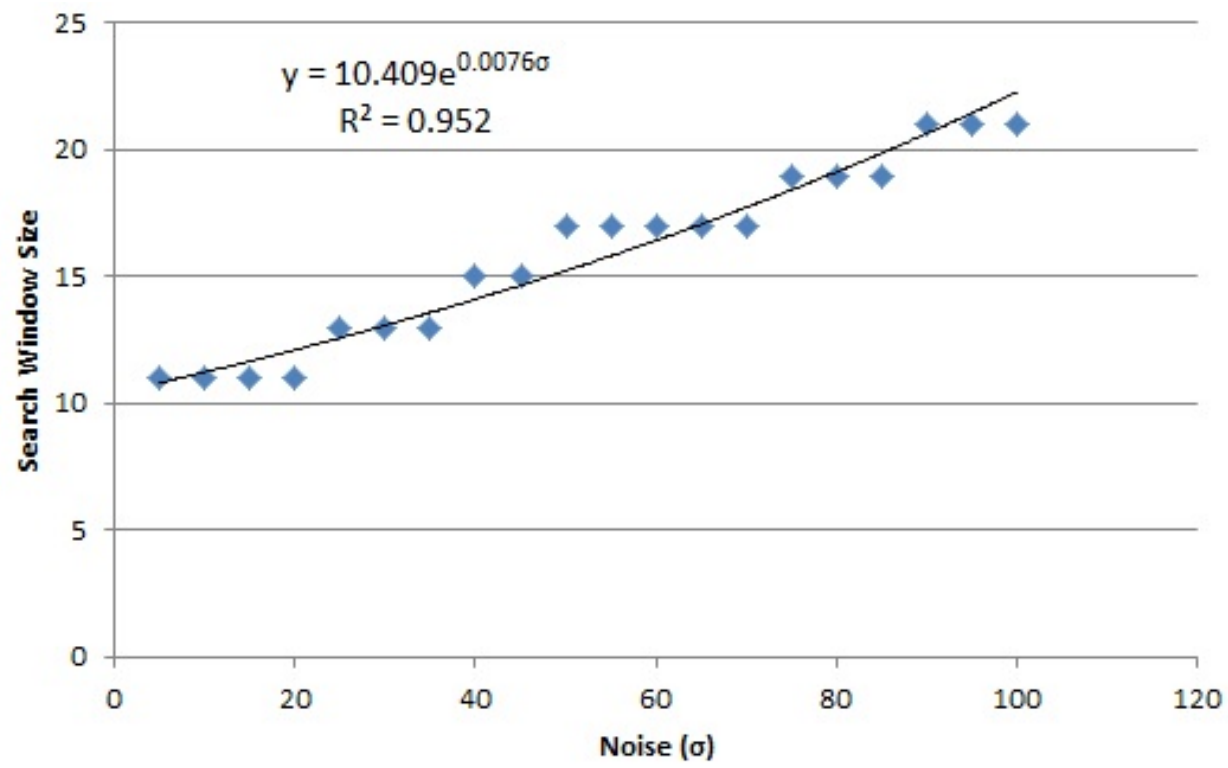

Figure 3.9: Curve fitting using a exponential model 
The goodness of each of the curve models is evaluated using the R-squared statistical measure. It measures how close the actual data points are to the fitted curve and the values range from 0.0 to 1.0 , where a higher value represents a better-fit curve. The equations and the R-squared values of the three models are:

1. Linear Model: $y=0.117 \sigma+9.758, \quad R^{2}=0.965$

2. Quadratic Model: $y=-0.0001 \sigma^{2}+0.127 \sigma+9.574, \quad R^{2}=0.965$

3. Exponential Model: $y=10.409 e^{0.008 \sigma}, \quad R^{2}=0.952$

Based on the R-squared values, the linear and quadratic models have very similar $R^{2}$ results. In the quadratic model, the coefficient of the $x^{2}$ term is close to 0 , making the model equation very similar to the linear model. The R-squared value of the exponential model is lower than that of the linear model and exponential functions are more computationally expensive than linear functions. Comparing the curve fittings of the three models, the linear model is selected for determining the search window size for any given noise level $\sigma$.

As the search window is centered around the pixel being denoised, it is required to use odd-numbered size for the search. For a given noise level, the result of the linear model is rounded to the nearest odd number to be used as the search window size. The search window size model is defined as:

$$
S=\operatorname{round}_{\text {odd }}(0.117 \sigma+9.758)
$$

where, $\operatorname{round}_{\text {odd }}()$ rounds to the nearest odd integer and $S \times S$ is the dimension of the search window.

\subsubsection{The two-step approach}

Using the patch and search window size models along with the exponential weight thresholding model, the proposed method was applied on the training image set as a multiple step approach. 
Table 3.7: PSNR comparison of proposed method for multiple steps for various noise levels

\begin{tabular}{|c|c|c|c|c|}
\hline Noise Level & 1 step & 2 step & 3 step & 4 step \\
\hline 10 & 34.11 & $\mathbf{3 4 . 1 6}$ & 33.71 & 33.56 \\
\hline 20 & 32.43 & $\mathbf{3 2 . 7 0}$ & 32.31 & 32.23 \\
\hline 30 & 29.55 & $\mathbf{2 9 . 8 6}$ & 29.47 & 29.34 \\
\hline 40 & 27.78 & $\mathbf{2 8 . 2 9}$ & 27.92 & 27.83 \\
\hline 50 & 25.44 & $\mathbf{2 6 . 1 0}$ & 25.77 & 25.69 \\
\hline 60 & 24.66 & $\mathbf{2 5 . 4 2}$ & 25.18 & 25.08 \\
\hline 70 & 23.99 & $\mathbf{2 4 . 8 8}$ & 24.73 & 24.66 \\
\hline 80 & 23.61 & $\mathbf{2 4 . 6 2}$ & 24.48 & 24.30 \\
\hline 90 & 22.79 & 23.96 & $\mathbf{2 3 . 9 9}$ & 23.85 \\
\hline 100 & 22.48 & 23.76 & $\mathbf{2 3 . 8 0}$ & 23.68 \\
\hline Average & 26.68 & $\mathbf{2 7 . 3 7}$ & 27.14 & 27.02 \\
\hline
\end{tabular}

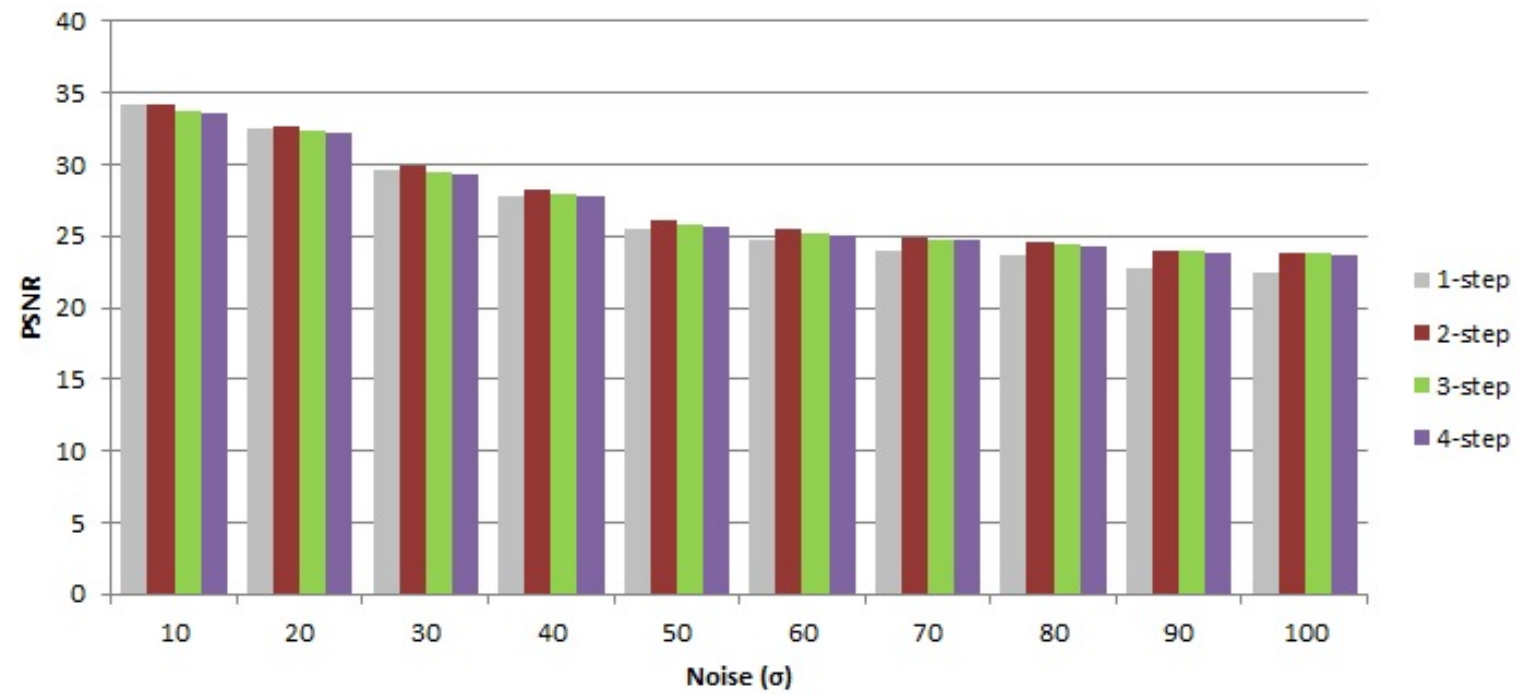

Figure 3.10: Bar chart of PSNR comparison of proposed method for multiple steps

In each step, the proposed method was applied on the output image of the previous step. After each iteration, the PSNR of the resulting denoised image was measured. The change in the PSNR measurement, compared to the previous iteration, was used to determine the number of iterations which demonstrated satisfactory performance improvement due to the extra iteration. The PSNR comparison of multiple iterations of the proposed method is shown in Table 3.7. The 2-step has the optimal performance compared to the other multi-step approaches. 


\subsection{Summary of the selected parameters}

The key parameters of the proposed method were empirically defined using different models.

The percentile of cut-off weight used for thresholding is defined as:

$$
w_{\text {percentile }}=\operatorname{ceil}\left(100 \times e^{-0.01 a \sigma}\right)
$$

The patch-size, $P \times P$, is selected as shown below:

$$
P= \begin{cases}7 & \text { if } \sigma<85 \\ 9, & \text { otherwise }\end{cases}
$$

The search window size, $S \times S$ is determined as:

$$
S=\operatorname{round}_{o d d}(0.117 \sigma+9.758)
$$




\section{Chapter 4}

\section{Experimental Results and Analysis}

The proposed image denoising method has been tested on the standard Kodak image set. The images are corrupted with additive white Gaussian noise with the noise level (standard deviation), $\sigma$ ranging from 10 to 100 , with step size equals 10 . The proposed method is compared with the original non-local means scheme, variants of non-local means and the BM3D method.

\subsection{Image set}

All the experiments are carried out of the standard Kodak gray-scale image set. It comprises of 24 gray-scale images of dimensions $768 \times 512$ and $512 \times 768$. The images in the Kodak image set are shown in Figure 4.1. The standard images are noise-free and additive white Gaussian noise is added on top of the noise-free image to generate the noisy image.

\subsection{Noise Generation}

The additive noise signal is added to the original noise-free signal in order to generate the noisy signal. For the purpose of our experimentation, the standard noise-free image are contaminated by adding Gaussian white noise, distributed throughout the image. The final intensity values 


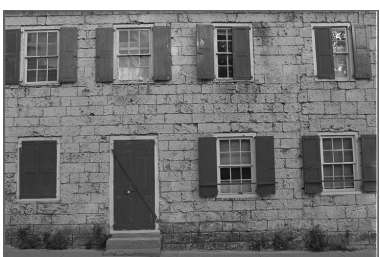

(a) Windows

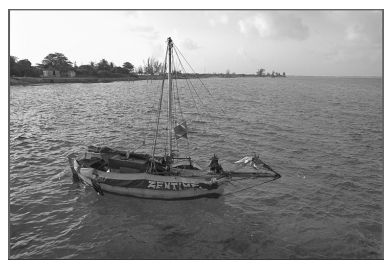

(e) Lake

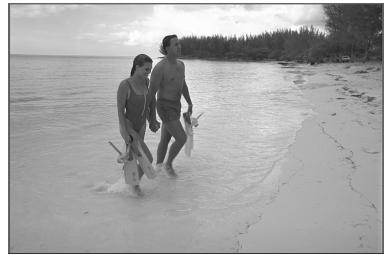

(i) Beach

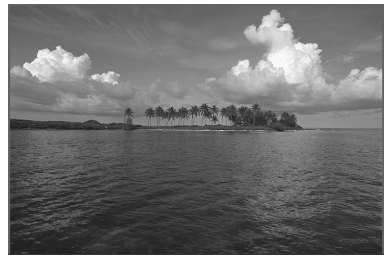

(m) Island

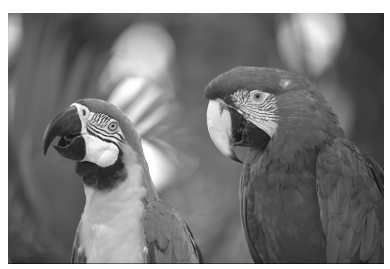

(q) Parrot

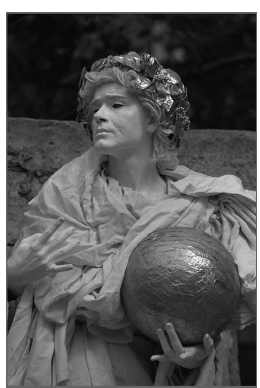

(u) Statue

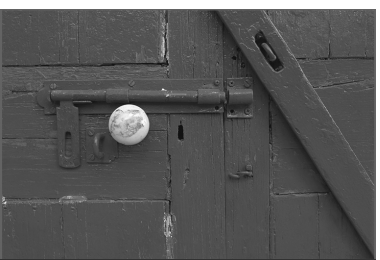

(b) Door

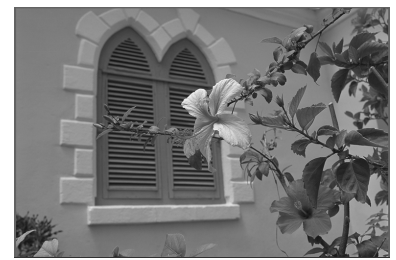

(f) Flower

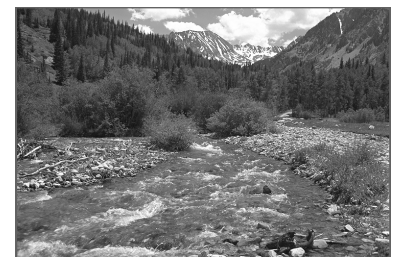

(j) Landscape

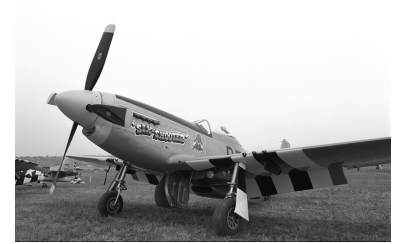

(n) Plane

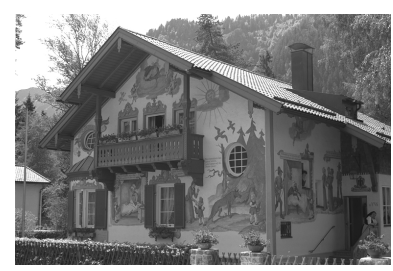

(r) House 2

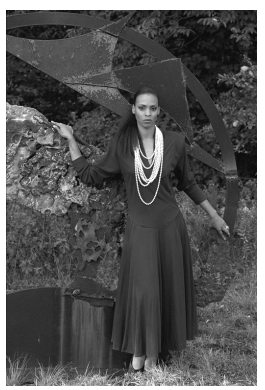

(v) Model

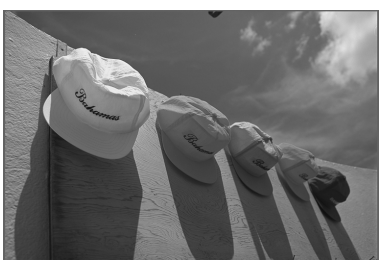

(c) Hats

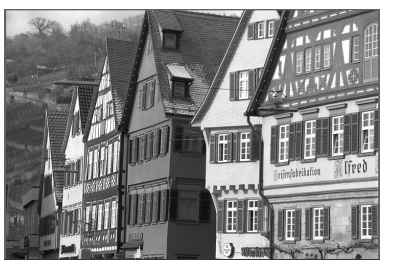

(g) Houses

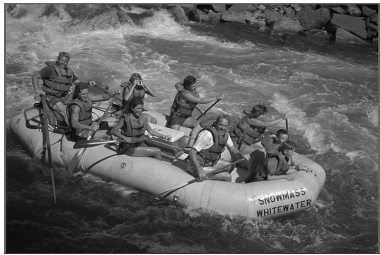

(k) Raft

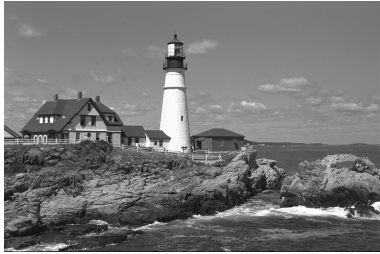

(o) Lighthouse

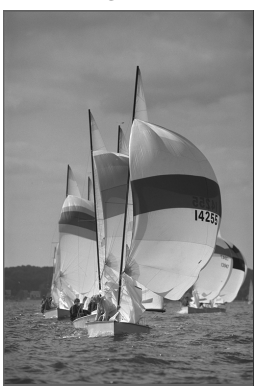

(s) Sail

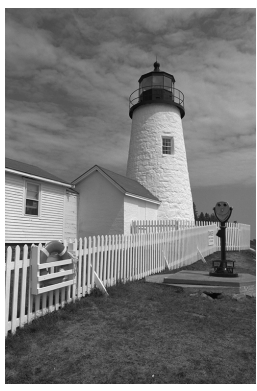

(w) Lighthouse 2

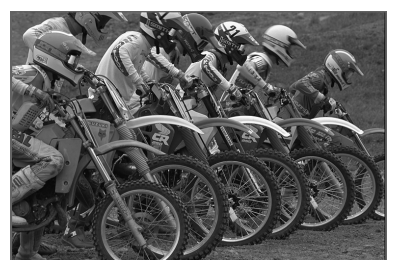

(d) Bike

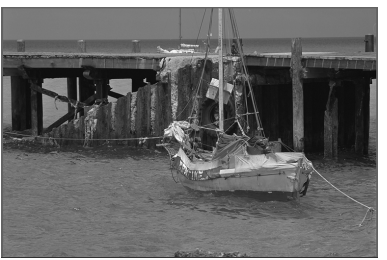

(h) Bridge

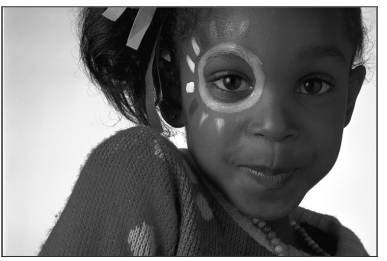

(l) Girl

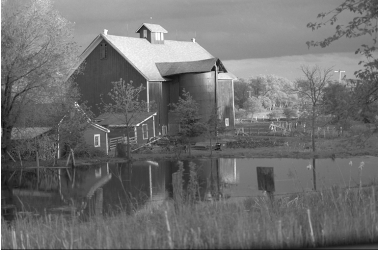

(p) House

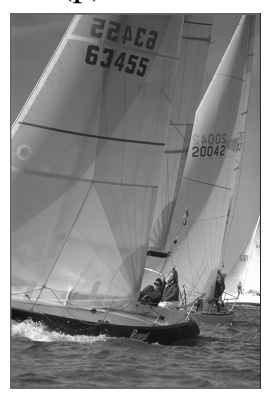

(t) Boat 2

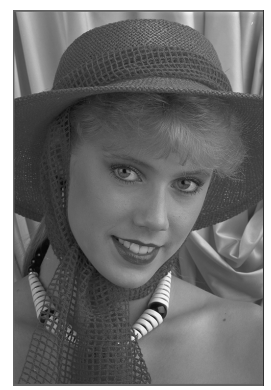

(x) Woman

Figure 4.1: Kodak Image Set (Grayscale) 


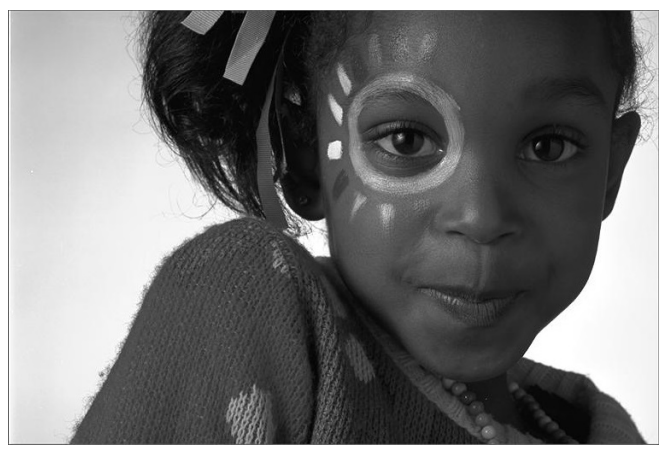

(a) Original Image

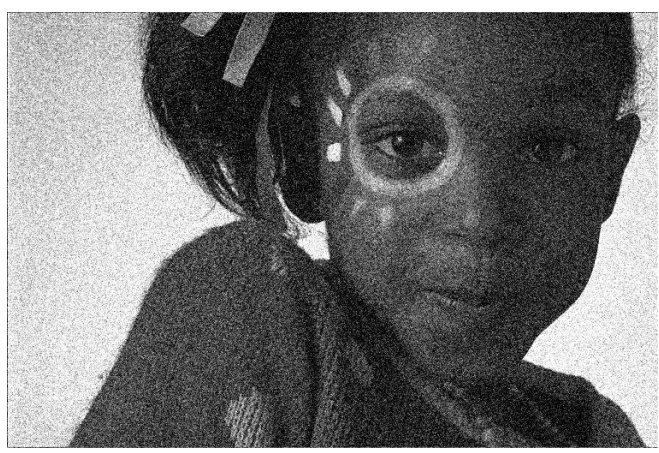

(b) Noisy Image

Figure 4.2: Example of Gaussian noise $(\sigma=40)$ added to a noise-free image

were kept within the maximum intensity value of gray-scale images. In our experiments, all the noisy images are generated in MATLAB using the following method:

$$
\text { imNoise }=i m+\left(\frac{\sigma}{255} \times \operatorname{randn}(\operatorname{size}(\mathrm{im}))\right)
$$

where imNoise is the noisy image, im is the original, noise-free image, $\sigma$ is the required noise level and the function $\operatorname{randn}()$ generates a random matrix with Gaussian distribution and size equal to the dimension of the original input image.

An example of additive white Gaussian noise added to a noise-free image is shown in Figure 4.2

\subsection{Performance Measure}

To measure the performance of our proposed method in comparison to other existing denoising methods, we have used the Peak Signal to Noise Ratio (PSNR) and the Mean Structural SIMilarity (MSSIM) measure. These measures are generally used for objective evaluation and measurement of various denois- 
ing methods. We also performed subjective comparison between our proposed method and existing denoising methods.

\subsubsection{Peak Signal to Noise Ratio (PSNR)}

The Peak Signal to Noise Ratio measures the ratio between the maximum possible power of a signal to the power of the noise which affects the quality of the original signal. The PSNR is usually expressed as the logarithmic decibel scale. A higher value in PSNR represents better reconstructed or denoised image. The PSNR is measured using:

$$
P S N R=10 \log _{10}\left(\frac{M A X_{I}^{2}}{M S E}\right)
$$

where $M A X_{I}$ represents the maximum intensity of the image and $M S E$ measures the mean squared error between the original image and the degraded image, defined as:

$$
M S E=\frac{1}{M \times N} \sum_{i=0}^{M} \sum_{j=0}^{N}\left(u_{i j}-v_{i j}\right)^{2},
$$

where $u_{i j}$ is the original image, $v_{i j}$ is the degraded image and the size of the images is $M \times N$.

\subsubsection{Mean Structural Similarity (MSSIM)}

One of the drawbacks of the PSNR measure is that it relies on the mean square error for calculating the ratio. Mean squared error considers only the differ- 
ences between isolated data points. To evaluate the performance of a denoising method based on the degree of structural similarity between the original and the reconstructed image, the Structural SIMilarity (SSIM) measure can be used [27][28]. The SSIM measure provides a better assessment of an image restoration or denoising method. The SSIM between two blocks is defined as:

$$
S S I M=\frac{\left(2 \mu_{x} \mu_{y}+c_{1}\right)\left(2 \sigma_{x y}+c_{2}\right)}{\left(\mu_{x}^{2}+\mu_{y}^{2}+c_{1}\right)\left(\sigma_{x}^{2}+\sigma_{y}^{2}+c_{2}\right.},
$$

where, $x$ and $y$ are two identical sized window or patch, $\mu_{x}$ and $\mu_{y}$ are the averages of $x$ and $y, \sigma_{x}^{2}$ and $\sigma_{y}^{2}$ are the variance of $x$ and $y$ and $\sigma_{x y}$ is the covariance. The mean SSIM (MSSIM), averaged over all SSIM, is used as for the quality measurement of a denoising method. 


\subsection{Results and Analysis}

The proposed method is compared with the original Non-Local Means (NLM) scheme, the Two-Stage Non-Local Means (TS-NLM) scheme, the Non-Local Euclidean Median (NLEM) scheme and the Block Matching and 3D Filtering (BM3D) scheme. All the objective measurements reported are averaged after repeating each of the experiments 10 times. The denoising performances are compared after running each of the denoising methods, including the proposed method, on the same noisy image for each noise level, $\sigma$.

\subsubsection{Performance evaluation using PSNR}

Table 4.1 shows the PSNR comparison for the Girl image and Table 4.2 shows the average PSNR values over all images in the Kodak image set, for various noise levels. Figure 4.3 shows the bar chart of PSNR comparison among the proposed method and existing denoising methods, for various noise levels. The PSNR values are reported after averaging the results of 10 runs. The standard deviation of PSNR for each of the noise levels is shown in Table 4.3.

The performance of the proposed method is better than the original nonlocal means method and its variant for all noise levels. Yet, when compared to $\mathrm{BM} 3 \mathrm{D}$, our proposed method managed to produce better results only when $\sigma \leq 80$. The proposed method also demonstrated better performance than existing methods on the average of all the noise levels used in our experiments. 
Table 4.1: PSNR comparison of the Girl image among the proposed method, the NLM method, variants of NLM and BM3D denoising scheme for various noise levels

\begin{tabular}{|c|c|c|c|c|c|}
\hline Noise Level & NLM & TS-NLM & NLEM & BM3D & Proposed \\
\hline 10 & 33.92 & 33.93 & 32.90 & 35.42 & $\mathbf{3 5 . 6 1}$ \\
\hline 20 & 31.83 & 32.01 & 31.85 & 33.46 & $\mathbf{3 3 . 6 0}$ \\
\hline 30 & 29.43 & 29.70 & 29.56 & 31.03 & $\mathbf{3 1 . 1 2}$ \\
\hline 40 & 28.47 & 28.96 & 28.61 & 29.88 & $\mathbf{3 0 . 0 4}$ \\
\hline 50 & 26.64 & 27.20 & 26.98 & 28.21 & $\mathbf{2 8 . 2 7}$ \\
\hline 60 & 25.12 & 25.77 & 25.30 & 26.53 & $\mathbf{2 6 . 6 0}$ \\
\hline 70 & 24.78 & 25.24 & 25.01 & 25.88 & $\mathbf{2 6 . 0 3}$ \\
\hline 80 & 23.69 & 24.46 & 23.93 & 25.33 & $\mathbf{2 5 . 5 0}$ \\
\hline 90 & 23.15 & 24.04 & 23.52 & $\mathbf{2 4 . 9 5}$ & 24.81 \\
\hline 100 & 22.91 & 23.88 & 23.18 & $\mathbf{2 4 . 4 3}$ & 24.28 \\
\hline Average & 26.99 & 27.52 & 27.08 & 28.51 & $\mathbf{2 8 . 5 9}$ \\
\hline
\end{tabular}

Table 4.2: PSNR comparison of the proposed method, the NLM method, variants of NLM and BM3D denoising scheme for various noise levels

\begin{tabular}{|c|c|c|c|c|c|}
\hline Noise Level & NLM & TS-NLM & NLEM & BM3D & Proposed \\
\hline 10 & 32.61 & 32.63 & 32.60 & 34.05 & $\mathbf{3 4 . 2 7}$ \\
\hline 20 & 30.77 & 30.94 & 30.82 & 32.25 & $\mathbf{3 2 . 4 3}$ \\
\hline 30 & 28.58 & 28.83 & 28.67 & 29.80 & $\mathbf{2 9 . 9 5}$ \\
\hline 40 & 27.02 & 27.47 & 27.29 & 28.19 & $\mathbf{2 8 . 3 3}$ \\
\hline 50 & 24.88 & 25.54 & 25.01 & 26.07 & $\mathbf{2 6 . 0 9}$ \\
\hline 60 & 23.93 & 24.66 & 24.16 & 25.38 & $\mathbf{2 5 . 4 6}$ \\
\hline 70 & 23.24 & 24.02 & 23.76 & 24.74 & $\mathbf{2 4 . 9 1}$ \\
\hline 80 & 22.90 & 23.56 & 23.23 & 24.46 & $\mathbf{2 4 . 6 5}$ \\
\hline 90 & 22.21 & 23.18 & 22.64 & $\mathbf{2 4 . 2 5}$ & 24.13 \\
\hline 100 & 21.98 & 22.83 & 22.29 & $\mathbf{2 3 . 9 7}$ & 23.84 \\
\hline Average & 25.81 & 26.36 & 26.05 & 27.32 & $\mathbf{2 7 . 4 1}$ \\
\hline
\end{tabular}




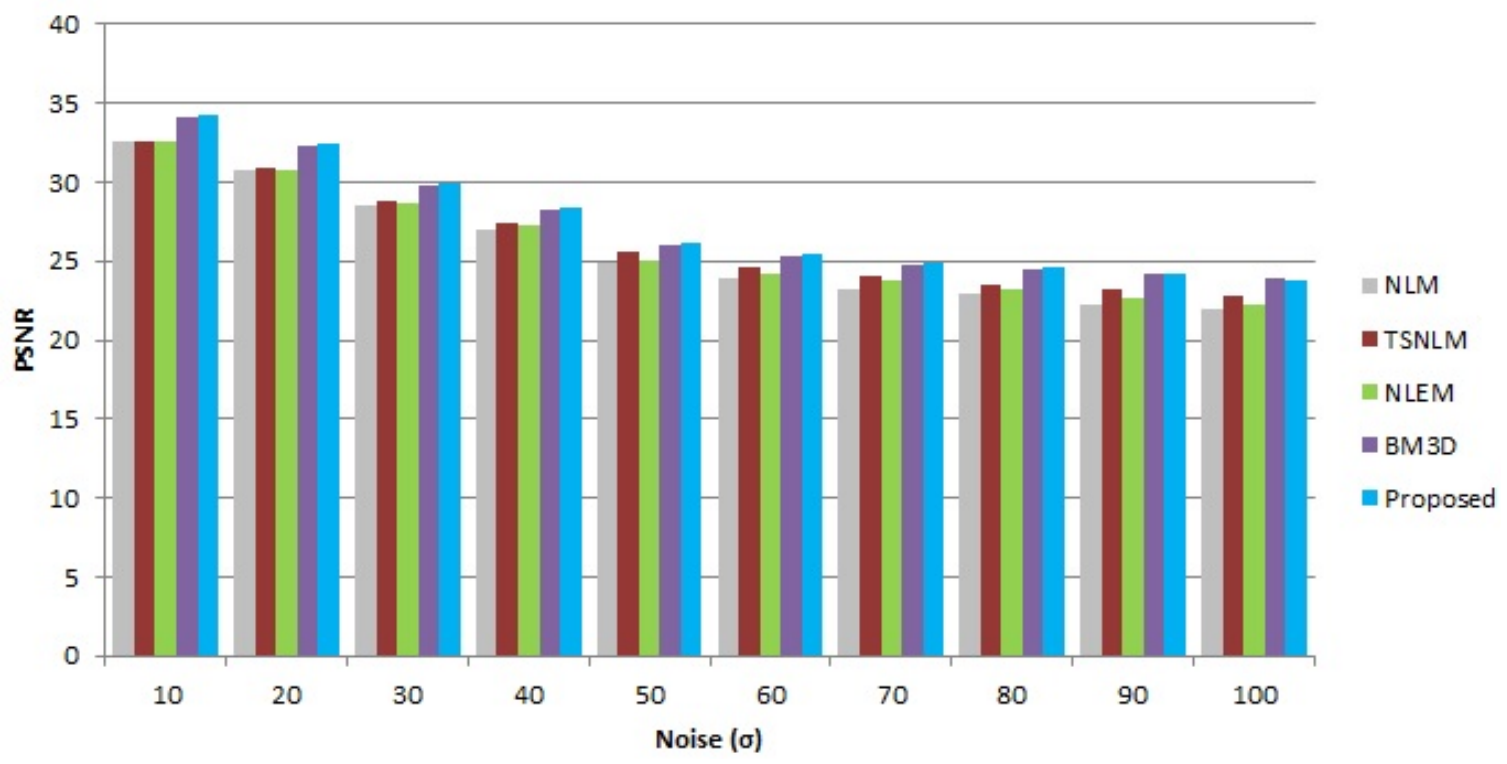

Figure 4.3: Bar chart of PSNR comparison of proposed and existing methods

Table 4.3: Standard deviation of PSNR values of proposed method and existing methods for different noise levels

\begin{tabular}{|c|c|c|c|c|c|}
\hline Noise Level & NLM & TS-NLM & NLEM & BM3D & Proposed \\
\hline 10 & 0.056 & 0.054 & 0.055 & 0.051 & 0.052 \\
\hline 20 & 0.063 & 0.061 & 0.062 & 0.059 & 0.058 \\
\hline 30 & 0.069 & 0.066 & 0.064 & 0.063 & 0.060 \\
\hline 40 & 0.084 & 0.082 & 0.078 & 0.076 & 0.077 \\
\hline 50 & 0.089 & 0.085 & 0.087 & 0.085 & 0.083 \\
\hline 60 & 0.094 & 0.096 & 0.096 & 0.095 & 0.096 \\
\hline 70 & 0.096 & 0.097 & 0.095 & 0.098 & 0.095 \\
\hline 80 & 0.102 & 0.100 & 0.101 & 0.102 & 0.103 \\
\hline 90 & 0.113 & 0.112 & 0.111 & 0.107 & 0.105 \\
\hline 100 & 0.115 & 0.114 & 0.113 & 0.111 & 0.112 \\
\hline Average & 0.088 & 0.087 & 0.086 & 0.085 & 0.084 \\
\hline
\end{tabular}




\subsubsection{Performance evaluation using MSSIM}

Table 4.4 shows the MSSIM comparison for the Girl image and Table 4.5 shows the MSSIM comparison over all images in the Kodak image set, for various noise levels. Figure 4.4 shows the bar chart of MSSIM comparison among the proposed method and existing denoising methods, for various noise levels. The MSSIM values are reported after averaging the results of 10 runs. The standard deviation of MSSIM for each of the noise levels is shown in Table 4.6.

In terms of MSSIM, the performance of the proposed method is consistent with PSNR, which means it is better than the original non-local means and its variant for all noise levels. When compared to BM3D, our proposed method managed to produce better results only when $\sigma \leq 80$. On average across all noise levels, the performance of proposed method has been found to be better than existing methods. 
Table 4.4: MSSIM comparison of the Girl image among the proposed method, the NLM method, variants of NLM and BM3D denoising scheme for various noise levels

\begin{tabular}{|c|c|c|c|c|c|}
\hline Noise Level & NLM & TS-NLM & NLEM & BM3D & Proposed \\
\hline 10 & 0.919 & 0.922 & 0.918 & 0.927 & $\mathbf{0 . 9 3 6}$ \\
\hline 20 & 0.875 & 0.882 & 0.878 & 0.889 & $\mathbf{0 . 8 9 7}$ \\
\hline 30 & 0.849 & 0.855 & 0.852 & 0.857 & $\mathbf{0 . 8 6 2}$ \\
\hline 40 & 0.818 & 0.822 & 0.819 & 0.832 & $\mathbf{0 . 8 3 4}$ \\
\hline 50 & 0.790 & 0.797 & 0.794 & 0.811 & $\mathbf{0 . 8 1 3}$ \\
\hline 60 & 0.761 & 0.767 & 0.763 & 0.780 & $\mathbf{0 . 7 8 4}$ \\
\hline 70 & 0.728 & 0.731 & 0.728 & 0.747 & $\mathbf{0 . 7 5 0}$ \\
\hline 80 & 0.713 & 0.717 & 0.715 & 0.738 & $\mathbf{0 . 7 4 1}$ \\
\hline 90 & 0.692 & 0.694 & 0.693 & $\mathbf{0 . 7 2 4}$ & 0.720 \\
\hline 100 & 0.678 & 0.683 & 0.679 & $\mathbf{0 . 7 1 3}$ & 0.708 \\
\hline Average & 0.782 & 0.787 & 0.784 & 0.802 & $\mathbf{0 . 8 0 4}$ \\
\hline
\end{tabular}

Table 4.5: MSSIM comparison of the proposed method, the NLM method, variants of NLM and BM3D denoising scheme for various noise levels

\begin{tabular}{|c|c|c|c|c|c|}
\hline Noise Level & NLM & TS-NLM & NLEM & BM3D & Proposed \\
\hline 10 & 0.916 & 0.918 & 0.914 & 0.921 & $\mathbf{0 . 9 3 2}$ \\
\hline 20 & 0.871 & 0.876 & 0.873 & 0.882 & $\mathbf{0 . 8 9 1}$ \\
\hline 30 & 0.843 & 0.847 & 0.844 & 0.851 & $\mathbf{0 . 8 5 7}$ \\
\hline 40 & 0.815 & 0.817 & 0.815 & 0.826 & $\mathbf{0 . 8 2 9}$ \\
\hline 50 & 0.786 & 0.792 & 0.787 & 0.801 & $\mathbf{0 . 8 0 6}$ \\
\hline 60 & 0.755 & 0.760 & 0.758 & 0.772 & $\mathbf{0 . 7 7 8}$ \\
\hline 70 & 0.724 & 0.726 & 0.724 & 0.742 & $\mathbf{0 . 7 4 4}$ \\
\hline 80 & 0.709 & 0.714 & 0.711 & 0.734 & $\mathbf{0 . 7 3 5}$ \\
\hline 90 & 0.689 & 0.691 & 0.690 & $\mathbf{0 . 7 1 2}$ & 0.709 \\
\hline 100 & 0.672 & 0.678 & 0.674 & $\mathbf{0 . 7 0 8}$ & 0.704 \\
\hline Average & 0.777 & 0.782 & 0.779 & 0.795 & $\mathbf{0 . 7 9 8}$ \\
\hline
\end{tabular}




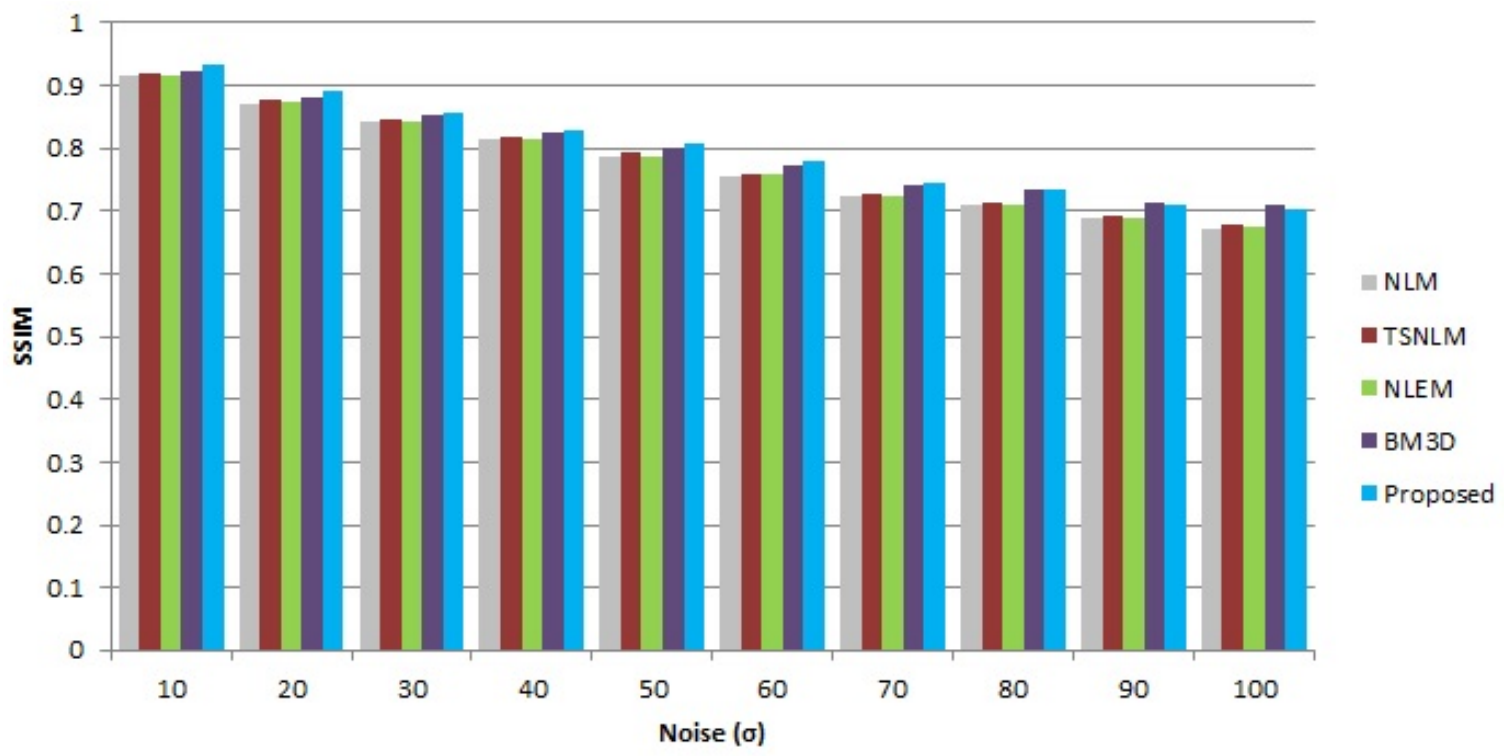

Figure 4.4: Bar chart of MSSIM comparison of proposed and existing methods

Table 4.6: Standard deviation of MSSIM values of proposed method and existing methods for different noise levels

\begin{tabular}{|c|c|c|c|c|c|}
\hline Noise Level & NLM & TS-NLM & NLEM & BM3D & Proposed \\
\hline 10 & 0.062 & 0.064 & 0.067 & 0.063 & 0.061 \\
\hline 20 & 0.075 & 0.074 & 0.071 & 0.072 & 0.068 \\
\hline 30 & 0.079 & 0.072 & 0.074 & 0.073 & 0.072 \\
\hline 40 & 0.082 & 0.084 & 0.087 & 0.085 & 0.084 \\
\hline 50 & 0.088 & 0.087 & 0.086 & 0.082 & 0.081 \\
\hline 60 & 0.092 & 0.094 & 0.097 & 0.092 & 0.094 \\
\hline 70 & 0.098 & 0.092 & 0.095 & 0.093 & 0.090 \\
\hline 80 & 0.099 & 0.095 & 0.099 & 0.097 & 0.098 \\
\hline 90 & 0.101 & 0.103 & 0.101 & 0.102 & 0.101 \\
\hline 100 & 0.108 & 0.104 & 0.107 & 0.105 & 0.103 \\
\hline Average & 0.088 & 0.087 & 0.088 & 0.086 & 0.085 \\
\hline
\end{tabular}




\subsubsection{Visual Comparison}

Along with the objective measure criteria for assessing the effectiveness of a image denoising method, it is also important to visually assess the quality of the denoised image. Figure 4.5 to 4.9 show the denoised image produced by the proposed method, the original NLM scheme, the variants of NLM and BM3D, on the Girl image. For noise level $\sigma=40$ and above, it can be observed that there are some visible noise artifacts remaining in the denoised image for the non-local means denosing scheme as well as its variants and BM3D. Figure 4.10 to Figure 4.14 shows the subjective comparison by zooming in on a particular region, the face. It can be observed that in the proposed method, the noise artifacts are better attenuated and there is less blurring of the face when compared to the non-local means method, the variants of non-local means and BM3D. 


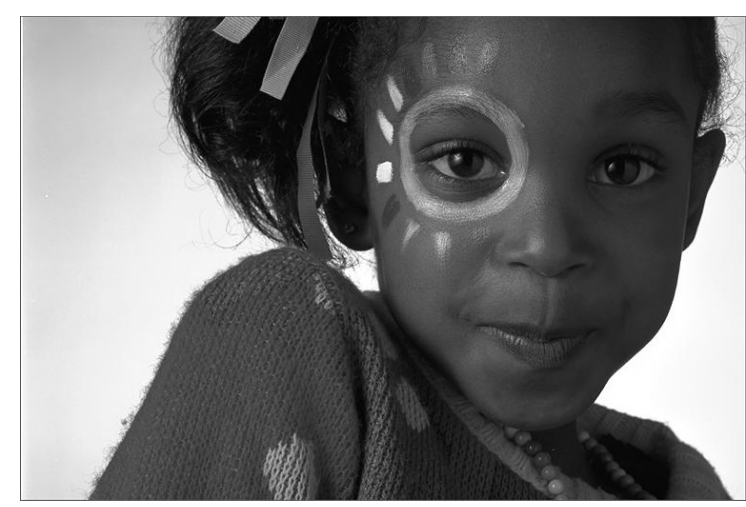

(a) Original

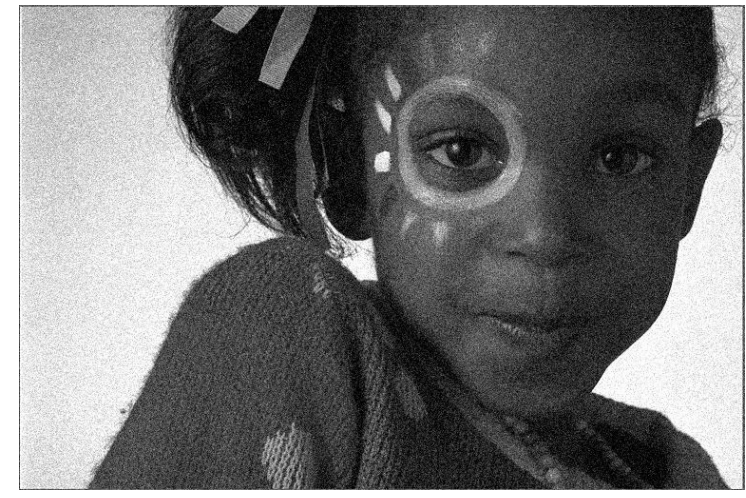

(b) Noise

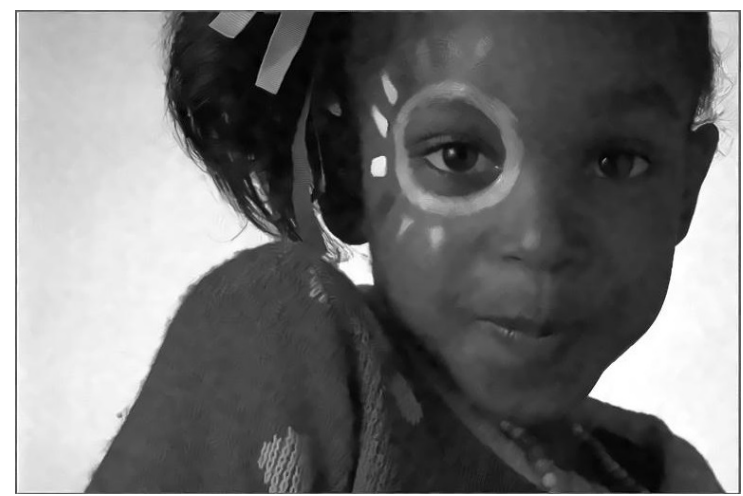

(d) TS-NLM

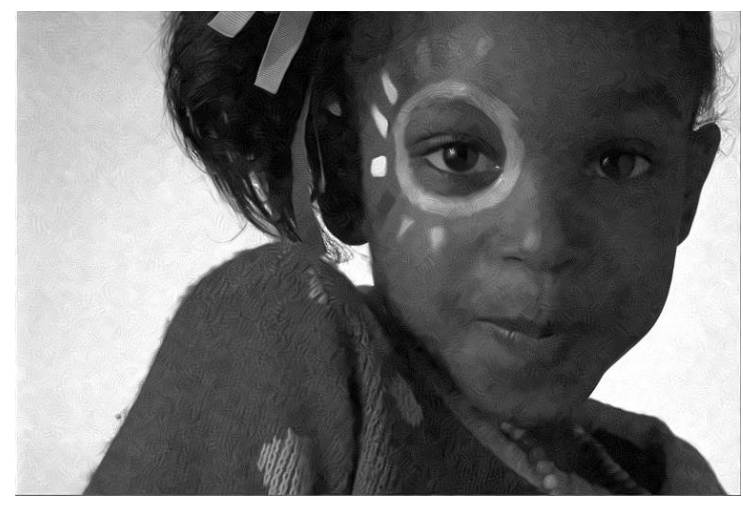

(f) BM3D

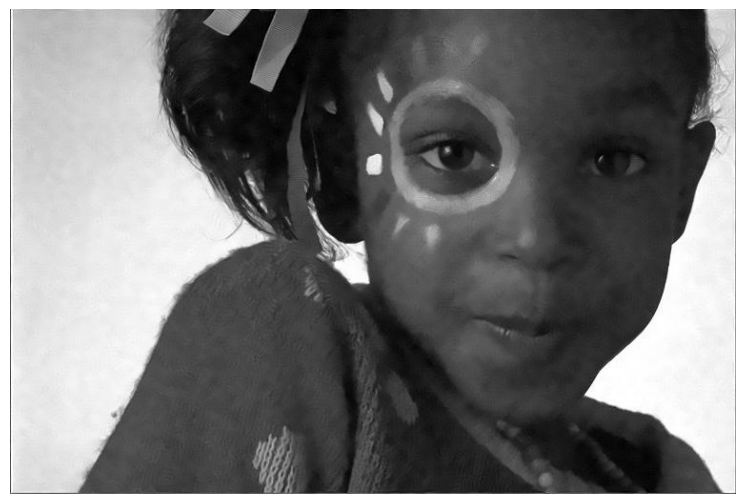

(c) NLM

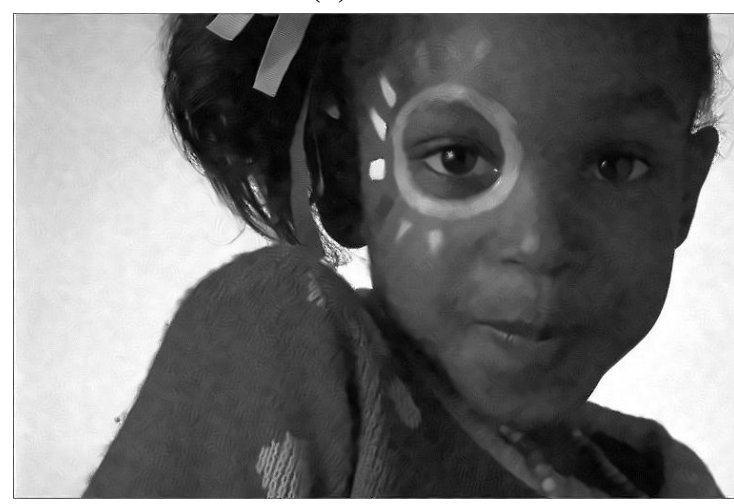

(e) NLEM

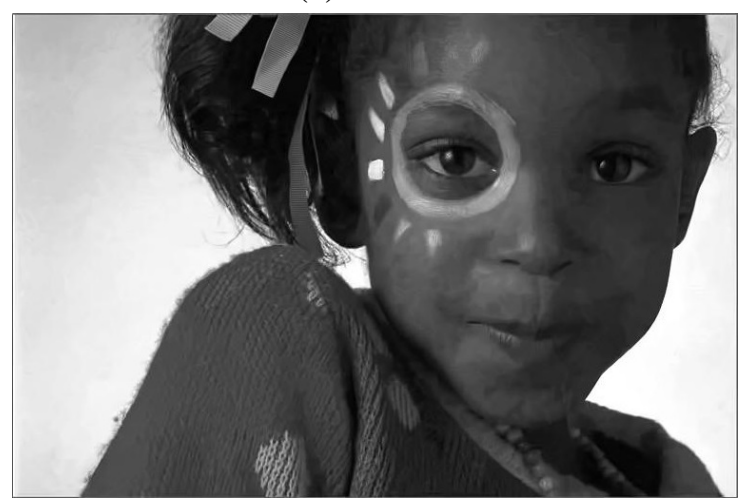

(g) Proposed

Figure 4.5: Visual Comparison for $\sigma=20$ 


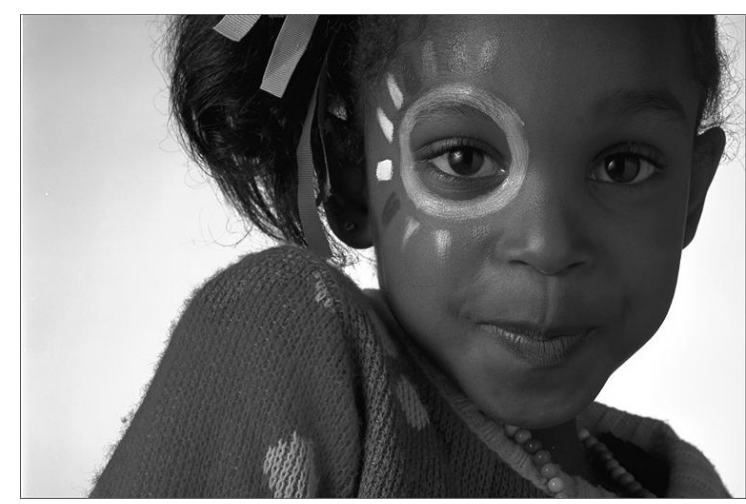

(a) Original

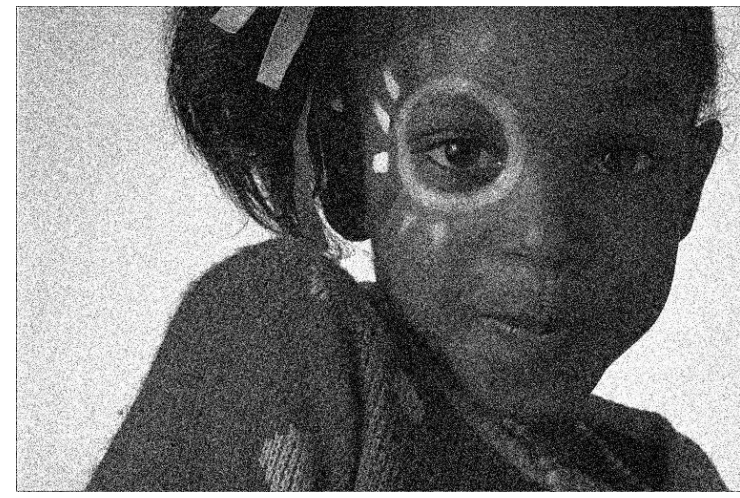

(b) Noise

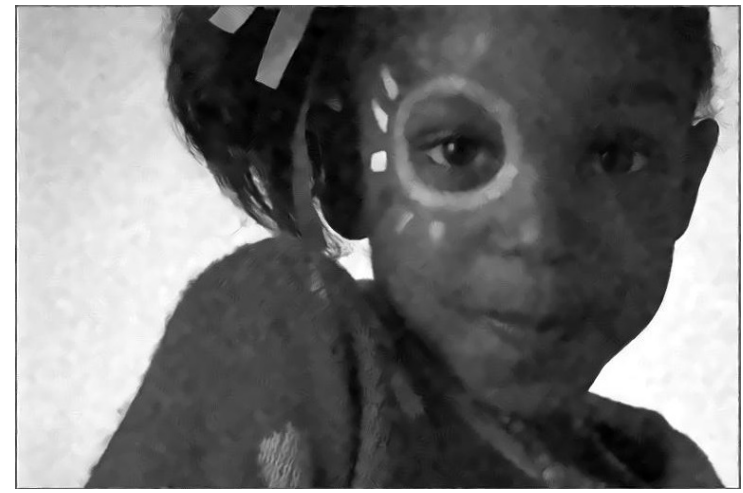

(d) TS-NLM

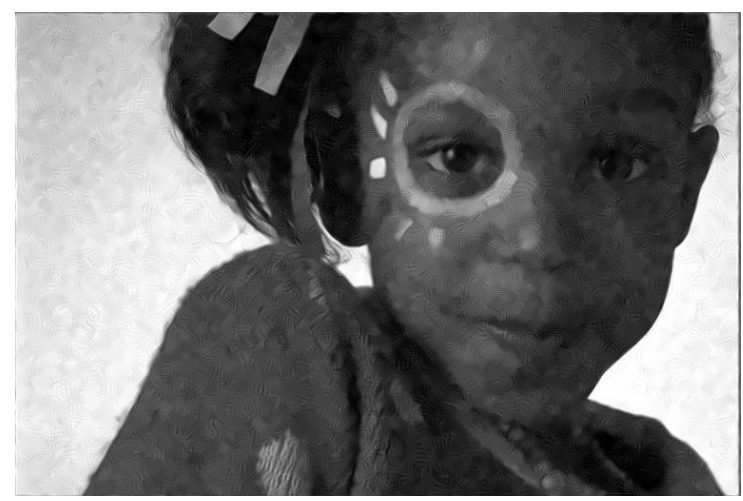

(f) BM3D

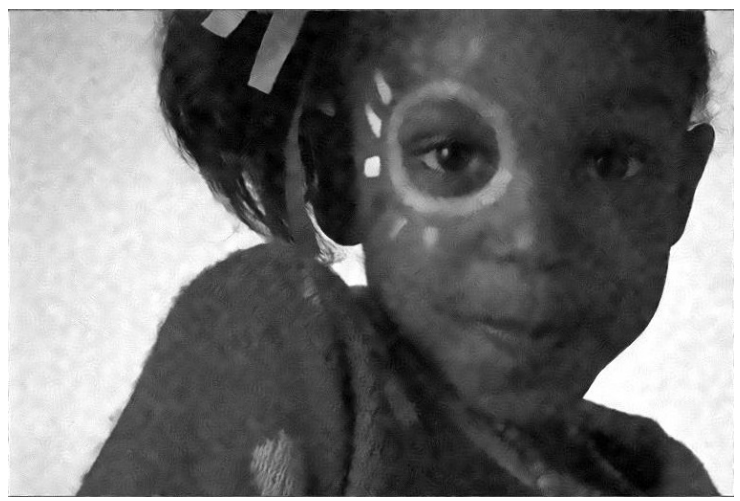

(c) NLM

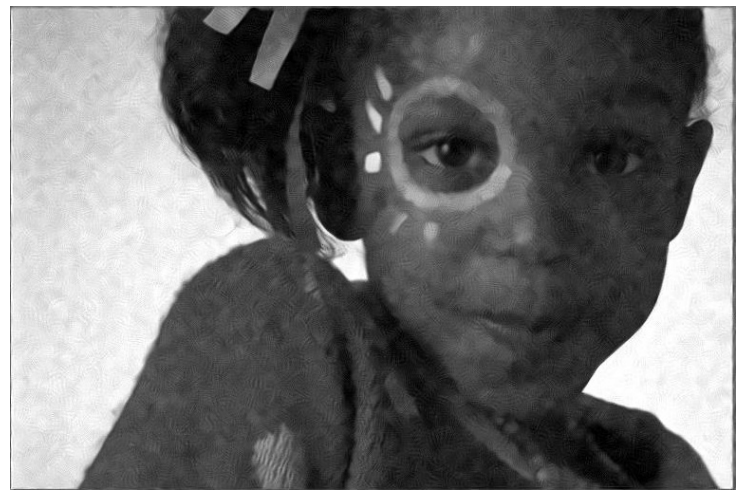

(e) NLEM

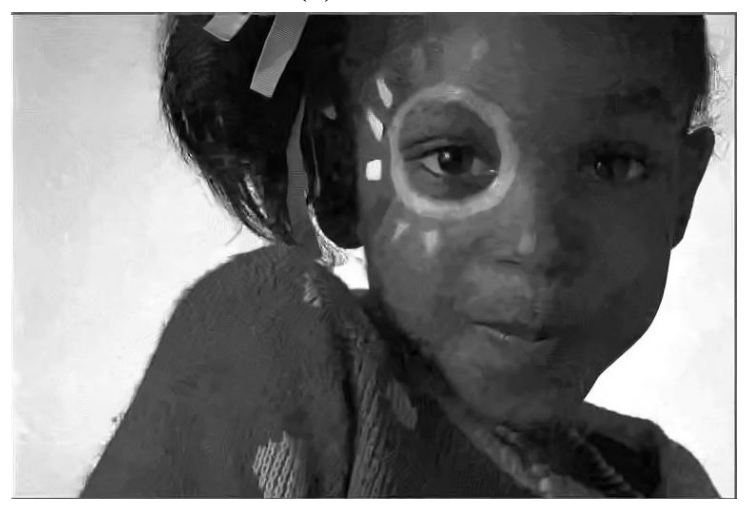

(g) Proposed

Figure 4.6: Visual Comparison for $\sigma=40$ 


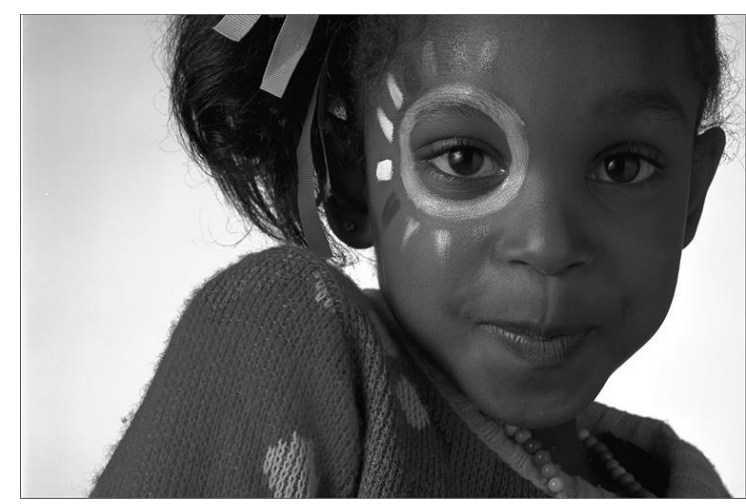

(a) Original

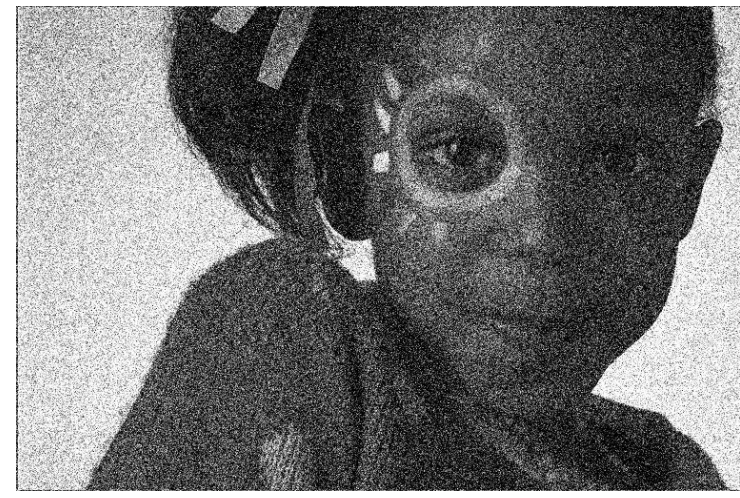

(b) Noise

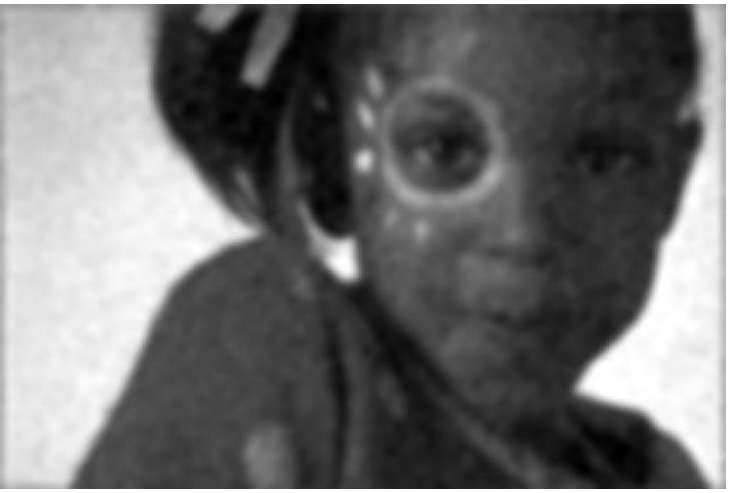

(d) TS-NLM

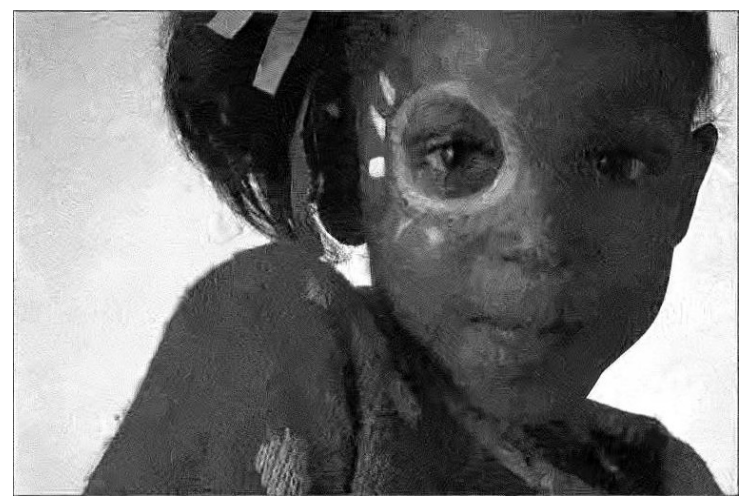

(f) BM3D

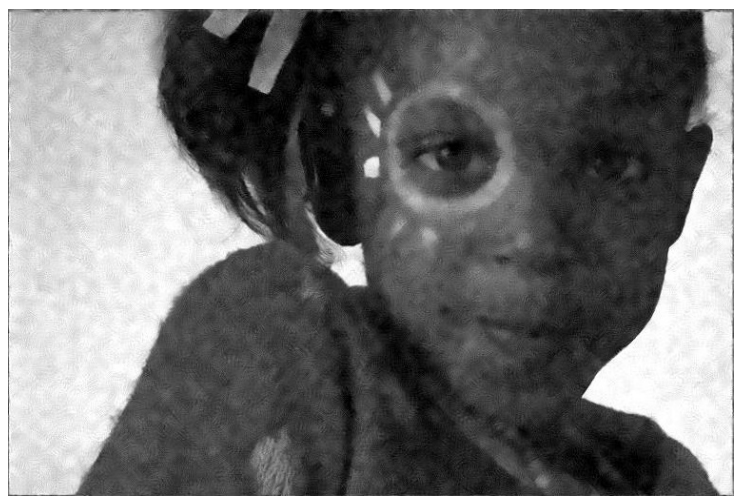

(c) NLM

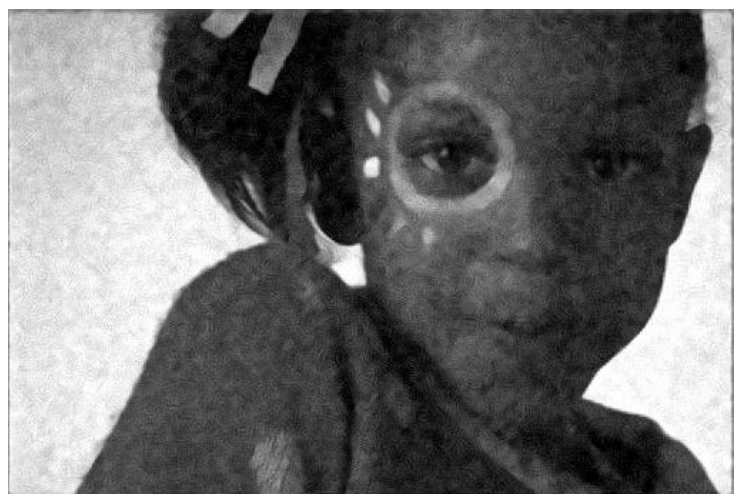

(e) NLEM

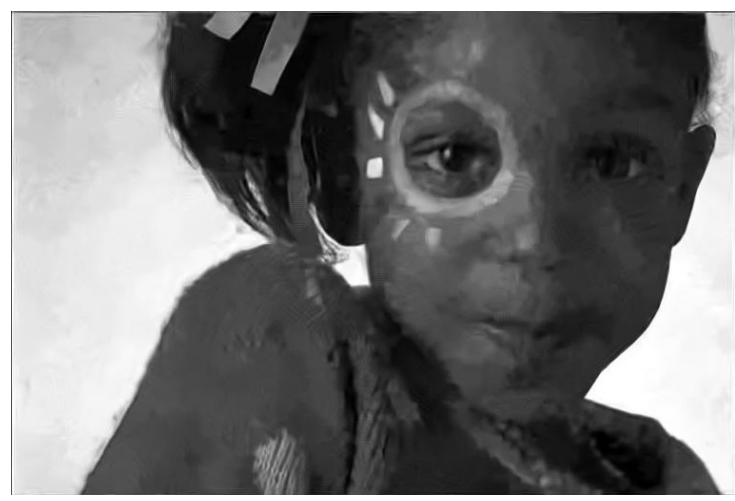

(g) Proposed

Figure 4.7: Visual Comparison for $\sigma=60$ 


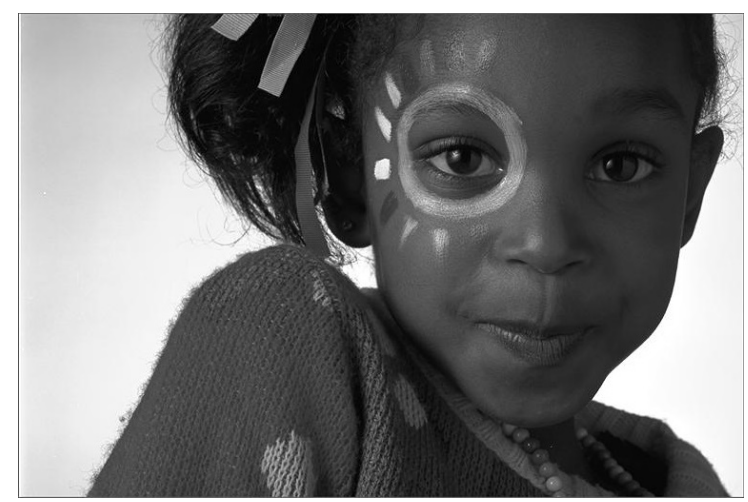

(a) Original

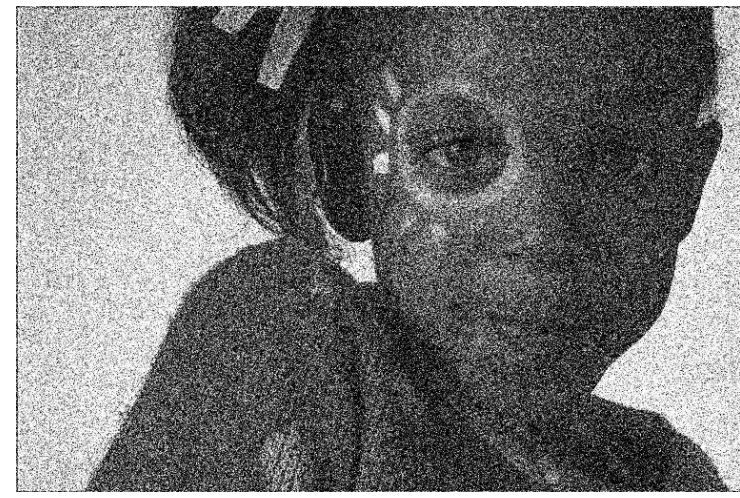

(b) Noise

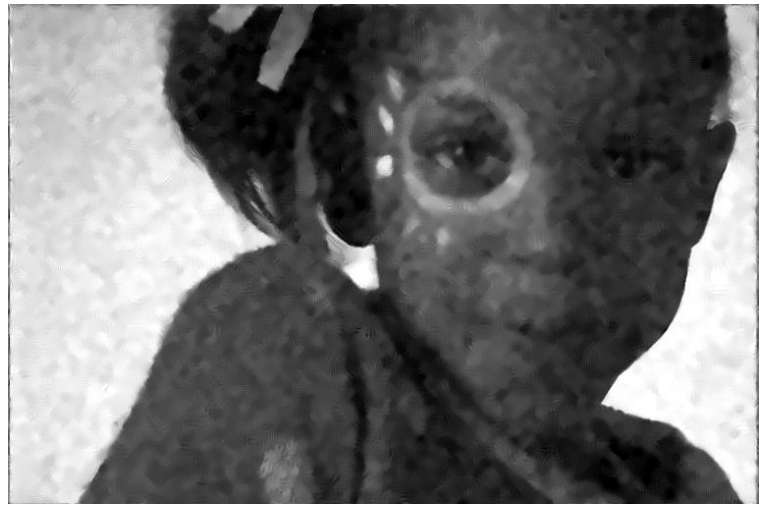

(d) TS-NLM

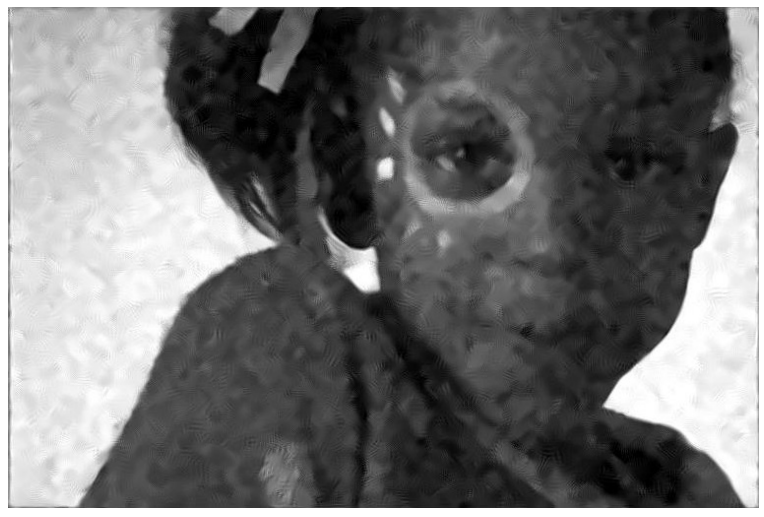

(f) BM3D

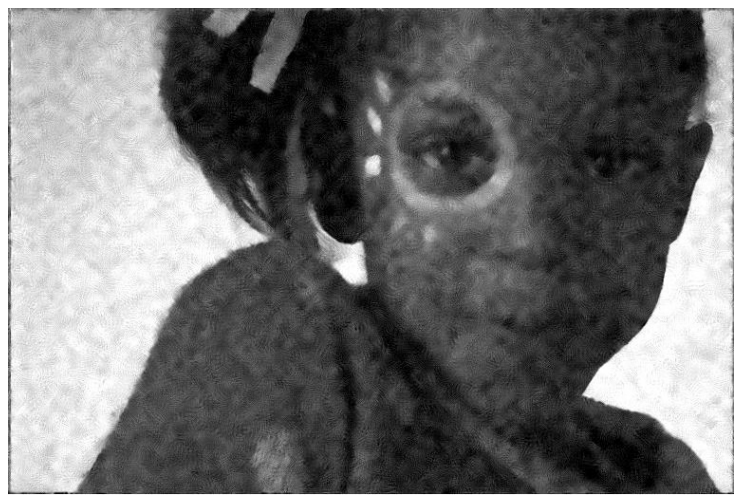

(c) NLM

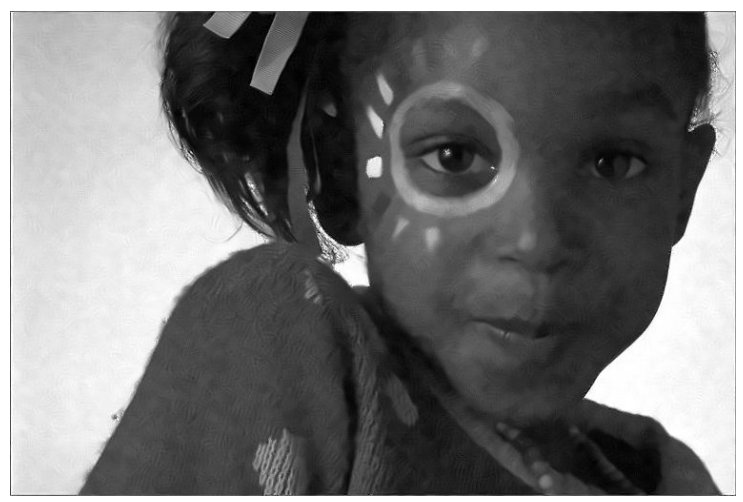

(e) NLEM

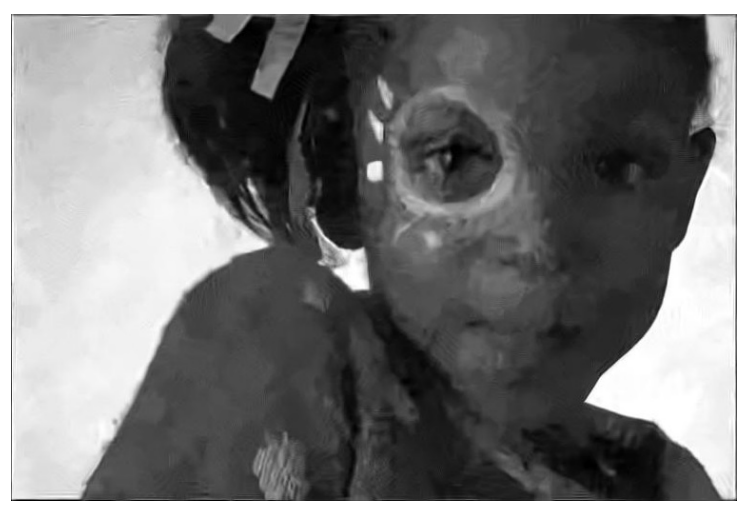

(g) Proposed

Figure 4.8: Visual Comparison for $\sigma=80$ 


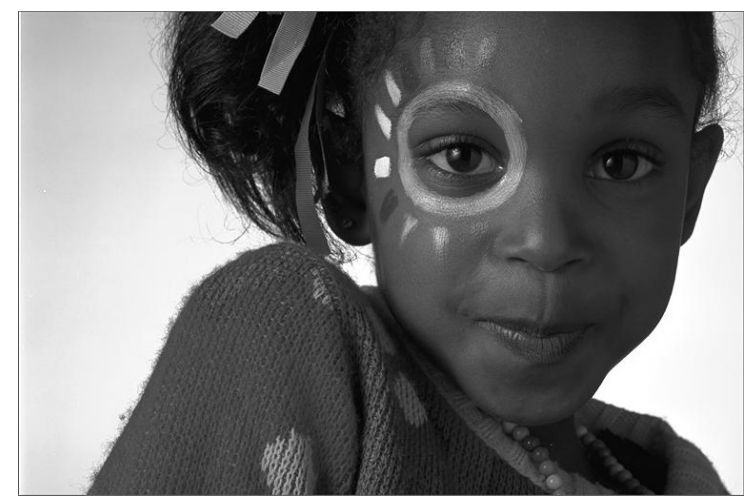

(a) Original

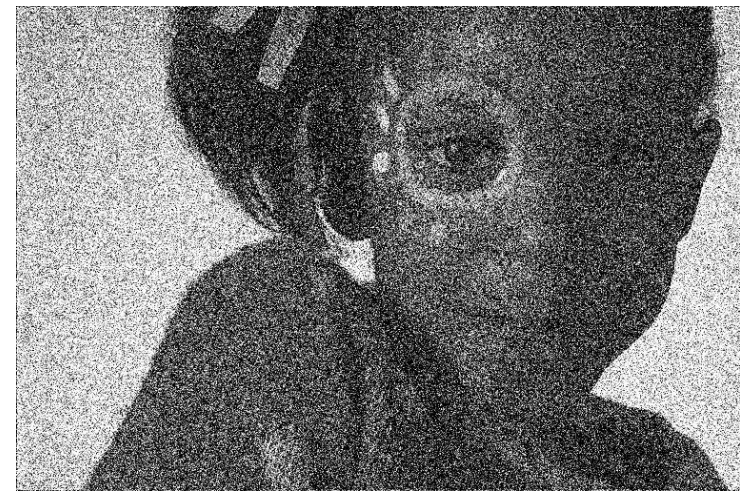

(b) Noise

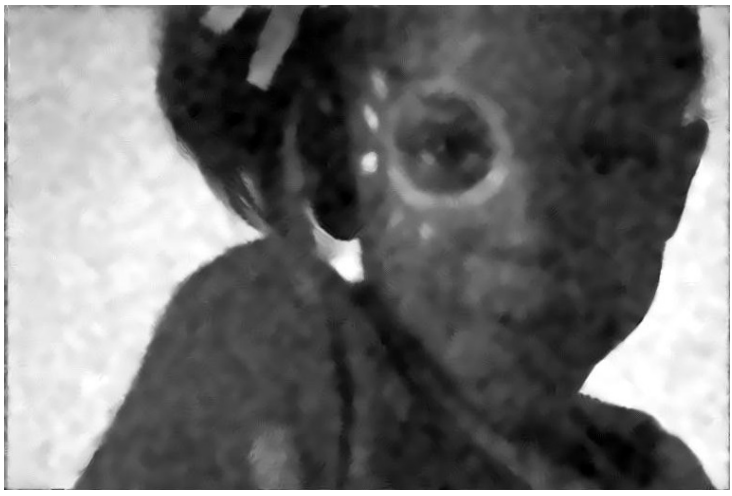

(d) TS-NLM

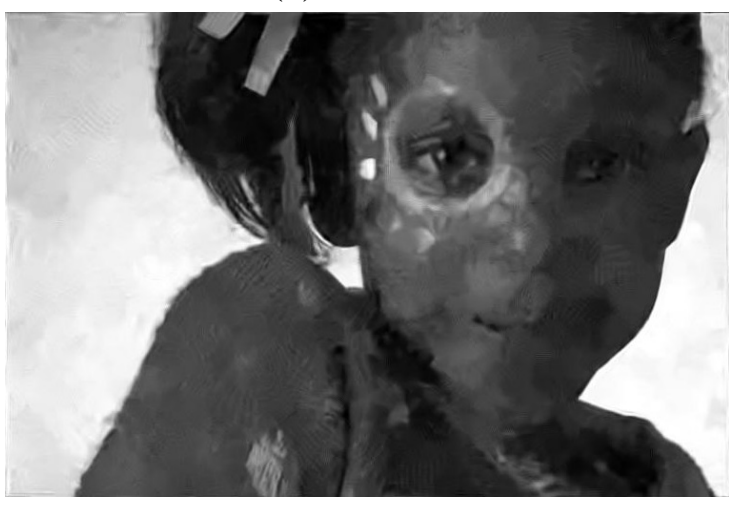

(f) BM3D

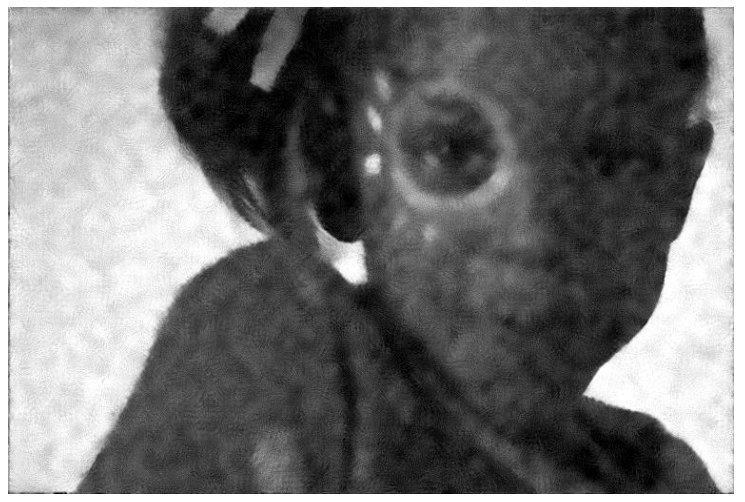

(c) NLM

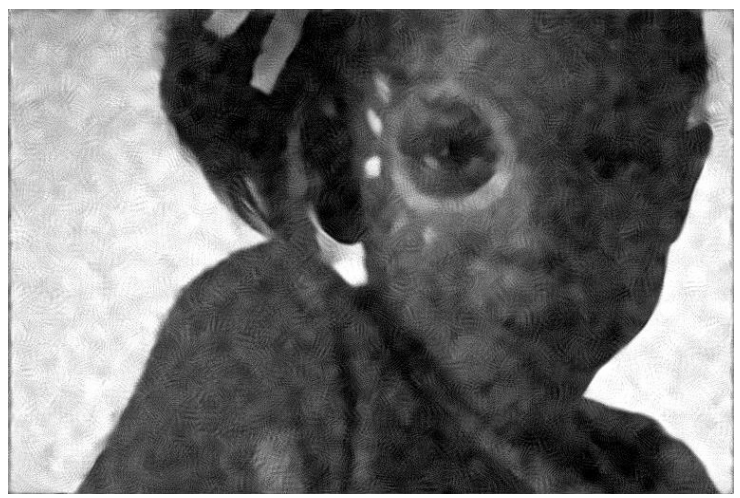

(e) NLEM

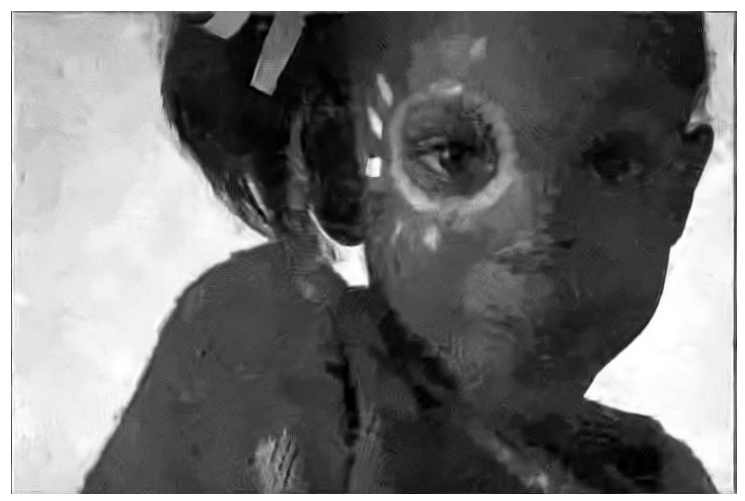

(g) Proposed

Figure 4.9: Visual Comparison for $\sigma=100$ 


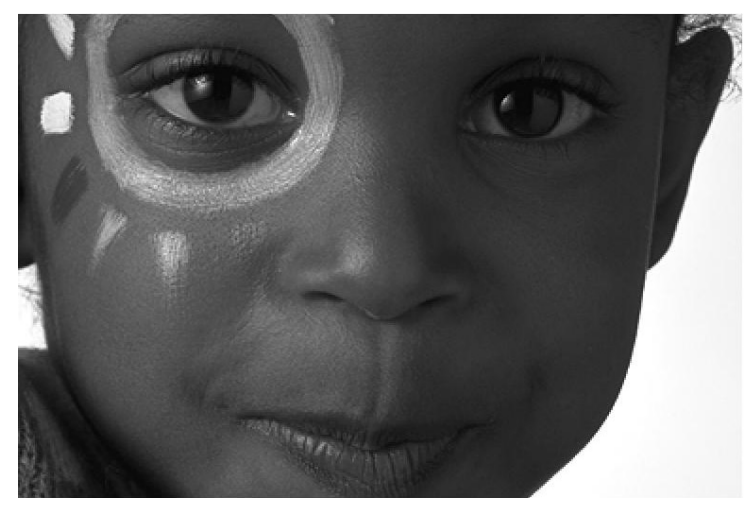

(a) Original

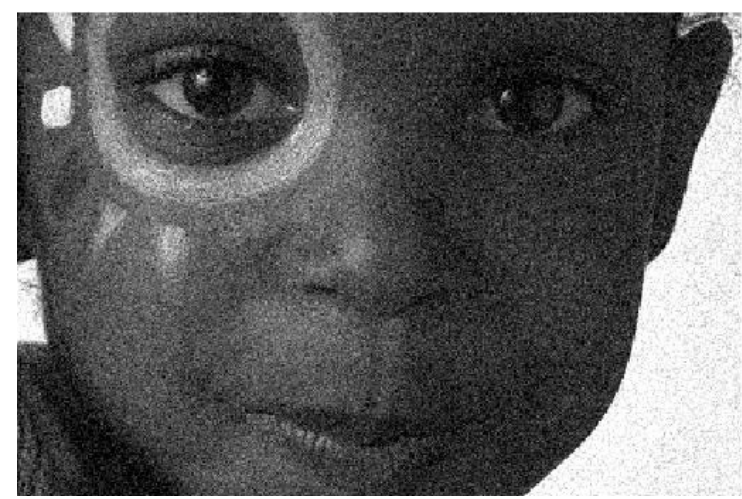

(b) Noise

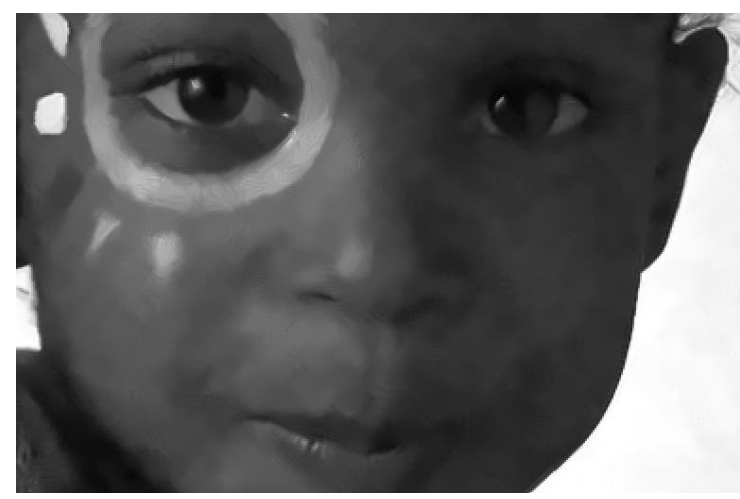

(d) TS-NLM

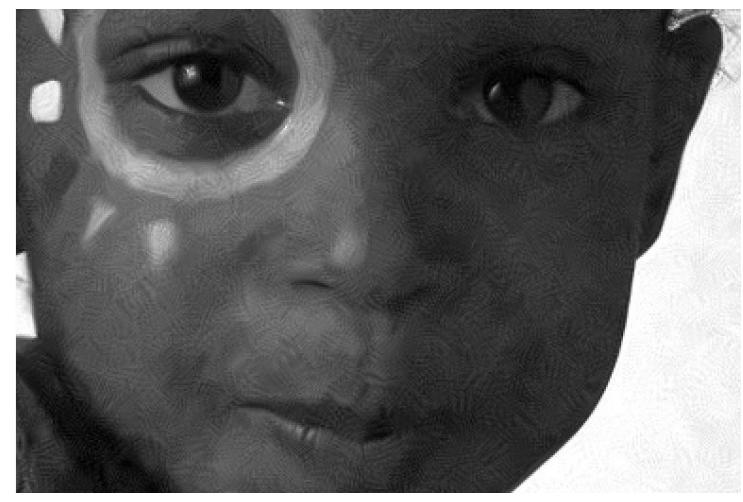

(f) BM3D

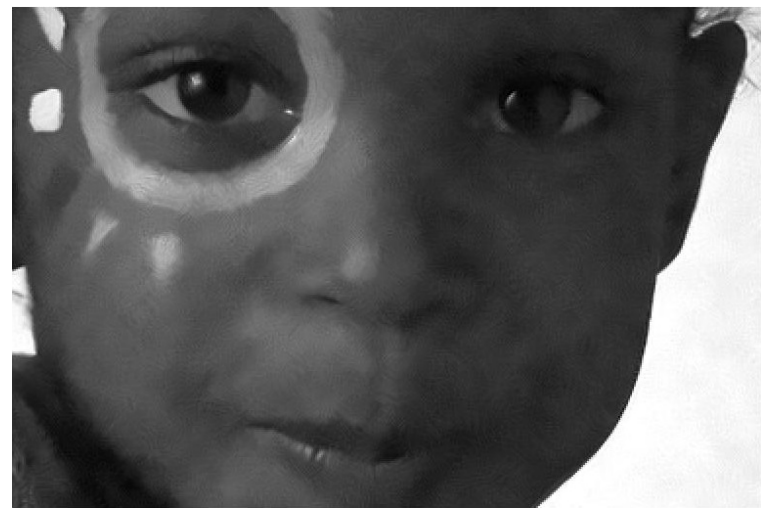

(c) NLM

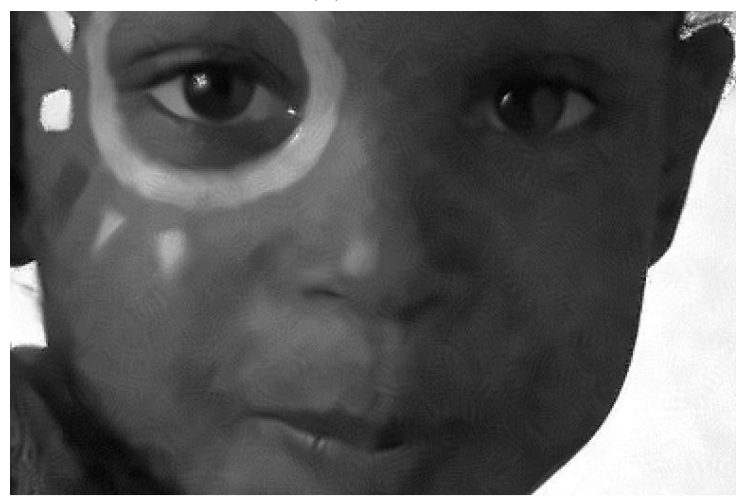

(e) NLEM

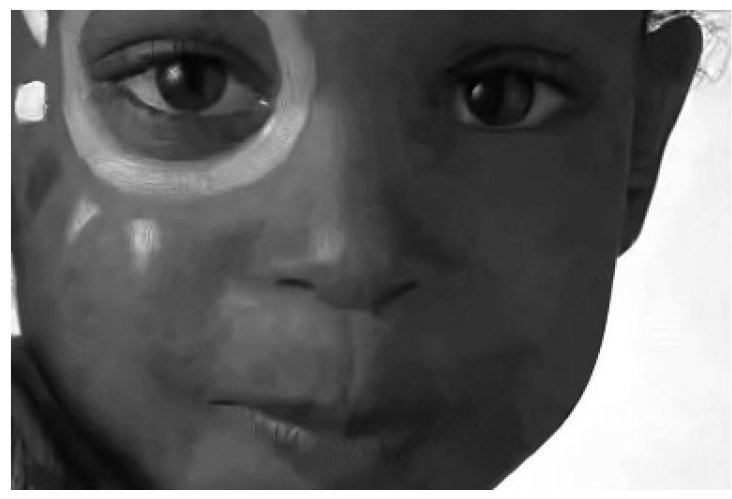

(g) Proposed

Figure 4.10: Visual Comparison (Zoom) for $\sigma=20$ 


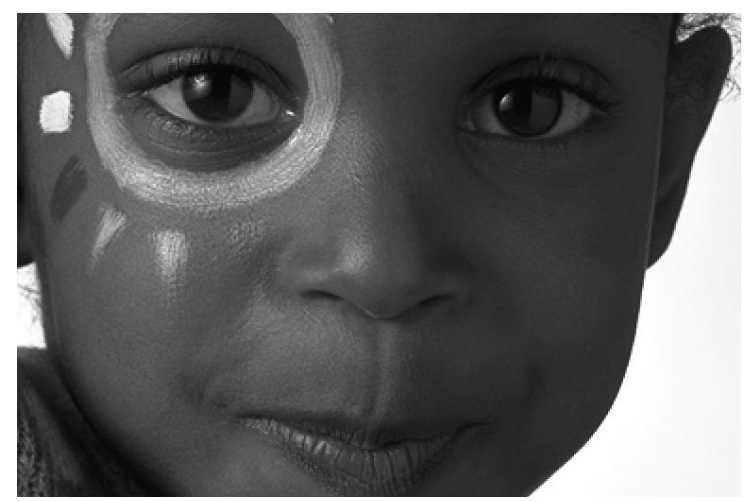

(a) Original

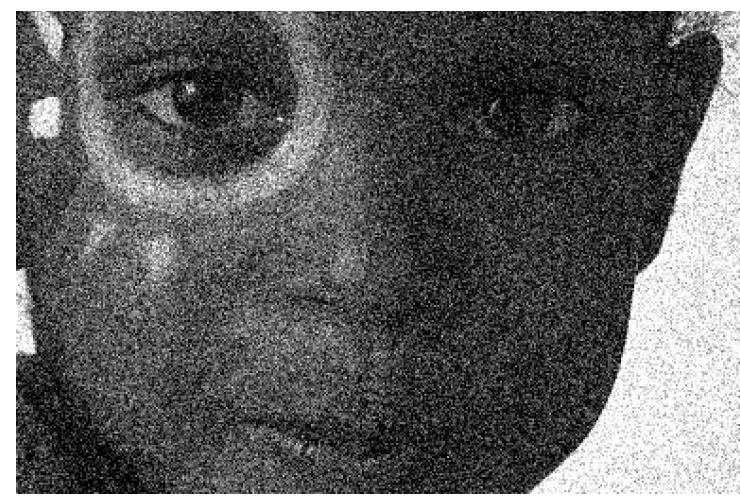

(b) Noise

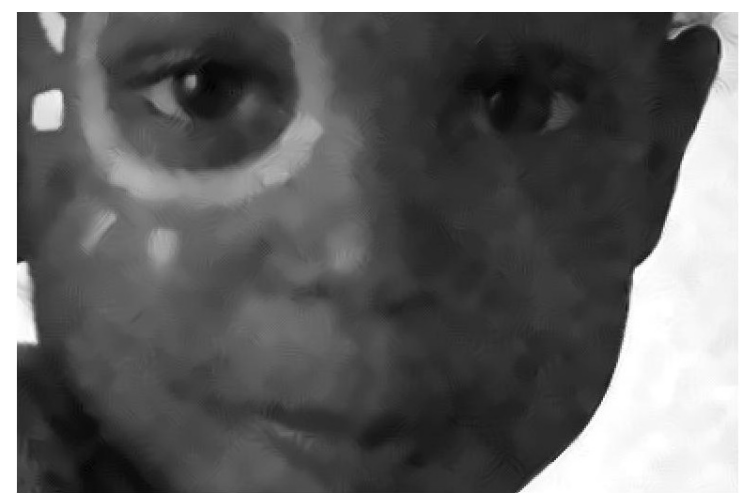

(d) TS-NLM

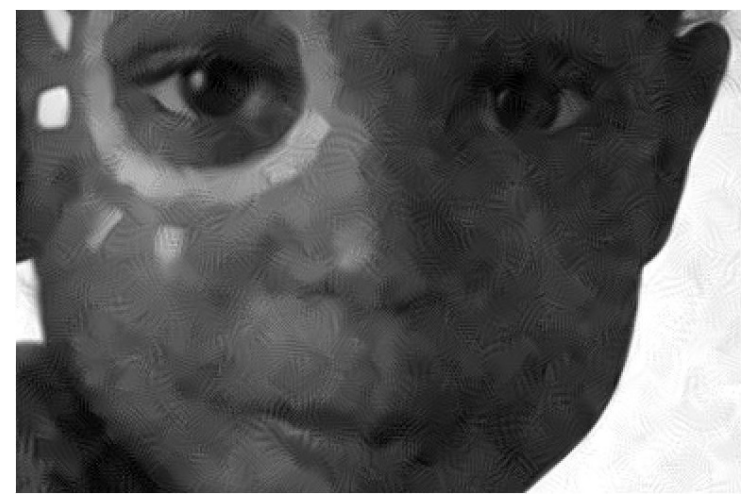

(f) BM3D

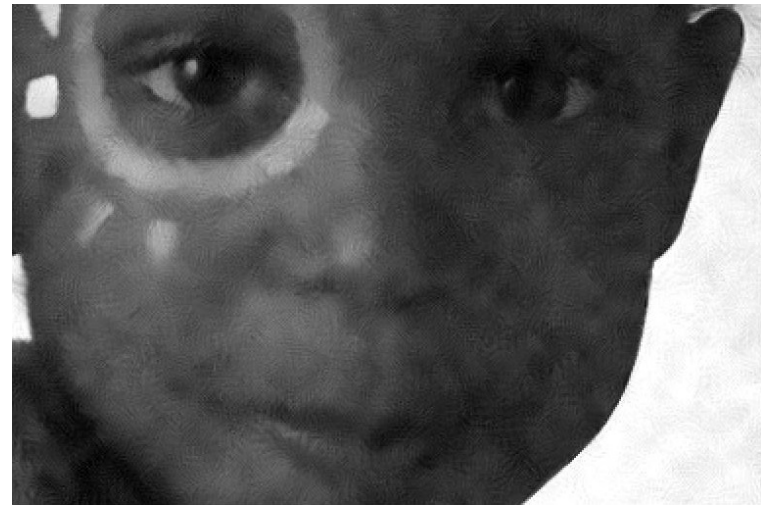

(c) NLM

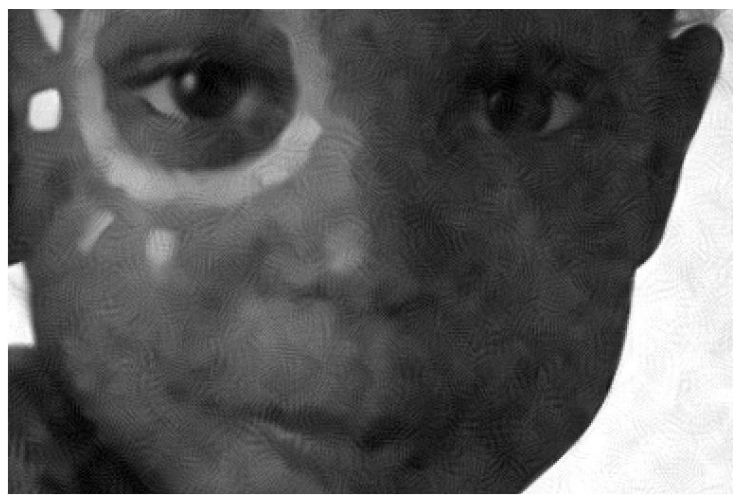

(e) NLEM

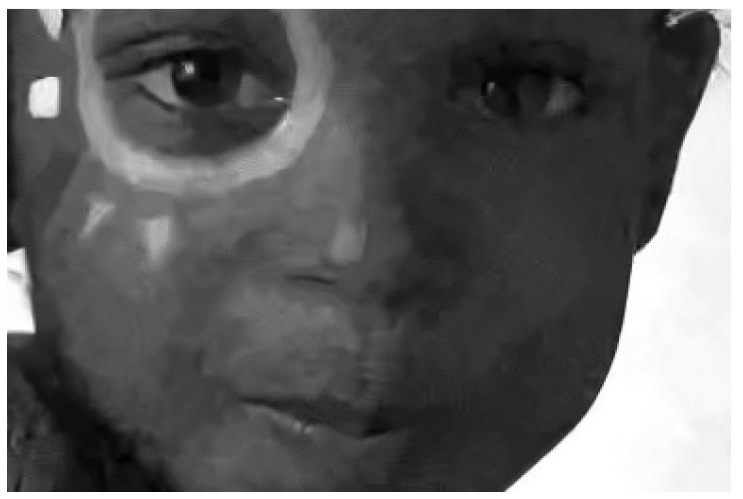

(g) Proposed

Figure 4.11: Visual Comparison (Zoom) for $\sigma=40$ 


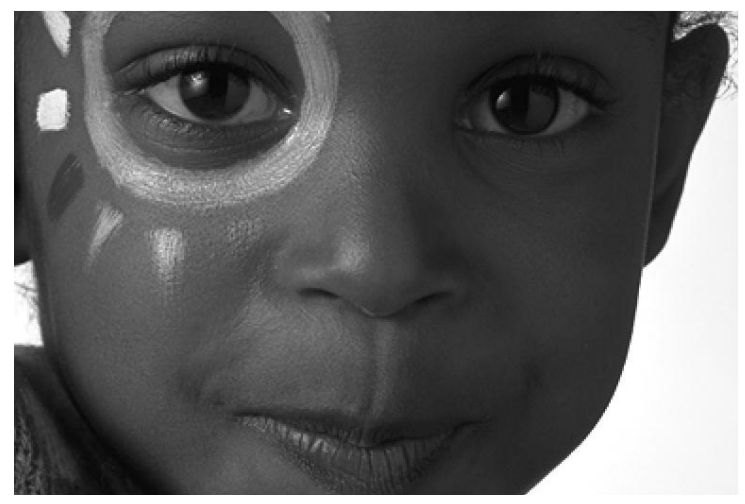

(a) Original
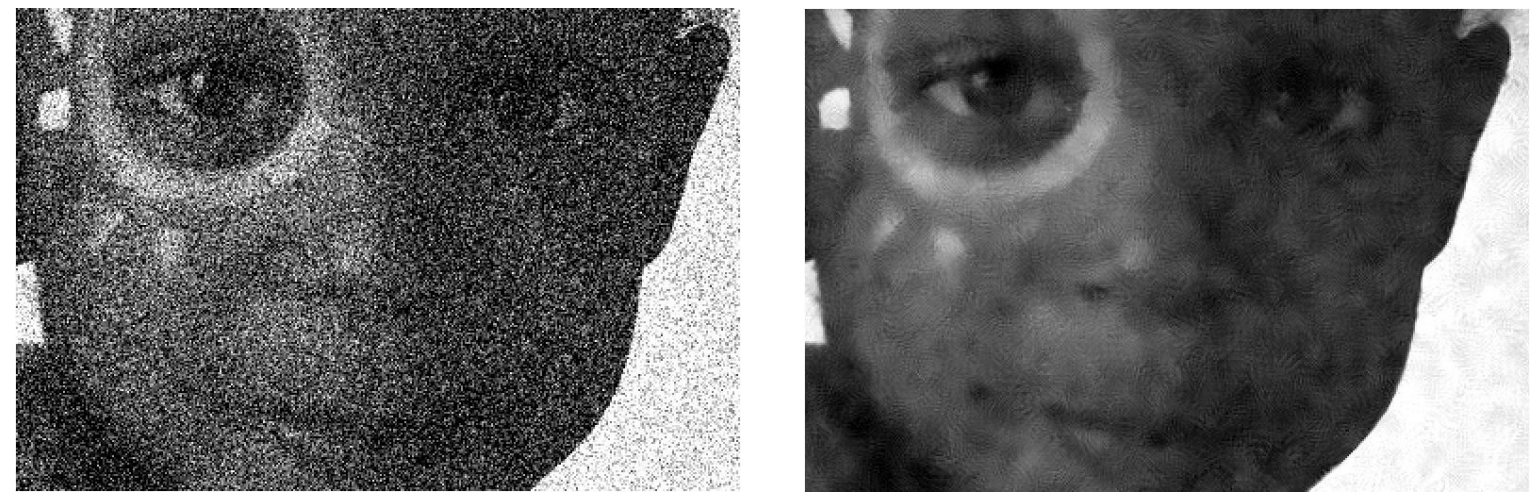

(b) Noise

(c) NLM
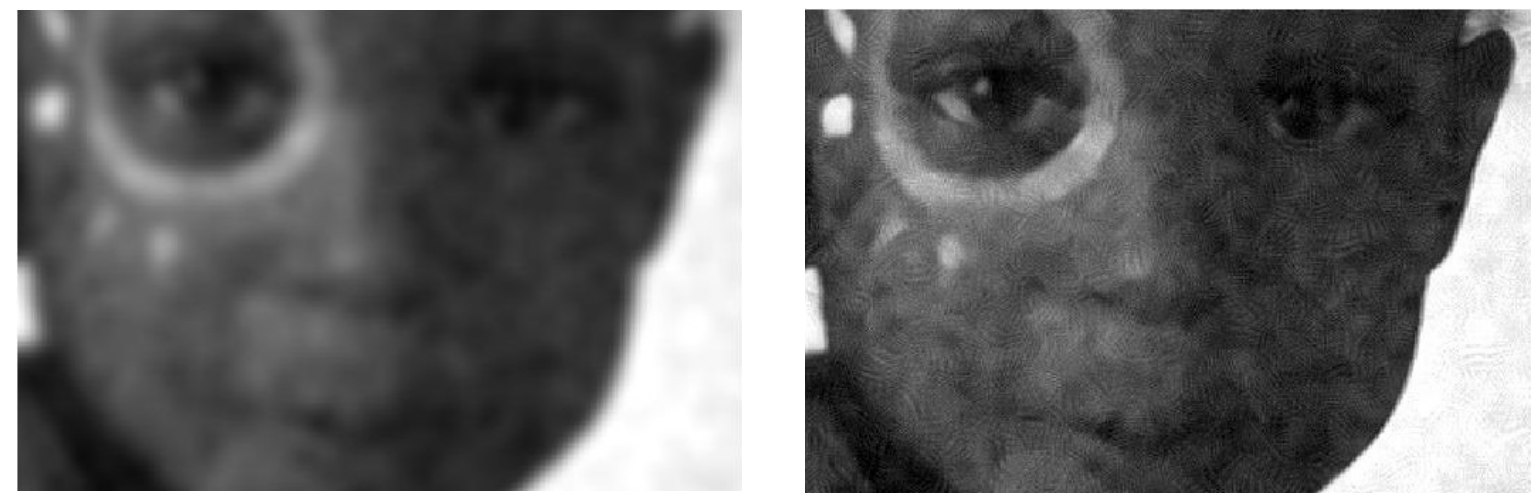

(d) TS-NLM

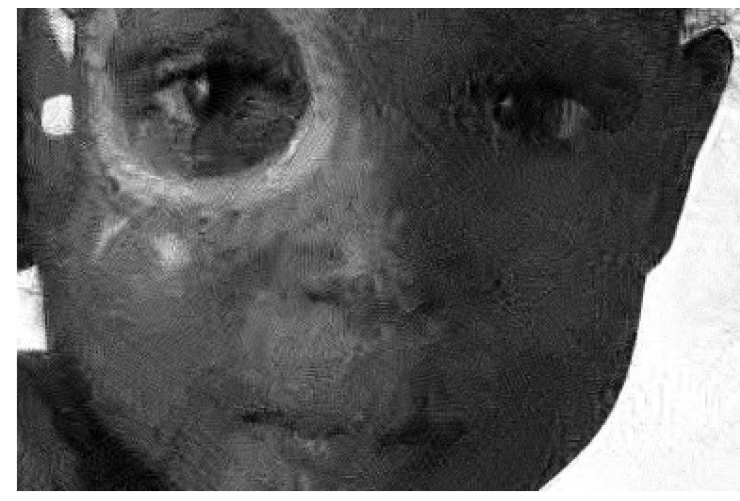

(e) NLEM

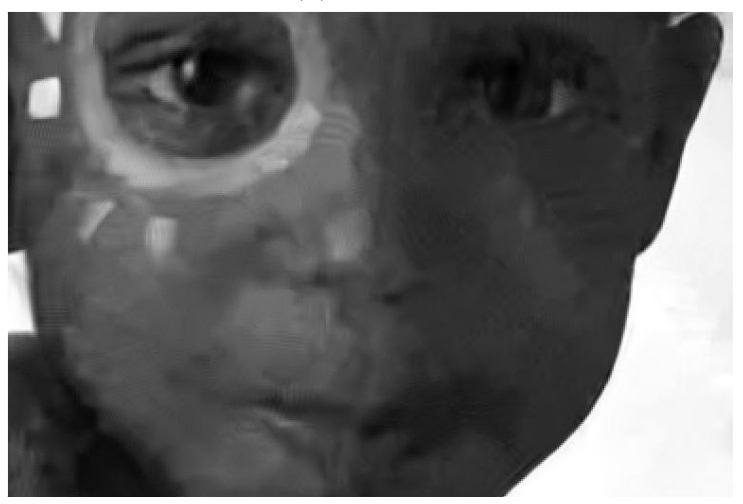

(g) Proposed

(f) BM3D

Figure 4.12: Visual Comparison (Zoom) for $\sigma=60$ 


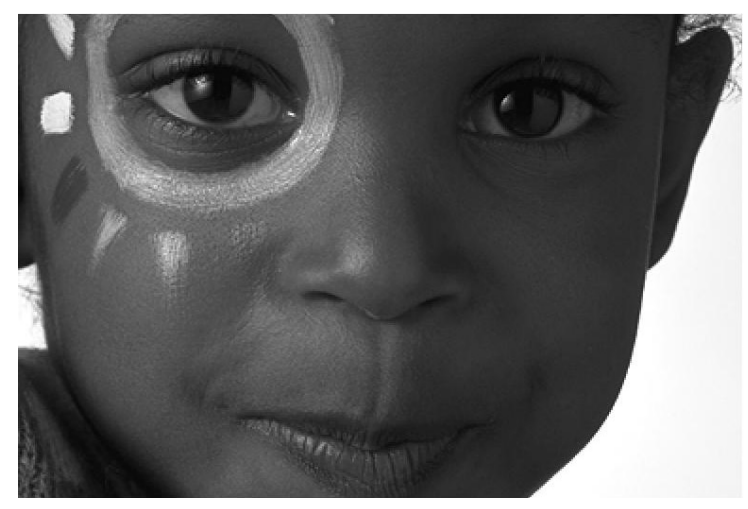

(a) Original

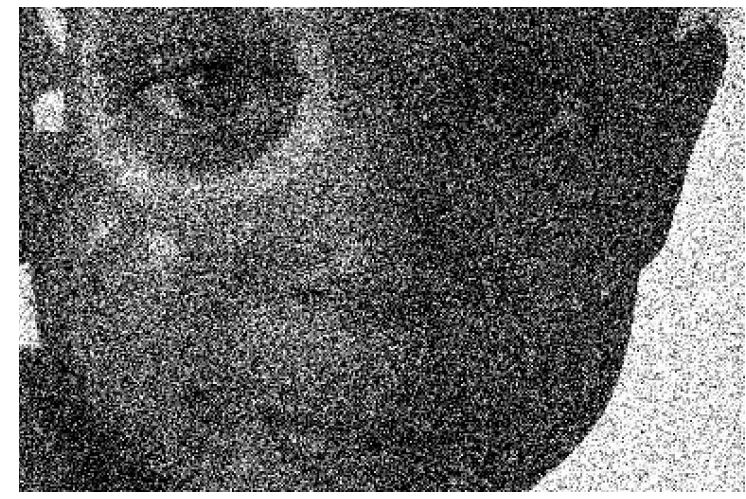

(b) Noise

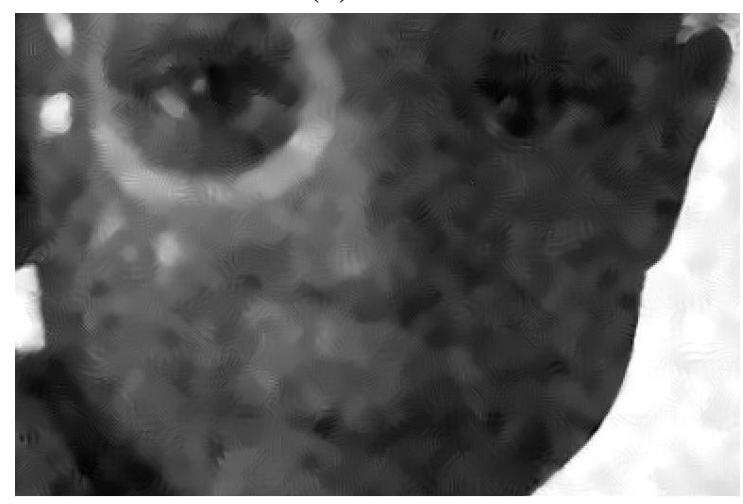

(d) TS-NLM

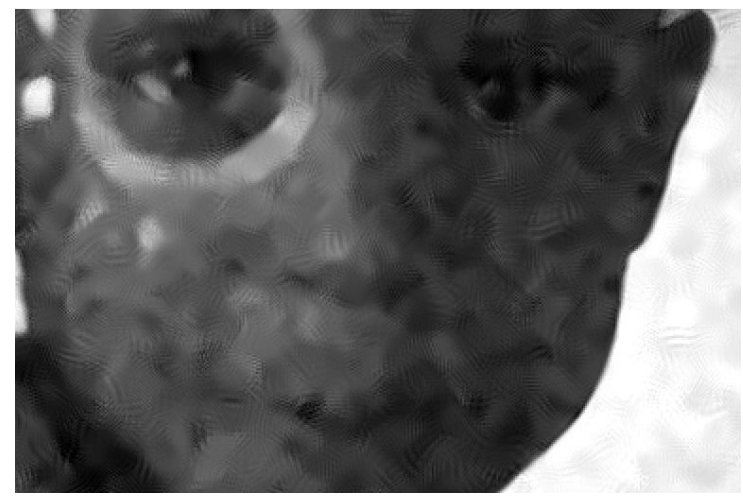

(f) BM3D

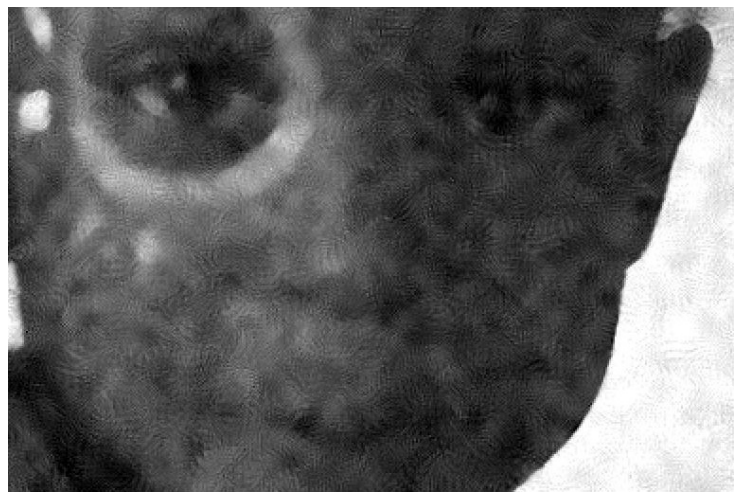

(c) NLM

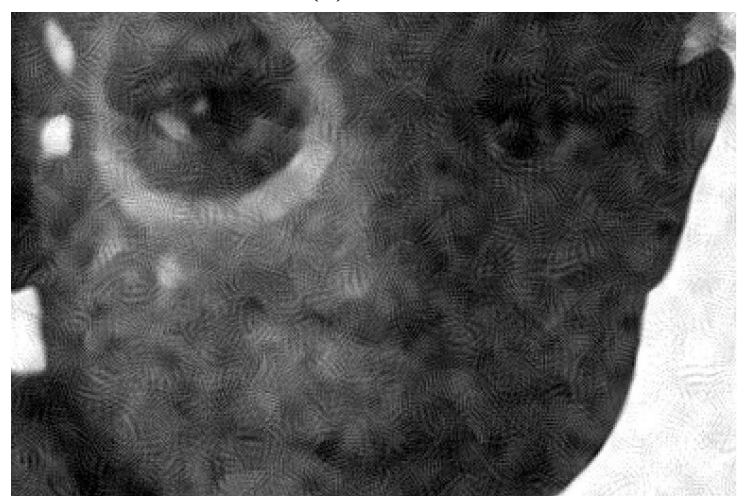

(e) NLEM

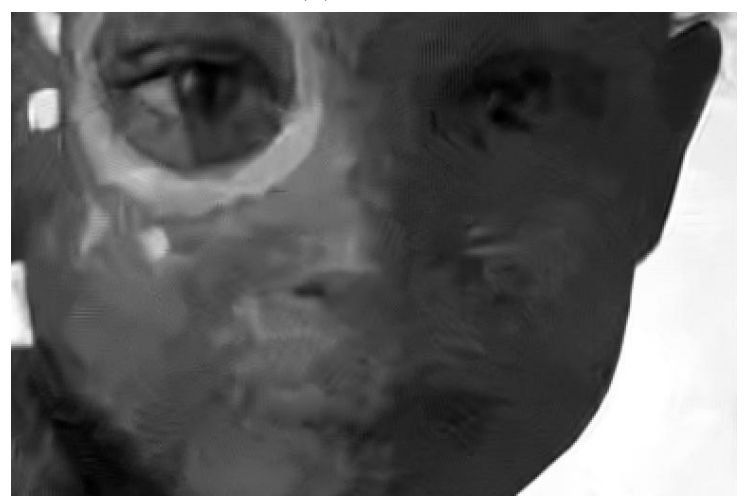

(g) Proposed

Figure 4.13: Visual Comparison (Zoom) for $\sigma=80$ 


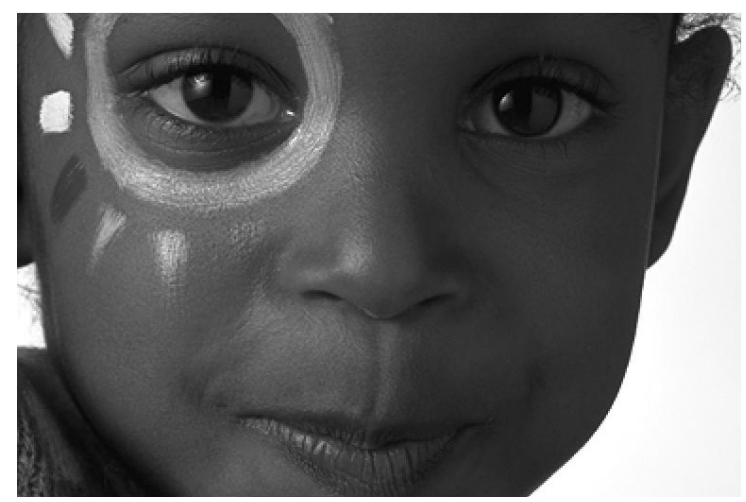

(a) Original

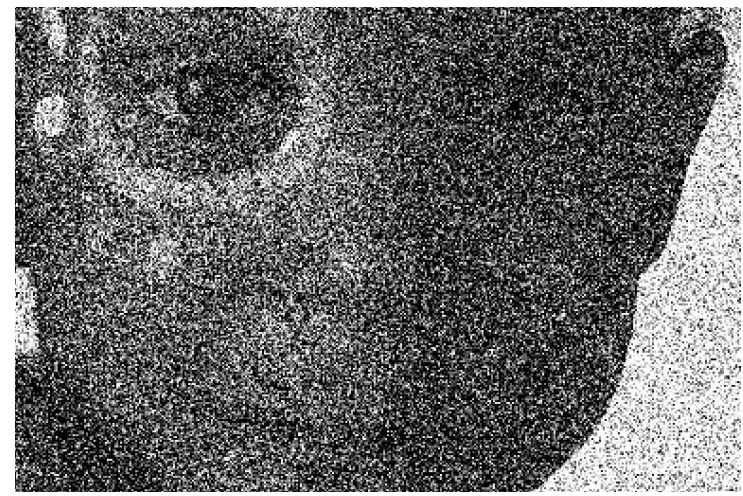

(b) Noise

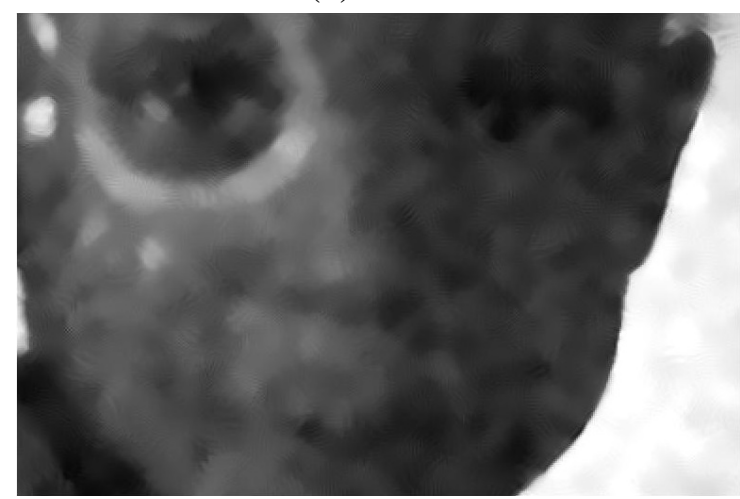

(d) TS-NLM

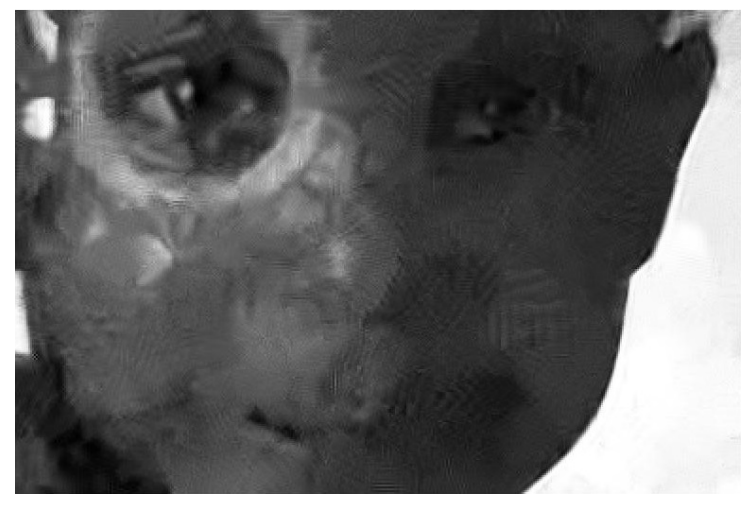

(f) $\mathrm{BM} 3 \mathrm{D}$

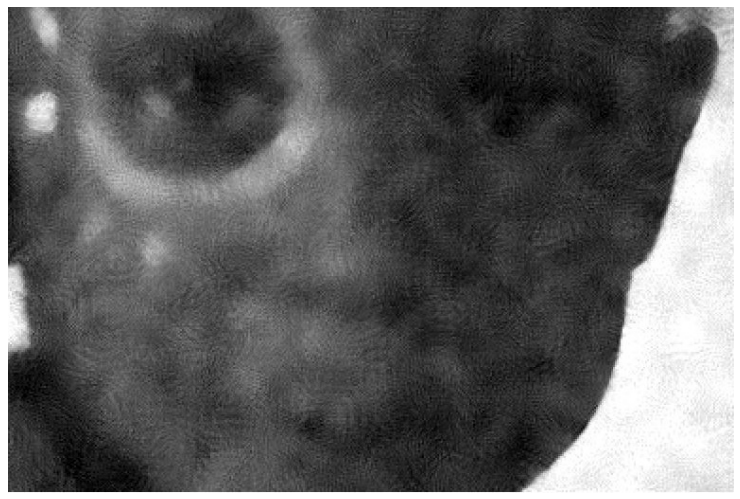

(c) NLM

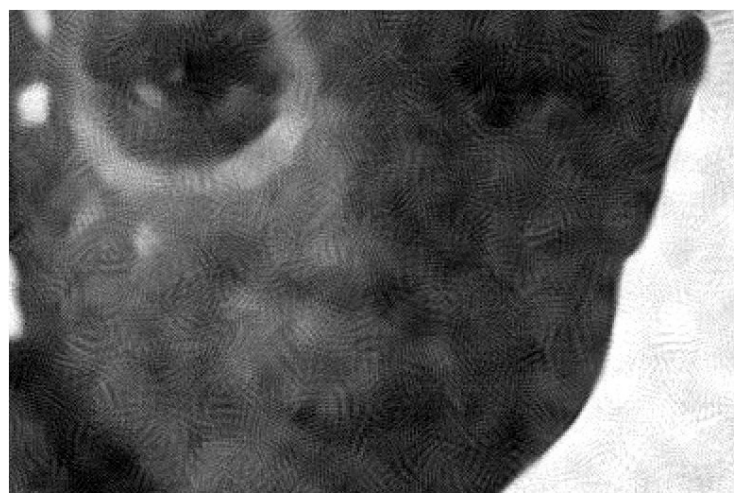

(e) NLEM

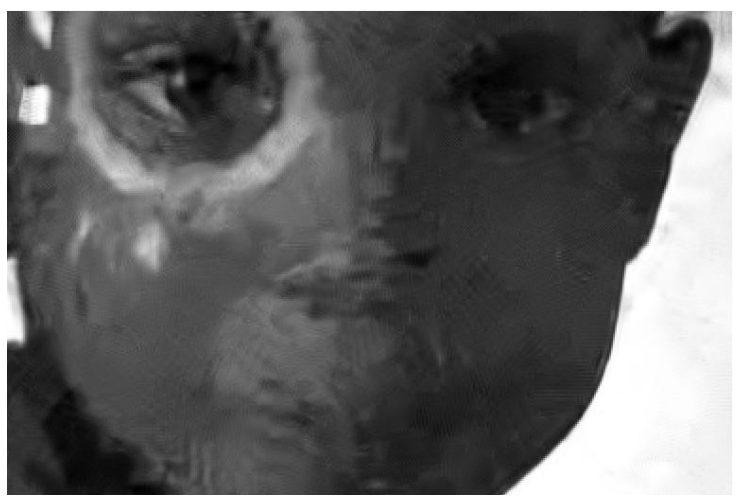

(g) Proposed

Figure 4.14: Visual Comparison (Zoom) for $\sigma=100$ 


\subsubsection{Intensity Profile}

The image intensity profile can help analyze how similar the profile of a denoised image is to that of the original noise-free image. Figure 4.15 shows the chosen horizontal scan line 100 from the Girl image. Figure 4.16 and Figure 4.17 show the intensity profile for the true image and the noisy image at noise level 40 and 80, respectively. The Pearson correlation coefficient between the original intensity profile and the profile of the noisy and each of the denoised images is shown in Table 4.7 (for $\sigma=40$ ) and Table 4.8 (for $\sigma=80$ ).

The intensity profile of the proposed method shows better preservation of edges and textures, represented as sharp changes in profile graph. Non-local means and its variants attenuates the local peaks in the intensity profile, representing more blurring in the denoised image. For noise level $\sigma=80$, the original non-local means method and its variants have more noise artifacts remaining, as represented by the more jagged lines in the section of the profile graph, closer to the origin. The proposed method has the closest resemblance to the intensity profile of the original image. When comparing the Pearson correlation coefficient, the correlation between the intensity profile of the original image and the proposed method is higher compared to those of the other existing methods. 
Table 4.7: Pearson correlation coefficient comparison of the proposed method, the noisy image, the NLM method, variants of NLM and BM3D denoising scheme for noise $\sigma=40$

\begin{tabular}{|c|c|c|c|c|c|}
\hline Noise Image & NLM & TS-NLM & NLEM & BM3D & Proposed \\
\hline 0.890 & 0.980 & 0.981 & 0.985 & 0.990 & 0.993 \\
\hline
\end{tabular}

Table 4.8: Pearson correlation coefficient comparison of the proposed method, the noisy image, the NLM method, variants of NLM and BM3D denoising scheme for noise $\sigma=80$

\begin{tabular}{|c|c|c|c|c|c|}
\hline Noise Image & NLM & TS-NLM & NLEM & BM3D & Proposed \\
\hline 0.680 & 0.973 & 0.977 & 0.978 & 0.987 & 0.988 \\
\hline
\end{tabular}

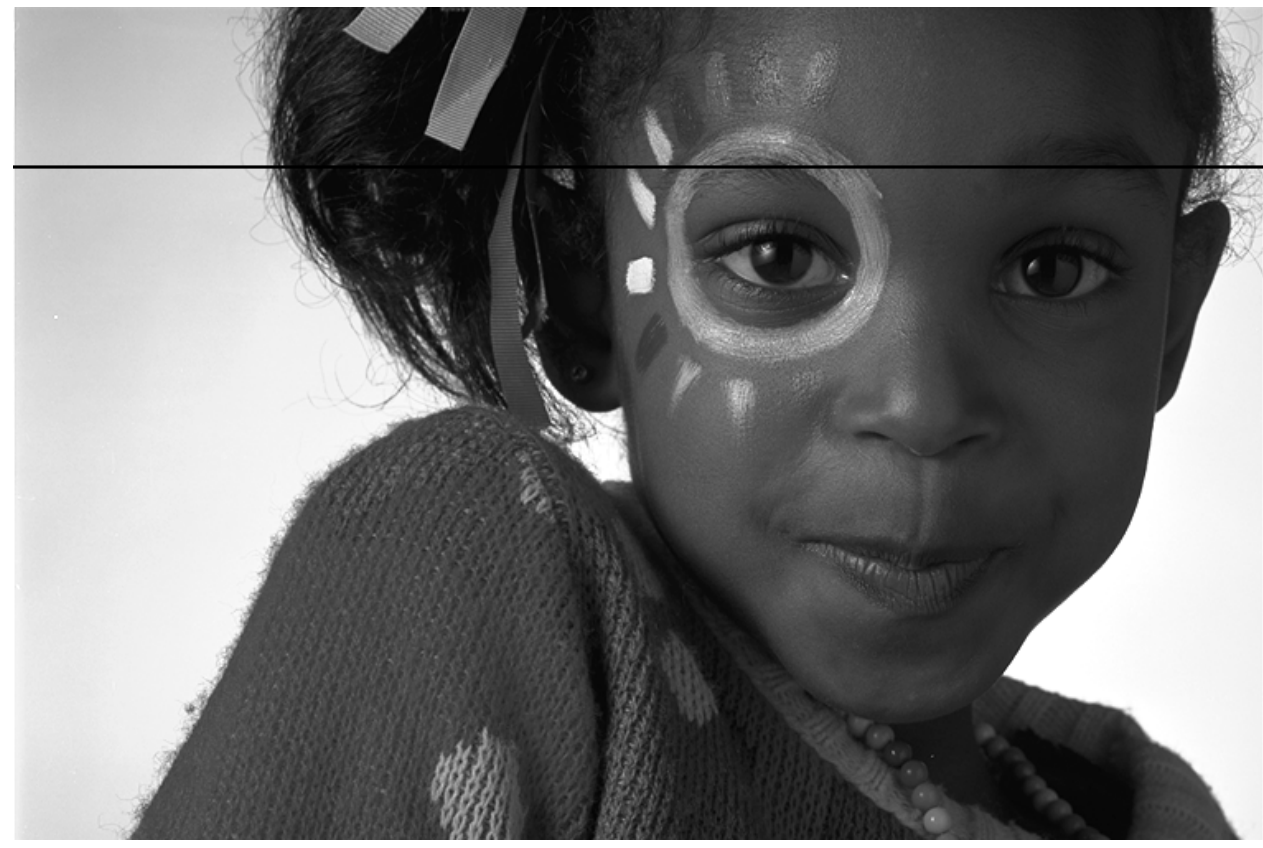

Figure 4.15: Row number 100 of Girl image used for generating intensity profile. Scan line shown as a red line. 


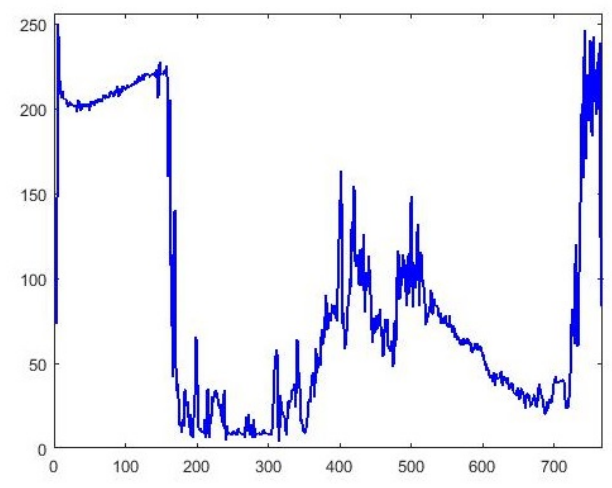

(a) Original

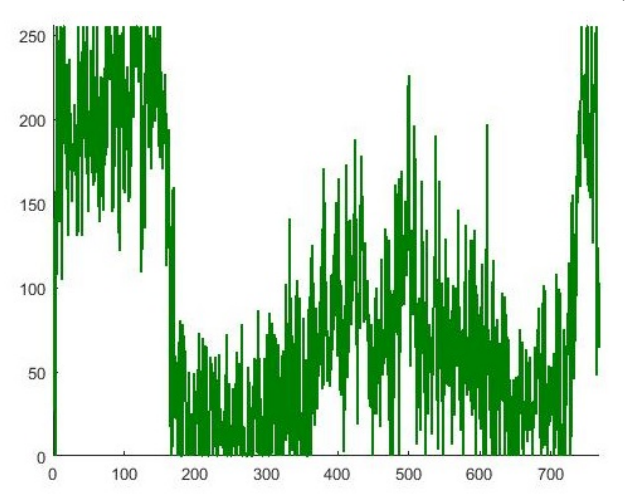

(b) Noise

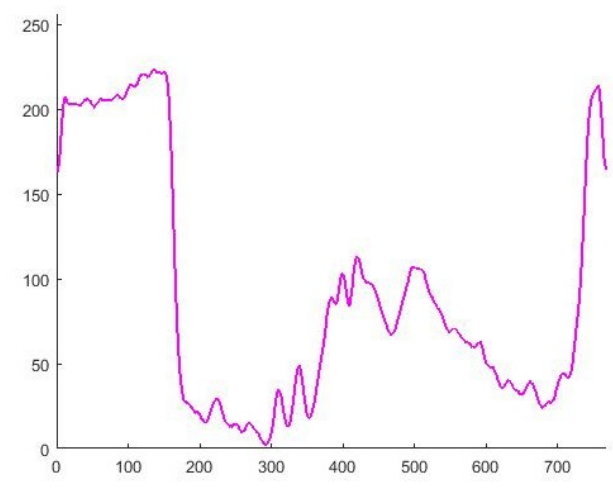

(d) TS-NLM

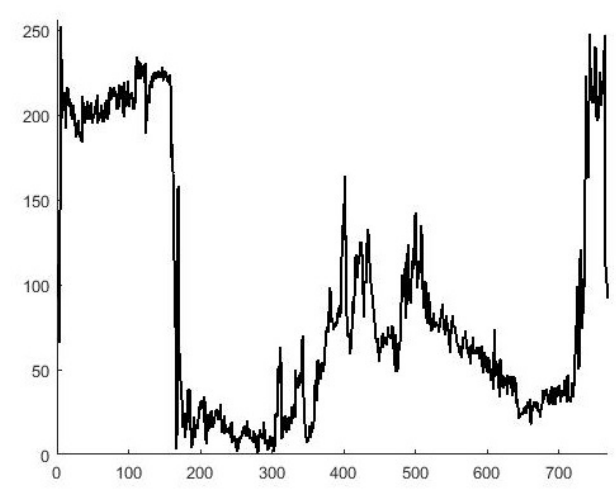

(f) BM3D

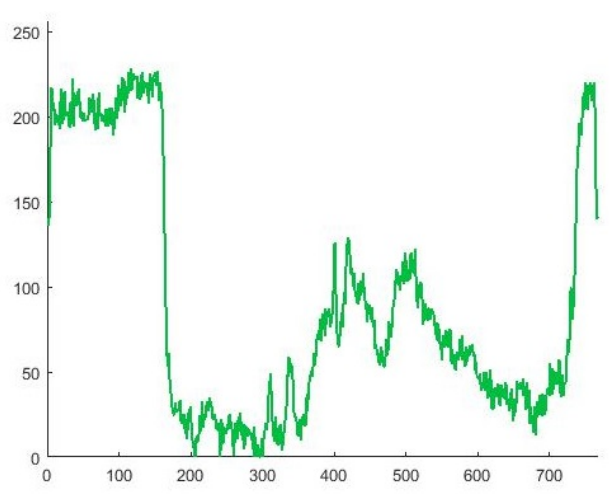

(c) NLM

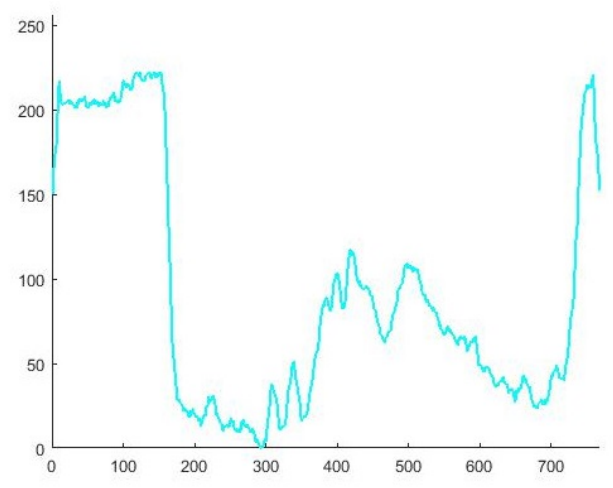

(e) NLEM

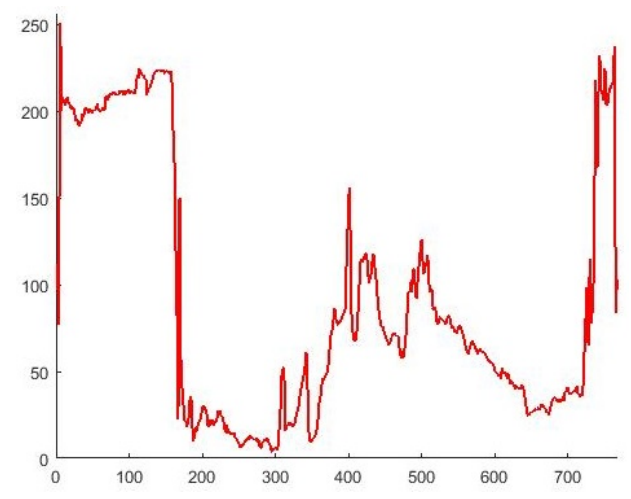

(g) Proposed

Figure 4.16: Intensity Profile Comparison of the Girl image at scan line $100(\sigma=40)$ 


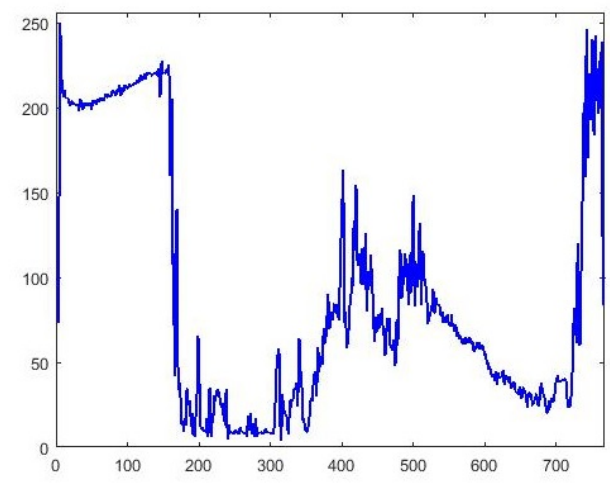

(a) Original

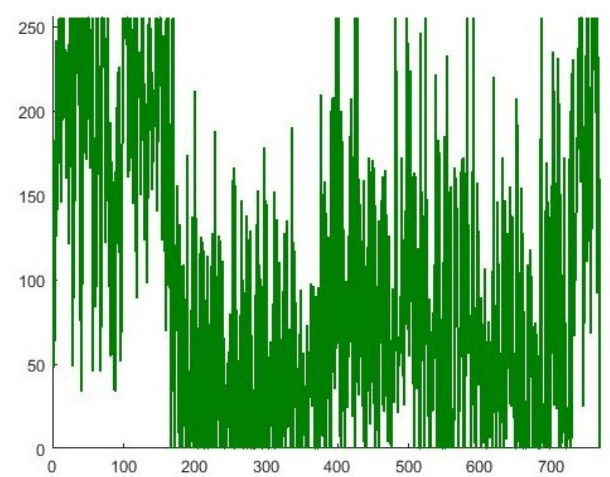

(b) Noise

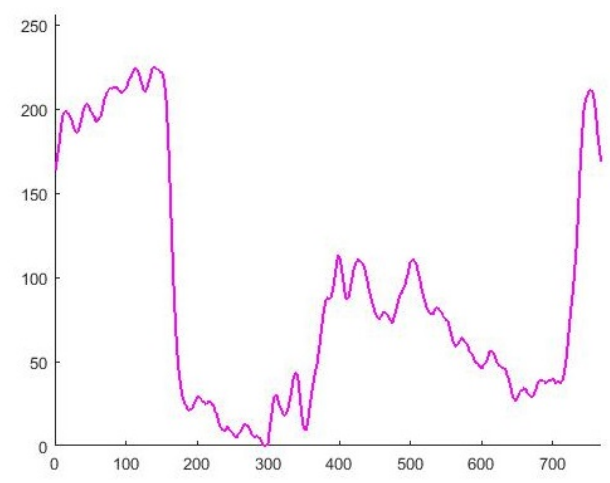

(d) TS-NLM

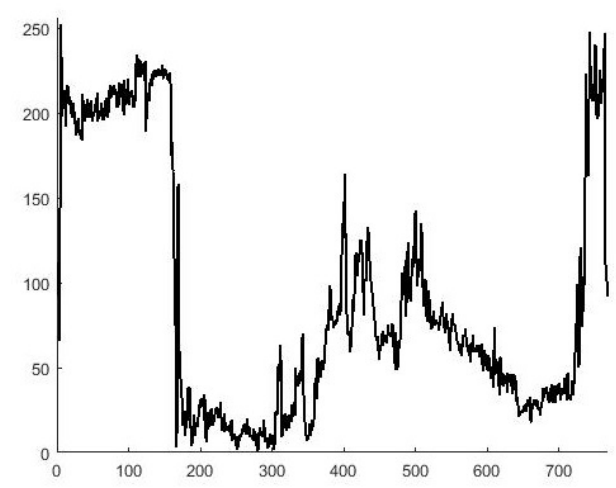

(f) BM3D

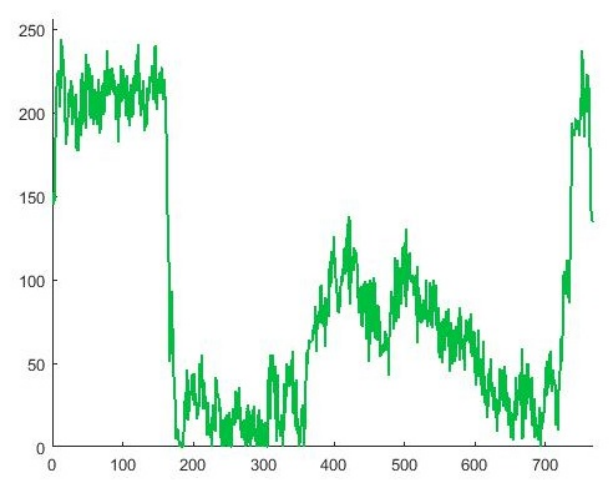

(c) NLM

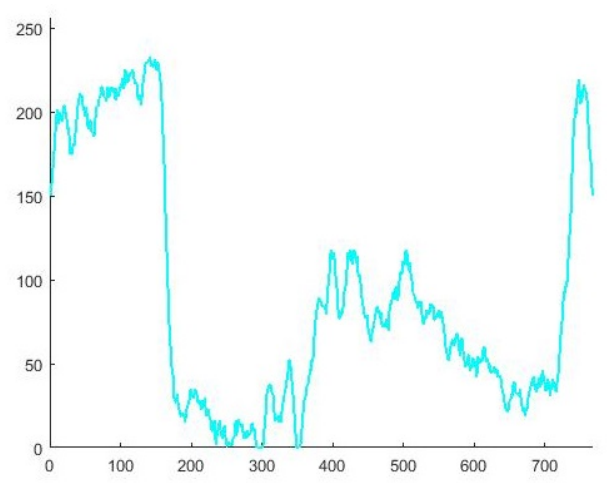

(e) NLEM

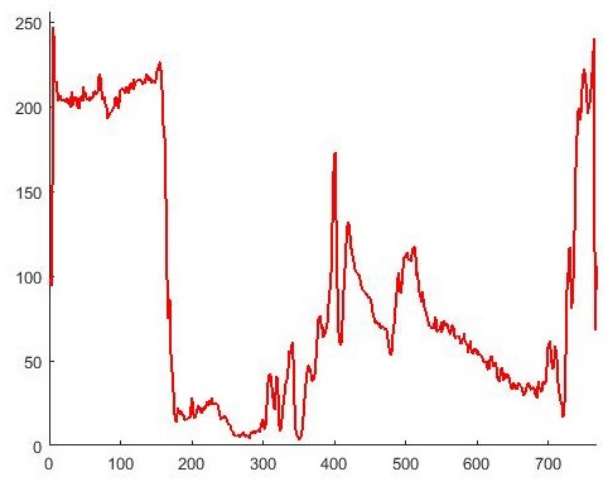

(g) Proposed

Figure 4.17: Intensity Profile Comparison of the Girl image at scan line $100(\sigma=80)$ 


\subsection{Summary}

The objective comparisons have shown that our proposed method has been able to achieve better denoising performance, in terms of PSNR and MSSIM, compared to the original non-local means scheme as well as its variants. When compared to BM3D, another state-of-the-art method for image denoising, our proposed method has managed better results only for low noise levels $(\sigma \leq 80)$.

Visual quality comparison of the denoised image has shown that the denoised image produced by our proposed method has been able to maintain more texture and details when compared to the original non-local means method and its variants. The noise artifacts in the denoised image are better attenuated in our proposed method, the difference been significantly noticeable at high noise levels. 


\section{Chapter 5}

\section{Conclusion and Future Work}

\subsection{Conclusion}

In this thesis, we have proposed an improved variant of the original non-local means scheme, the patch-based approach for denoising additive Gaussian noise in the spatial domain. The proposed method thresholds the weights of the pixels defined around a search area of the pixel being denoised. The thresholded weights are used for weighted averaging, whereby pixels below a defined cutoff weight are ignored. The cut-off weight is determined based on the noise level estimation of an image.

For a noise level, the patch and search window size are determined by a model, which is empirically defined through a learning approach. The proposed method is applied in a two-step approach for image denoising. The proposed method has demonstrated better objective and subjective denoising performance, compared to the original non-local means algorithm and its variants. 
When compared to $\mathrm{BM} 3 \mathrm{D}$, the state-of-the-art approach for image denoising, the proposed method demonstrated better results when $\sigma \leq 80$.

\subsection{Future Work}

Our improved variant of the non-local means method has been proposed for denoising additive Gaussian noise in grayscale images. The proposed method can be considered to extend to color image denoising. Instead of using the raw pixel gray values, the luminance and chrominance information can be used for estimating denoised pixels.

The proposed method can also be extended for video image application. For video applications, denoising a pixel will depend of a reference frame at time $t$ and use the similar patch information from previous $t-1$ frames to estimate the denoised pixels. This will result in more pixels being included in the denoising process. 


\section{Bibliography}

[1] S. Aja-Fernandez, G. Vegas-Snchez-Ferrero, M. Martn-Fernndez, and C. Alberola-Lpez. Automatic noise estimation in images using local statistics. additive and multiplicative cases. Image and Vision Computing, 27(6):750-770, 2009.

[2] T. Bronx and D. Cremers. Iterated non-local means for texture restoration. In Proc. Scale Space and Variational Methods in Computer Vision, 2007.

[3] A. Buades, B. Coll, and J. Morel. A non-local algorithm for image denoising. In Proc. Computer Vision and Pattern Recognition, 2005.

[4] A. Buades, B. Coll, and J. Morel. A review of image denoising algorithms, with a new one. SIAM Journal on Multiscale Modeling and Simulation: A SIAM Interdisciplinary Journal, 4:490-530, 2005.

[5] K.N. Chaudhury and A. Singer. Non-Local Euclidean Medians. IEEE Signal Processing Letters, 19(11):745-748, 2012. 
[6] K. Dabov, A. Foi, V. Katkovnik, and K. Egiazarian. Image denoising by sparse 3D transform-domain collaborative filtering. IEEE Transactions on Image Processing, 16(8):2080 - 2095, 2007.

[7] K. Dabov, A. Foi, V. Katkovnik, and K. Egiazarian. BM3D image denoising with shape-adaptive principal component analysis. In Proc. Signal Processing with Adaptive Sparse Structured Representation, 2009.

[8] V. Frost, J. Stiles, K. Shanmugan, and J. Holtzman. A model for radar images and its application to adaptive digital filtering of multiplicative noise. IEEE Transactions on Pattern Analysis Machine Intelligence, 4(2):157$166,1982$.

[9] R.C. Gonzalez and R.E. Woods. Digital Image Processing. Prentice-Hall Inc, 2008.

[10] Y. Han and R. Chen. Effective video denoising based on dynamic nonlocal means. Image and Vision Computing, 30(2):78-85, 2012.

[11] M. Hasan and M. El-Sakka. Structural similarity optimized wiener filter: A way to fight image noise. In Proc. International Conference on Image Analysis and Recognition, 2014.

[12] S. Hu and W. Hou. Denosing 3D ultrasound images by non-local means accelerated by GPU. In Proc. International Conference on Intelligent Computation and Bio-Medical Instrumentation, 2011. 
[13] M. Iftikhar, S. Rathore, A. Jalil, and M. Hussain. A novel extension to non-local means algorithm: Application to brain mri de-noising. In Proc. International Multi Topic Conference, 2013.

[14] F. Jin, P. Fieguth, L. Winger, and E. Jernigan. Adaptive Wiener filtering of noisy images and image sequences. In Proc. IEEE Int. Conf. Image Process, 2003.

[15] Z. Kelm, D. Blezek, B. Bartholmai, and B. Erickson. Optimizing nonlocal means for denoising low dose CT. In Proc. International Symposium on Biomedical Imaging: From Nano to Macro, 2009.

[16] J. Koenderink. The structure of images. In Proc. Biological Cybernatics, 1984.

[17] D. Kuan, A. Sawchuk, T. Strand, and P. Chavel. Adaptive noise smoothing filter for images with signal-dependent noise. IEEE Transaction on Pattern Analysis and Machine Intelligence, 7(2):165-177, 1985.

[18] J. Lee. Digital image enhancement and noise filtering by using local statistics. IEEE Transactions on Pattern Analysis Machine Intelligence, 2(2):165-168, 1980.

[19] S.J. Lim. Two-Dimensional Signal and Image Processing. Prentice Hall, New Jersey, USA, 1990. 
[20] G.M. Maruf and M.R. El-Sakka. Improved non-local means algorithm based on dimensionality reduction. In Proc. International Conference on Image Analysis and Recognition, 2015.

[21] A. Mouton, N. Megherbi, G. T. Flitton, S. Bizot, and T. P. Breckon. Gaussian noise estimation in digital images using nonlinear sharpening and genetic optimization. In Proc. Instrumentation and Measurement Technology Conference, 2007.

[22] A. Mouton, N. Megherbi, G. T. Flitton, S. Bizot, and T. P. Breckon. A novel intensity limiting approach to metal artefact reduction in $3 \mathrm{~d}$ ct baggage imagery. In Proc. International Conference on Image Processing, 2012.

[23] P. Perona and J. Malik. Scale-space and edge detection using anisotropic diffusion. IEEE Transactions on Pattern Analysis and Machine Intelligence, 12(7):629-639, 1990.

[24] A. Rehman and Z. Wang. SSIM-based non-local means image denoising. In Proc. Image Processing, 2011.

[25] F. Russo. Noise estimation in digital images using fuzzy processing. In Proc. International Conference on Image Processing, 2001.

[26] T. Tasdizen. Principal components for non-local means image denoising. In Proc. International Conference on Image Processing, 2008. 
[27] Z. Wang and A. Bovik. A universal image quality index. IEEE Signal Processing Letters, 9(3):81-84, 2002.

[28] Z. Wang, E. Simoncelli, and A. Bovik. Multiscale structural similarity for image quality assessment. In Proc. Thirty-Seventh Asilomar Conference on Signals, Systems and Computers, 2004.

[29] A. Witkin. Scale-space filtering: A new approach to multi-scale description. In Proc. IEEE International Conference on Acoustics, Speech, and Signal Processing, 1984.

[30] Q. Xu, H. Jiang, R. Scopigno, and M. Sbert. A new approach for very dark video denoising and enhancement. In Proc. International Conference on Image Processing, 2010.

[31] L. Zhang, W. Dong, D. Zhang, and G. Shi. Two-stage image denoising by principal component analysis with local pixel grouping. Pattern Recognition, 43(4):1531-1549, 2010.

[32] Y. Zhao and Y. Liu. Patch based saliency detection method for 3d surface simplification. In Proc. International Conference on Pattern Recognition, 2012.

[33] S. Zhu, Y. Li, and Y. Li. Two-stage non-local means filtering with adaptive smoothing parameter. International Journal for Light and Electron Optics, 125(23):7040-7044, 2014. 


\title{
Curriculum Vitae
}

\author{
Name: $\quad$ Asif Khan
}

Education and Islamic University of Technology

Degrees: 2009 - 2012 B.Sc.

University of Western Ontario

London, ON

2014 - 2015 M.Sc.

Honours and Western Graduate Research Scholarship (WGRS)

Awards: 2014-2015

Related Work Teaching Assistant

Experience: The University of Western Ontario

2014 - 2015

\section{Publications:}

A. Khan and M. El-Sakka, "Non-Local Means using Adaptive Weight Thresholding", International Conference on Computer Vision Theory and Applications, 2016 (Accepted)

A. Khan, "Adaptive Non-Local Means using Weight Thresholding", Three Minute Thesis presentation at Fallona Family Interdisciplinary Showcase, 2015 